\title{
A new equilibrated residual method improving accuracy and efficiency of flux-free error estimates
}

\author{
N. Parés, P. Díez \\ Laboratori de Càlcul Numèric (LaCàN) - Universitat Politècnica de Catalunya \\ Campus Nord UPC, E-08034 Barcelona, Spain
}

\begin{abstract}
This paper presents a new methodology to compute guaranteed upper bounds for the energy norm of the error in the context of linear finite element approximations of the reaction-diffusion equation. The new approach revisits the ideas in $[20,18]$ with the goal of substantially reducing the computational cost of the flux-free method while retaining the good quality of the bounds. The new methodology provides also a technique to compute equilibrated boundary tractions improving the quality of standard equilibration strategies. The zeroth-order equilibration conditions are imposed using an alternative less restrictive form of the first-order equilibration conditions, along with a new efficient minimization criterion. This new equilibration strategy provides much more accurate upper bounds for the energy and requires only doubling the dimension of the local linear systems of equations to be solved.
\end{abstract}

Keywords: exact/guaranteed/strict bounds, fully computable a posteriori error estimation, adaptivity, reaction-diffusion equation, flux-free, equilibrated boundary tractions

\section{Introduction}

The certification of numerical simulations is fundamental in any engineering design process. In particular, most simulations are aimed at obtaining a certified approximation of a certain quantity of interest $[9,5]$. This paper focuses in obtaining bounds for the error in energy norm, which is an essential ingredient for computing bounds for any quantity of interest. Specifically, for the advection-reaction-diffusion equation, upper and lower bounds for the error in a given quantity of interest are obtained from upper bounds for the energy norm of the error of symmetrized auxiliary reaction-diffusion problems $[25,18,19]$.

The two implicit residual a posteriori error estimates providing computable guaranteed bounds for the energy norm (and also for quantities of interest through an error representation involving an adjoint problem) are: (1) the hybrid-flux techniques which require computing equilibrated tractions around the elements, and (2) the flux-free techniques where the local problems are defined in patches of elements around every vertex node of the mesh and no explicit computation of equilibrated tractions is required. A comparison of the two techniques is presented in $[20,18]$ showing that the flux-free approach provides much more accurate results while having larger computational cost.

The objective of the present work is to provide a novel flux-free strategy that while retaining the accuracy of the standard flux-free approach, its computational cost, which is the main drawback of flux-free error estimates, is comparable to the cost of hybrid-flux techniques.

The remainder of the paper is organized as follows. The model problem and the flux-free a posteriori error estimate are presented in Sections 2 to 5. Section 6 reformulates the new flux-free a posteriori error estimate into a new hybrid-flux technique allowing to obtain accurate equilibrated tractions. The paper concludes with a brief computational cost overview in Section 7 and several numerical examples in Section 8. 


\section{Model problem and finite element approximation}

Let $\Omega$ be an open bounded polygonal domain in $\mathbb{R}^{2}$, with boundary $\partial \Omega=\bar{\Gamma}_{\mathrm{N}} \cup \bar{\Gamma}_{\mathrm{D}}$, where $\Gamma_{\mathrm{N}}$ and $\Gamma_{\mathrm{D}}$ form a disjoint partition of the boundary. The boundary value problem to be solved is stated as follows: find the real-valued function $u$ such that

$$
\begin{aligned}
-\Delta u+\kappa^{2} u & =f & & \text { in } \Omega, \\
u & =u_{\mathrm{D}} & & \text { on } \Gamma_{\mathrm{D}}, \\
\nabla u \cdot \boldsymbol{n} & =g_{\mathrm{N}} & & \text { on } \Gamma_{\mathrm{N}},
\end{aligned}
$$

where $u_{\mathrm{D}}$ is assumed to be continuous and piecewise linear on the Dirichlet boundary $\Gamma_{\mathrm{D}}$. Additionally, without loss of generality $\kappa$ is assumed to be a non-negative constant and in order to guarantee a unique solution of $(1)$, either $\kappa>0$ or $\Gamma_{\mathrm{D}}$ is a non-empty set.

The standard variational formulation of the problem consists of seeking $u \in \mathcal{U}$ with

$$
a(u, v)=\ell(v) \quad \text { for all } v \in \mathcal{V}
$$

where

$$
a(u, v)=\int_{\Omega}\left(\nabla u \cdot \nabla v+\kappa^{2} u v\right) d \Omega \quad \text { and } \quad \ell(v)=\int_{\Omega} f v d \Omega+\int_{\Gamma_{\mathrm{N}}} g_{\mathrm{N}} v d \Gamma .
$$

The solution and test spaces are $\mathcal{U}=\left\{u \in \mathcal{H}^{1}(\Omega),\left.u\right|_{\Gamma_{\mathrm{D}}}=u_{\mathrm{D}}\right\}$ and $\mathcal{V}=\left\{v \in \mathcal{H}^{1}(\Omega),\left.v\right|_{\Gamma_{\mathrm{D}}}=0\right\}$, $\mathcal{H}^{1}(\Omega)$ being the standard Sobolev space of functions defined in $\Omega$ such that both the functions and their first derivatives are square-integrable.

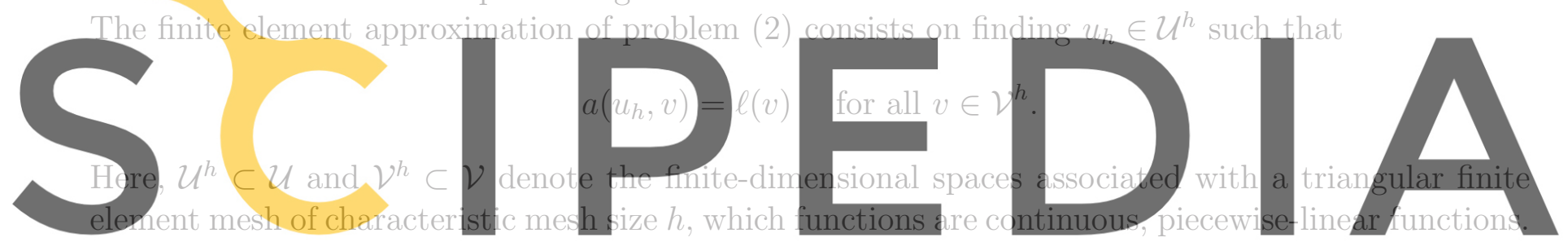

The mesh is the union of non-overlapping linear triangular elements, denoted by $\Omega_{k}, k=1, \ldots, n_{\mathrm{el}}$,

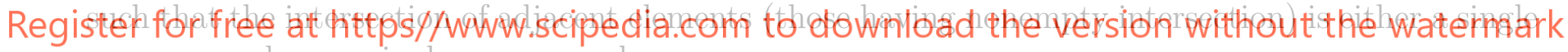
common node or a single common edge.

\section{Guaranteed upper bounds for the energy norm: Complementary energy relaxation}

Much effort has been devoted to obtain strict bounds, that is, bounds guaranteed with respect to the exact solution independently of any underlying mesh (see for instance the series of references [24, 25, $14,27,16,17,7,20,18,19]$ ). All these strategies recover strict bounds of the error (measured either using the energy norm or using a particular quantity of interest) using the standard complementary energy approach. The key idea is to relax the continuous residual error problem of finding $e=$ $u-u_{h} \in \mathcal{V}$ such that

$$
a(e, v)=\ell(v)-a\left(u_{h}, v\right) \quad \forall v \in \mathcal{V}
$$

by introducing dual unknowns living in larger spaces with less regularity requirements.

The relaxed problem consists in obtaining a pair of dual estimates $\boldsymbol{q} \in\left[\mathcal{L}^{2}(\Omega)\right]^{2}$ and $r \in \mathcal{L}^{2}(\Omega)$ such that

$$
\int_{\Omega}\left(\boldsymbol{q} \cdot \boldsymbol{\nabla} v+\kappa^{2} r v\right) d \Omega=\ell(v)-a\left(u_{h}, v\right) \quad \forall v \in \mathcal{V} .
$$

Any pair of dual estimates $\boldsymbol{q}$ and $r$ verifying equation (4) yield an upper bound for the energy norm of the error $\left\|\left|\|\mid\|\right.\right.$, where the energy norm of a function $v$ is $\|v\|=a(v, v)^{1 / 2}$. Indeed, joining equations 
(3) and (4) and using the Cauchy-Schwarz inequality yields

$$
\begin{aligned}
a(e, v) & =\int_{\Omega}\left(\boldsymbol{q} \cdot \boldsymbol{\nabla} v+\kappa^{2} r v\right) d \Omega \\
& \leq\left(\int_{\Omega}\left(\boldsymbol{q} \cdot \boldsymbol{q}+\kappa^{2} r^{2}\right) d \Omega\right)^{1 / 2}\left(\int_{\Omega}\left(\boldsymbol{\nabla} v \cdot \nabla v+\kappa^{2} v^{2}\right) d \Omega\right)^{1 / 2} \\
& =\left(\|\boldsymbol{q}\|_{\left[\mathcal{L}^{2}(\Omega)\right]^{2}}^{2}+\kappa^{2}\|r\|_{\mathcal{L}^{2}(\Omega)}^{2}\right)^{1 / 2}\|v\| \quad \forall v \in \mathcal{V} .
\end{aligned}
$$

Thus, substituting $v=e$ in the previous equation and noting that $\|e\|^{2}=a(e, e)$ yields the guaranteed upper bound

$$
\|e\|^{2} \leq\|\boldsymbol{q}\|^{2}+\kappa^{2}\|r\|^{2}
$$

where, to ease the notation, the subscript of the previous $\mathcal{L}^{2}$-norms is omitted.

Remark 1. Note that the relaxed problem (4) admits at least a trivial solution $\boldsymbol{q}=\boldsymbol{\nabla} e \in\left[\mathcal{L}^{2}(\Omega)\right]^{2}$ and $r=e \in \mathcal{L}^{2}(\Omega)$. In this case, expression (5) turns into an equality. Any other dual estimates $q$ and $r$ fulfilling (4) yield upper bounds for $\|\mid\| \|$. However, in order to obtain sharper values for the upper bound, the combined norm of $q$ and $r$ should be the least possible.

\section{Data projection and data oscillation errors}

The methods providing strict or guaranteed upper bounds from (5) are based in obtaining fully
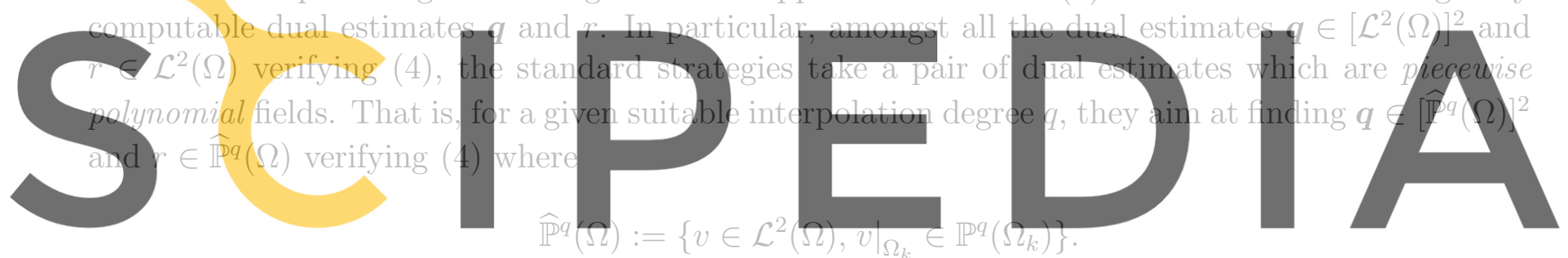

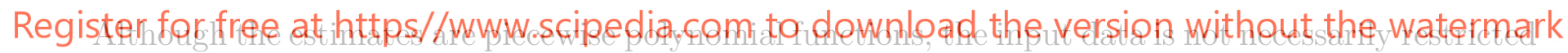

to being of the same type. This restriction, present in some previous references, can be precluded by isolating the data oscillation errors from those associated with the discretization, as shown below. Denote by $\Pi_{k}^{\hat{q}}: \mathcal{L}^{2}\left(\Omega_{k}\right) \rightarrow \mathbb{P}^{\hat{q}}\left(\Omega_{k}\right)$ the $\mathcal{L}^{2}\left(\Omega_{k}\right)$-orthogonal projector to the space of polynomials of degree $\hat{q}$ defined over the element $\Omega_{k}$, and by $\Pi_{\gamma}^{\bar{q}}: \mathcal{L}^{2}(\gamma) \rightarrow \mathbb{P}^{\bar{q}}(\gamma)$ the $\mathcal{L}^{2}(\gamma)$-orthogonal projector to the space of polynomials of degree $\bar{q}$ defined over the edge $\gamma$. Then, for $\hat{q} \geq 1$ and $\bar{q} \geq 1$, it holds that

$$
\int_{\Omega_{k}} f v d \Omega=\int_{\Omega_{k}} \Pi_{k}^{\hat{q}} f v d \Omega \quad \text { for all } v \in \mathbb{P}^{1}\left(\Omega_{k}\right)
$$

and

$$
\int_{\gamma} g_{\mathrm{N}} v d \Gamma=\int_{\gamma} \Pi_{\gamma}^{\bar{q}} g_{\mathrm{N}} v d \Gamma \quad \text { for all } v \in \mathbb{P}^{1}(\gamma), \gamma \subset \Gamma_{\mathrm{N}}
$$

The following theorem shows that guaranteed upper bounds for the error can be obtained separating the data oscillation errors from the discretization errors. The proof of this Theorem is included in Appendix A.

Theorem 1. Let $\boldsymbol{q} \in\left[\mathcal{L}^{2}(\Omega)\right]^{2}$ and $r \in \mathcal{L}^{2}(\Omega)$ be a pair of dual estimates verifying

$$
\int_{\Omega}\left(\boldsymbol{q} \cdot \nabla v+\kappa^{2} r v\right) d \Omega=\ell^{\Pi}(v)-a\left(u_{h}, v\right) \quad \forall v \in \mathcal{V}
$$


for

$$
\ell^{\Pi}(v)=\sum_{i=1}^{n_{\mathrm{el}}}\left[\int_{\Omega_{k}} \Pi_{k}^{\hat{q}} f v d \Omega+\sum_{\gamma \subset \Gamma_{\mathrm{N}} \cap \partial \Omega_{k}} \int_{\gamma} \Pi_{\gamma}^{\bar{q}} g_{\mathrm{N}} v d \Gamma\right],
$$

where $\hat{q}$ and $\bar{q}$ are two non-negative natural numbers.

Then, the following upper bound follows

$$
\|e\|^{2} \leq \sum_{k=1}^{n_{\mathrm{el}}} \eta_{k}^{2}
$$

where

$$
\eta_{k}=\sqrt{\|\boldsymbol{q}\|_{\left[\mathcal{L}^{2}\left(\Omega_{k}\right)\right]^{2}}^{2}+\kappa^{2}\|r\|_{\mathcal{L}^{2}\left(\Omega_{k}\right)}^{2}}+\operatorname{osc}_{k}(f)+\sum_{\gamma \subset \Gamma_{\mathrm{N}} \cap \partial \Omega_{k}}{ }_{\operatorname{sic}}\left(g_{\gamma}\left(g_{\mathrm{N}}\right) .\right.
$$

The oscillation terms are given by

$$
\operatorname{osc}_{k}(f)=C_{0}\left\|f-\prod_{k}^{\hat{q}} f\right\|_{\mathcal{L}^{2}\left(\Omega_{k}\right)}
$$

$$
\operatorname{osc}_{\gamma}\left(g_{\mathrm{N}}\right)=\min \left\{C_{1}, C_{2}\right\}\left\|g_{\mathrm{N}}-\Pi_{\gamma}^{\bar{q}} g_{\mathrm{N}}\right\|_{\mathcal{L}^{2}(\gamma)},
$$

and the exactly computable data oscillation constants are
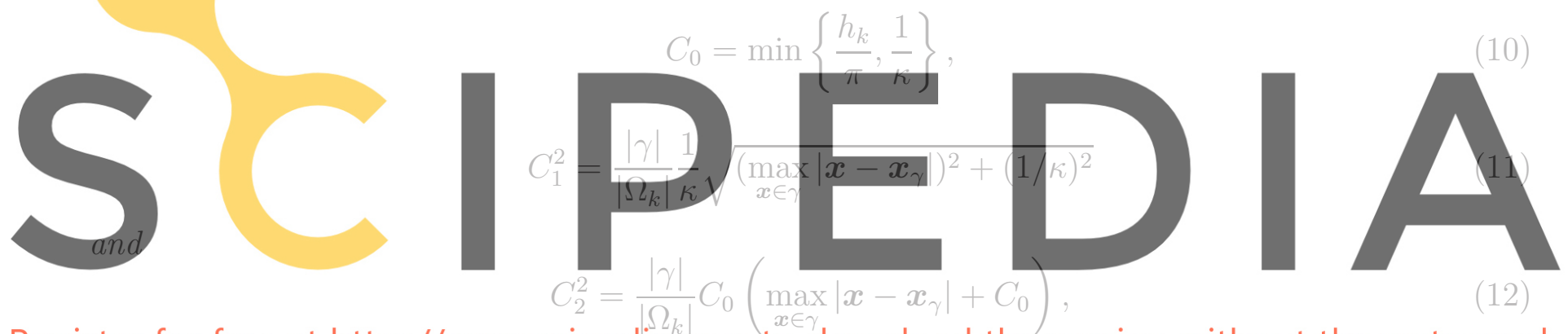

(12)

Register for free at https//www.scipedia.com to download the version without the watermark $x_{\gamma}$ being the vertex of element $\Omega_{k}$ opposite to the edge $\gamma,|\gamma|$ being the length of the edge $\gamma$ and $h_{k}$ and $\left|\Omega_{k}\right|$ being the diameter and area of element $\Omega_{k}$ respectively.

Remark 2. Note that in the expressions for $C_{1}$ and $C_{2}$, the term $\max _{\boldsymbol{x} \in \gamma}\left|\boldsymbol{x}-\boldsymbol{x}_{\gamma}\right|$ can be replaced by $h_{k}$ if desired and the bounds still hold.

Remark 3. In the case $\kappa=0$, the oscillation errors are given by

$$
\operatorname{osc}_{k}(f)=C_{0}\left\|f-\Pi_{k}^{\hat{q}} f\right\|_{\mathcal{L}^{2}\left(\Omega_{k}\right)}
$$

and

$$
\operatorname{osc}_{\gamma}\left(g_{\mathrm{N}}\right)=C_{2}\left\|g_{\mathrm{N}}-\Pi_{\gamma}^{\bar{q}} g_{\mathrm{N}}\right\|_{\mathcal{L}^{2}(\gamma)},
$$

where the constants are simplified to

$$
C_{0}=\frac{h_{k}}{\pi}
$$

and

$$
C_{2}^{2}=\frac{|\gamma|}{\left|\Omega_{k}\right|} \frac{h_{k}}{\pi}\left(\max _{\boldsymbol{x} \in \gamma}\left|\boldsymbol{x}-\boldsymbol{x}_{\gamma}\right|+\frac{h_{k}}{\pi}\right) .
$$

Hereafter, to ease the notation, the subscript of the $\mathcal{L}^{2}$-norms is simplified, and the following notations are used $\|\cdot\|_{\left[\mathcal{L}^{2}\left(\Omega_{k}\right)\right]^{2}}=\|\cdot\|_{k},\|\cdot\|_{\mathcal{L}^{2}\left(\Omega_{k}\right)}=\|\cdot\|_{k}$ and $\|\cdot\|_{\mathcal{L}^{2}(\gamma)}=\|\cdot\|_{\gamma}$. Note that the same notation is used for the local norm of vector and scalar fields in $\Omega_{k}$, since they can be clearly distinguished by their arguments. 


\section{Local computation of the dual estimates $q$ and $r$ using a flux-free approach: a novel cheaper construction}

This section is devoted to introduce a new computational strategy for the piecewise polynomial dual estimates. Following [20, 18], equation (8) is imposed in a subdomain-based approach. The computational cost is drastically reduced using a novel methodology based in an explicit/closed expression for the dual estimates.

The description of the novel approach is organized as follows: first, the domain decomposition technique allowing to compute upper bounds for $\|e \mid\|$ solving local constrained optimization problems is presented, followed by the introduction of its equivalent strong form (Sections 5.1 and 5.2). Section 5.3 provides a closed expression for the dual estimates verifying the local constraints of the optimization problems. This expression allows reducing the constrained optimization problems into unconstrained quadratic optimization problems that are described in detail in Section 5.4.

\subsection{Domain decomposition: local optimization problems and basic notations}

For each particular vertex node $\boldsymbol{x}_{i}$, let $\omega_{i}$ be the support of its associated linear shape function $\phi_{i}$, also referred to as the patch of elements connected to node $i$ of the mesh or the star associated with node $i$. Let also $\mathcal{V}\left(\omega_{i}\right)$ and $\widehat{\mathbb{P}}^{q}\left(\omega_{i}\right)$ denote the local restrictions of the spaces $\mathcal{V}$ and $\widehat{\mathbb{P}}^{q}(\Omega)$ to the star $\omega_{i}$. Then, for each star, we aim at computing $q^{i} \in\left[\widehat{\mathbb{P}}^{3}\left(\omega_{i}\right)\right]^{2}$ and $r^{i} \in \widehat{\mathbb{P}}^{3}\left(\omega_{i}\right)$ minimizing

\section{$\left\|q^{i}\right\|_{\left[\mathcal{L}^{2}\left(\omega_{i}\right)\right]^{2}}^{2}+\kappa^{2}\left\|r^{i}\right\|_{\mathcal{L}^{2}\left(\omega_{i}\right)}^{2}$}

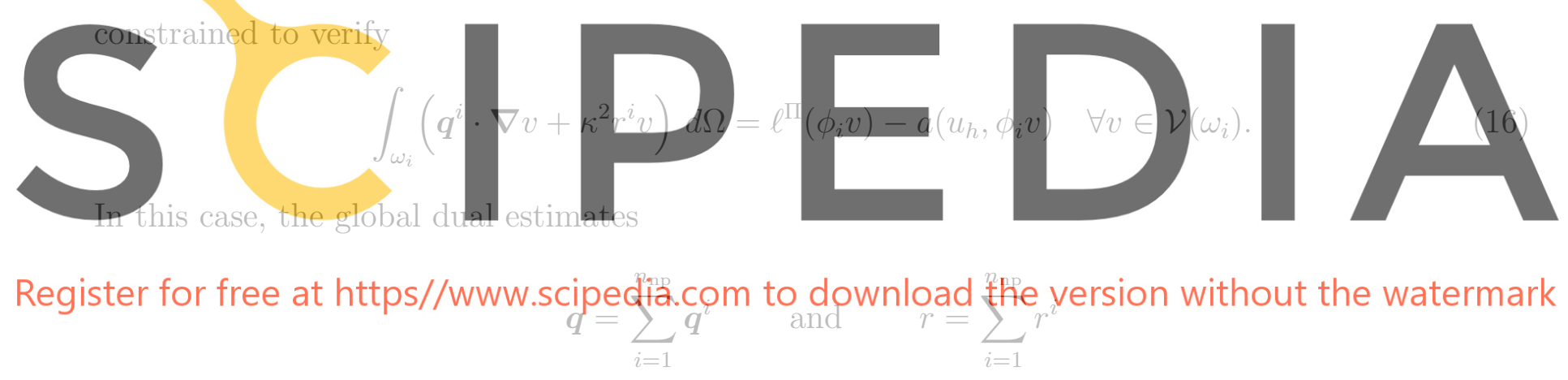

verify equation (8). Indeed, adding the local star equations (16) and using the partition of unity property of the linear shape functions, $\sum_{i=1}^{n_{\mathrm{np}}} \phi_{i}=1$, yields the desired result. Moreover, the minimization of the local norm given in (15) yields bounds with very good quality.

In order to restrict the dual estimates to be cubic piecewise polynomial $(q=3)$, the polynomial degrees for the projected data $\ell^{\Pi}(\cdot)$ are $\hat{q}=1$ and $\bar{q}=0$. That is, a piecewise linear interpolation is used for the interior data $f$ whereas a piecewise constant interpolation for the Neumann tractions $g_{\mathrm{N}}$ is used.

\subsection{Strong form of the constraints of the local optimization problems}

The computation of the piecewise polynomial dual estimates starts considering a strong version of (16). For a given star $\omega_{i}$, we distinguish two disjoint sets of edges: those where the basis function $\phi_{i}$ vanishes, that is

$$
\mathcal{Z}_{i}=\left\{\gamma \subset \omega_{i} \text { such that }\left.\phi_{i}\right|_{\gamma}=0\right\}
$$

and the interior edges of the star (or edges radiating from node $\boldsymbol{x}_{i}$ )

$$
\Gamma_{i}=\left\{\gamma \subset \omega_{i} \text { such that } \phi_{i}(\boldsymbol{x}) \neq 0 \text { for some } \boldsymbol{x} \in \gamma\right\} .
$$

Note that for any star $\omega_{i}, \Gamma_{i}$ coincides with the edges connecting node $i$ with the rest of the nodes of the star. If the star is associated to an interior node, $\mathcal{Z}_{i}$ coincides with $\partial \omega_{i}$. 
Also, for each edge $\gamma$, let $\boldsymbol{n}^{\gamma}$ be an arbitrary but fixed unit normal (one amongst the two possible). If $\gamma$ is an exterior edges, $\boldsymbol{n}^{\gamma}$ coincides with the outward unit normal to $\partial \Omega, \boldsymbol{n}$. Let $g_{\gamma}^{i}$ denote the external loading traction associated to the star $\omega_{i}$ and the each edge $\gamma$ (in the direction of $\boldsymbol{n}^{\gamma}$ ) such that

$$
\begin{array}{lr}
g_{\gamma}^{i}=0 & \text { on } \gamma \subset \mathcal{Z}_{i}, \\
g_{\gamma}^{i}=\phi_{i} \Pi_{\gamma}^{0} g_{\mathrm{N}} & \text { on } \gamma \subset \Gamma_{i} \cap \Gamma_{\mathrm{N}} .
\end{array}
$$

Then, a strong version of the local error equation (16) can be written as: for all the elements in the star $\Omega_{k} \subset \omega_{i}$ find $\boldsymbol{q}_{k}^{i} \in\left[\mathbb{P}^{3}\left(\Omega_{k}\right)\right]^{2}$ and $r_{k}^{i} \in \mathbb{P}^{3}\left(\Omega_{k}\right)$ such that

$$
\begin{aligned}
-\boldsymbol{\nabla} \cdot\left(\boldsymbol{q}_{k}^{i}+\phi_{i} \boldsymbol{\nabla} u_{h}\right)+\kappa^{2} r_{k}^{i} & =\phi_{i}\left(\Pi_{k}^{1} f-\kappa^{2} u_{h}\right)-\boldsymbol{\nabla} u_{h} \cdot \boldsymbol{\nabla} \phi_{i} & & \text { in } \Omega_{k} \\
\left(\boldsymbol{q}_{k}^{i}+\phi_{i} \boldsymbol{\nabla} u_{h}\right) \cdot \boldsymbol{n}_{k}^{\gamma} & =\sigma_{k}^{\gamma} g_{\gamma}^{i} & & \text { on } \gamma \subset \partial \Omega_{k},
\end{aligned}
$$

where given an element $\Omega_{k}$ and an edge of this element $\gamma \subset \partial \Omega_{k}, \boldsymbol{n}_{k}^{\gamma}$ denotes the outward unit normal to the element associated with edge $\gamma$ and $\sigma_{k}^{\gamma}=\boldsymbol{n}^{\gamma} \cdot \boldsymbol{n}_{k}^{\gamma}$. The following notation $\boldsymbol{q}_{k}^{i}=\left.\boldsymbol{q}^{i}\right|_{\Omega}$ and $r_{k}^{i}=r^{i} \Omega_{\Omega}$ has been used to denote the restriction of the dual estimates to element $\Omega_{k} \in \omega_{i}$. Appendix B shows that indeed for a given set of tractions $\left\{g_{\gamma}^{i}\right\}_{\gamma \subset \omega_{i}}$ verifying (17) then any pair of dual estimates fulfilling (18) are also a solution of (16).

An important point of the new approach presented here, is that we restrict the local tractions $g^{i}$ to be linear at the edges of the star. Thus, it is possible finding $q_{k}^{i} \in\left[\mathbb{P}^{3}\left(\Omega_{k}\right)\right]^{2}$ and $r_{k}^{i} \in \mathbb{P}^{3}\left(\Omega_{k}\right)$

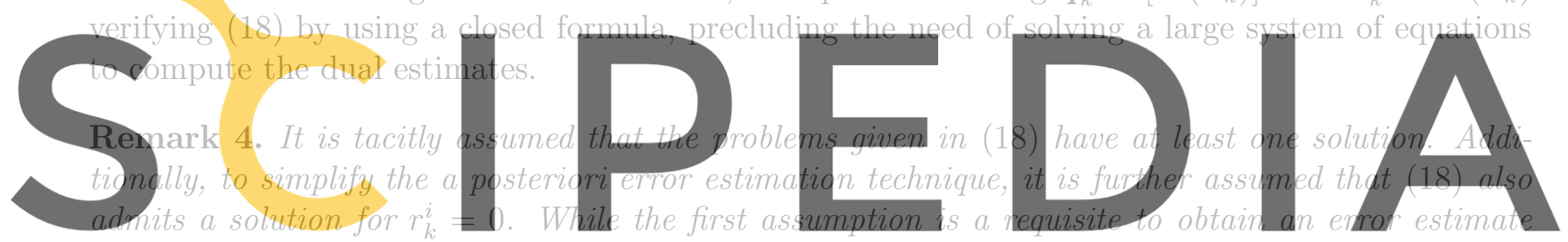

using a flux-free approach, the second assumption is only introduced for a simpler presentation, and

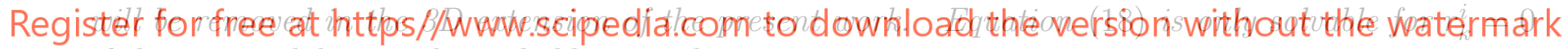

if the compatibility condition holds, namely

$$
\ell^{\Pi}\left(\phi_{i}\right)-a\left(u_{h}, \phi_{i}\right)=0
$$

For $\hat{q} \geq 1$ and $\bar{q} \geq 1$, this holds and is easily shown using the definition of the projections (6) and (7) from which $\ell^{\Pi}\left(\phi_{i}\right)=\ell\left(\phi_{i}\right)$ along with the Galerkin orthogonality given by taking $v=\phi_{i}$ in (2). Note however, that equation (18) is associated to $\hat{q}=1$ and $\bar{q}=0$, therefore some solvability issues may appear in a star intersecting the Neumann boundary. For the sake of a simpler presentation, it is assumed that either all the boundary conditions are of Dirichlet type or $g_{\mathrm{N}}$ is piecewise constant in $\Gamma_{\mathrm{N}}$ (in which case $\Pi_{\gamma}^{1} g_{\mathrm{N}}=\Pi_{\gamma}^{0} g_{\mathrm{N}}$ ). Alternatively, one can let $\bar{q}=1$ for which $\phi_{i} \Pi_{\gamma}^{1} g_{\mathrm{N}} \in \mathbb{P}^{2}(\gamma)$ and the approach given in Appendix E should be used to consider quadratic (instead of linear) tractions in the Neumann boundaries.

\subsection{Closed expression for the dual estimates verifying the constraints of the optimization problems}

In order to introduce the closed formula for the dual estimates, some notation has to be introduced. Let $\Omega_{k}$ be a given triangle in the star $\omega_{i}$ defined by the vertices $\boldsymbol{x}_{[1]}, \boldsymbol{x}_{[2]}$ and $\boldsymbol{x}_{[3]}$ with associated opposite edges $\gamma_{[1]}, \gamma_{[2]}$ and $\gamma_{[3]}$ of length $l_{[1]}=\left|\gamma_{[1]}\right|, l_{[2]}=\left|\gamma_{[2]}\right|$ and $l_{[3]}=\left|\gamma_{[3]}\right|$ respectively, as shown in Figure 1, where subscripts within brackets, like in $\boldsymbol{x}_{[1]}$, refer to local numbering. Denote also by $\boldsymbol{t}_{[1]}=\boldsymbol{x}_{[3]}-\boldsymbol{x}_{[2]}, \boldsymbol{t}_{[2]}=\boldsymbol{x}_{[1]}-\boldsymbol{x}_{[3]}$ and $\boldsymbol{t}_{[3]}=\boldsymbol{x}_{[2]}-\boldsymbol{x}_{[1]}$ the vectors describing the edges, and by $\boldsymbol{n}_{[1]}, \boldsymbol{n}_{[2]}$ and $\boldsymbol{n}_{[3]}$ the unit outward normal vectors to edges $\gamma_{[1]}, \gamma_{[2]}$ and $\gamma_{[3]}$ respectively. Note that with this notation $\boldsymbol{n}_{[i]}=\boldsymbol{n}_{k}^{\gamma_{[i]}}$. Finally, let $\lambda_{[1]}, \lambda_{[2]}$ and $\lambda_{[3]}$ be the barycentric coordinates of the triangle (which inside the triangle coincide with the linear shape functions associated to the nodes $\boldsymbol{x}_{[1]}, \boldsymbol{x}_{[2]}$ and $\boldsymbol{x}_{[3]}$ 


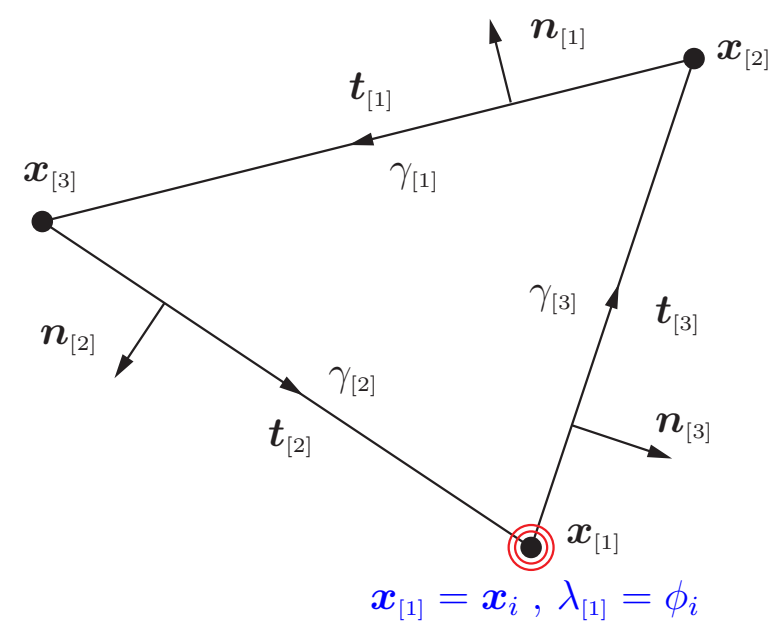

Figure 1: Notation for the vertices and edges of an element contained in star $\omega_{i}$.

respectively). Let also $\mathcal{N}=\left\{1,2, \ldots, n_{\mathrm{np}}\right\}$ denote the set of indices of the nodes of the finite element mesh, and let $\mathcal{N}(\gamma) \subset \mathcal{N}$ and $\mathcal{N}\left(\Omega_{k}\right) \subset \mathcal{N}$ denote the set of indices of the nodes of edge $\gamma$ and element $\Omega_{k}$ respectively.

The approximate tractions in the edges, which are linear functions in the edges, are then defined using the coefficients $\left\{\alpha_{\gamma i}^{m}, m \in \mathcal{N}(\gamma)\right\}$ as

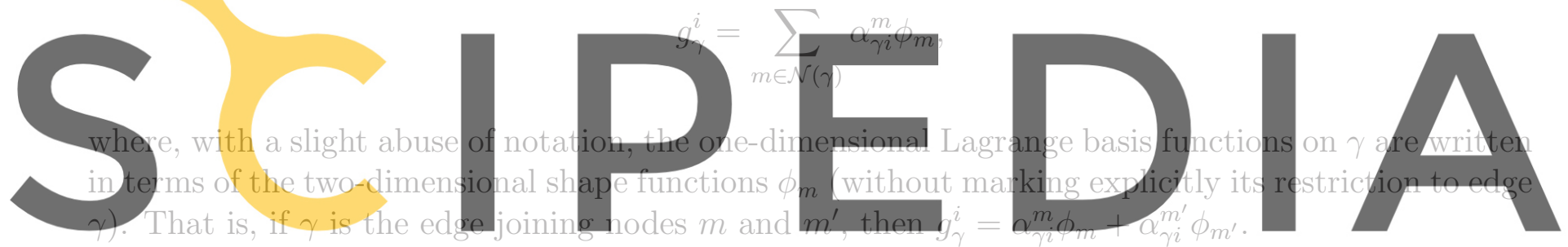

In this case, Appendix C shows that, if the linear tractions verify the weighted projected equilibration Registerdfonfree at https//www.scipedia.com to download the version without the watermark

$$
\int_{\Omega_{k}}\left[\phi_{i}\left(\Pi_{k}^{1} f-\kappa^{2} u_{h}\right)-\nabla u_{h} \cdot \nabla \phi_{i}\right] d \Omega+\sum_{\gamma \subset \partial \Omega_{k}} \int_{\gamma} \sigma_{k}^{\gamma} g_{\gamma}^{i} d \Gamma=0
$$

and assuming that node $\boldsymbol{x}_{[1]}$ coincides with the central node of the star $\boldsymbol{x}_{i}$ so that $\lambda_{[1]}=\phi_{i}$, then a pair of local dual estimates $\boldsymbol{q}_{k}^{i}$ and $r_{k}^{i}$ verifying (18) are obtained by taking

$$
r_{k}^{i}=0
$$

and decomposing the flux $\boldsymbol{q}_{k}^{i}$ into a linear plus a cubic part

$$
\boldsymbol{q}_{k}^{i}=\boldsymbol{q}_{k}^{i L}+\boldsymbol{q}_{k}^{i C}
$$

where

$$
\mathbf{q}_{k}^{i L}=\frac{1}{2\left|\Omega_{k}\right|}\left(\boldsymbol{\rho}_{[1]}^{k} \lambda_{[1]}+\boldsymbol{\rho}_{[2]}^{k} \lambda_{[2]}+\boldsymbol{\rho}_{[3]}^{k} \lambda_{[3]}\right)
$$

for

$$
\begin{aligned}
& \boldsymbol{\rho}_{[1]}^{k}=\left.l_{[3]}\left(\sigma_{k}^{\gamma} g_{\gamma}^{i}-\phi_{i} \boldsymbol{\nabla} u_{h} \cdot \boldsymbol{n}_{k}\right)\right|_{\gamma_{[3]}}\left(\boldsymbol{x}_{[1]}\right) \boldsymbol{t}_{[2]}-\left.l_{[2]}\left(\sigma_{k}^{\gamma} g_{\gamma}^{i}-\phi_{i} \boldsymbol{\nabla} u_{h} \cdot \boldsymbol{n}_{k}\right)\right|_{\gamma_{[2]}}\left(\boldsymbol{x}_{[1]}\right) \boldsymbol{t}_{[3]}, \\
& \boldsymbol{\rho}_{[2]}^{k}=\left.l_{[1]}\left(\sigma_{k}^{\gamma} g_{\gamma}^{i}-\phi_{i} \boldsymbol{\nabla} u_{h} \cdot \boldsymbol{n}_{k}\right)\right|_{\gamma_{[1]}}\left(\boldsymbol{x}_{[2]}\right) \boldsymbol{t}_{[3]}-\left.l_{[3]}\left(\sigma_{k}^{\gamma} g_{\gamma}^{i}-\phi_{i} \boldsymbol{\nabla} u_{h} \cdot \boldsymbol{n}_{k}\right)\right|_{\gamma_{[3]}}\left(\boldsymbol{x}_{[2]}\right) \boldsymbol{t}_{[1]}, \\
& \boldsymbol{\rho}_{[3]}^{k}=\left.l_{[2]}\left(\sigma_{k}^{\gamma} g_{\gamma}^{i}-\phi_{i} \boldsymbol{\nabla} u_{h} \cdot \boldsymbol{n}_{k}\right)\right|_{\gamma_{[2]}}\left(\boldsymbol{x}_{[3]}\right) \boldsymbol{t}_{[1]}-\left.l_{[1]}\left(\sigma_{k}^{\gamma} g_{\gamma}^{i}-\phi_{i} \boldsymbol{\nabla} u_{h} \cdot \boldsymbol{n}_{k}\right)\right|_{\gamma_{[1]}}\left(\boldsymbol{x}_{[3]}\right) \boldsymbol{t}_{[2]}
\end{aligned}
$$


and

$$
\mathbf{q}_{k}^{i C}=\frac{1}{3}\left(\beta_{[1]} \boldsymbol{t}_{[1]} \boldsymbol{t}_{[1]}^{\top}+\beta_{[2]} \boldsymbol{t}_{[2]} \boldsymbol{t}_{[2]}^{\top}+\beta_{[3]} \boldsymbol{t}_{[3]} \boldsymbol{t}_{[3]}^{\top}\right) \nabla v^{Q},
$$

where $\beta_{[1]}=\lambda_{[2]} \lambda_{[3]}, \beta_{[2]}=\lambda_{[1]} \lambda_{[3]}, \beta_{[3]}=\lambda_{[1]} \lambda_{[2]}$ and

$$
v^{Q}=\frac{3}{8} \phi_{i} F+\frac{1}{8}\left(F_{[1]} F_{[2]} F_{[3]}\right)\left(\begin{array}{ccc}
4 & 0 & 0 \\
0 & 0 & -1 \\
0 & -1 & 0
\end{array}\right)\left(\begin{array}{l}
\lambda_{[1]} \\
\lambda_{[2]} \\
\lambda_{[3]}
\end{array}\right),
$$

for $F=\Pi_{k}^{1} f-\kappa^{2} u_{h}=F_{[1]} \lambda_{[1]}+F_{[2]} \lambda_{[2]}+F_{[3]} \lambda_{[3]}$. Note that the notation $F_{[j]}=F\left(\boldsymbol{x}_{[j]}\right)=\Pi_{k}^{1} f\left(\boldsymbol{x}_{[j]}\right)-$ $\kappa^{2} u_{h}\left(\boldsymbol{x}_{[j]}\right), j=1,2,3$ has been used to simplify the expression for the cubic part of the flux $\mathbf{q}_{k}^{i C}$.

Therefore, for given a set of local weighted tractions $\left\{g_{\gamma}^{i}\right\}_{\gamma \subset \omega_{i}}$ verifying both (17) and (20), equations (21) and (22) provide a pair of dual estimates $\boldsymbol{q}_{k}^{i}$ and $r_{k}^{i}$ verifying (16). Note however that conditions (17) and (20) do not determine a unique set of weighted tractions $g_{\gamma}^{i}$. This is because (20) states a condition on each element of the star while the weighted tractions are defined by two degrees of freedom on each edge of the star where neither $\phi_{i}$ vanish nor $\gamma$ belongs to the Neumann boundary. Thus, in order to determine all the unknowns $\left\{g_{\gamma}^{i}\right\}_{\gamma \subset \omega_{i}}$ the set of equations has to be complemented.

Remark 5. It is worth noting that the existence of a set of local weighted tractions $\left\{g_{\gamma}^{i}\right\}_{\gamma \subset \omega_{i}}$ verifying both (17) and (20) is guaranteed by the global compatibility condition (19) which ensures the solvability of both (16) and (18), see Remark 4.

5.4. Objective function of the local optimization problems: minimization of the energy norm
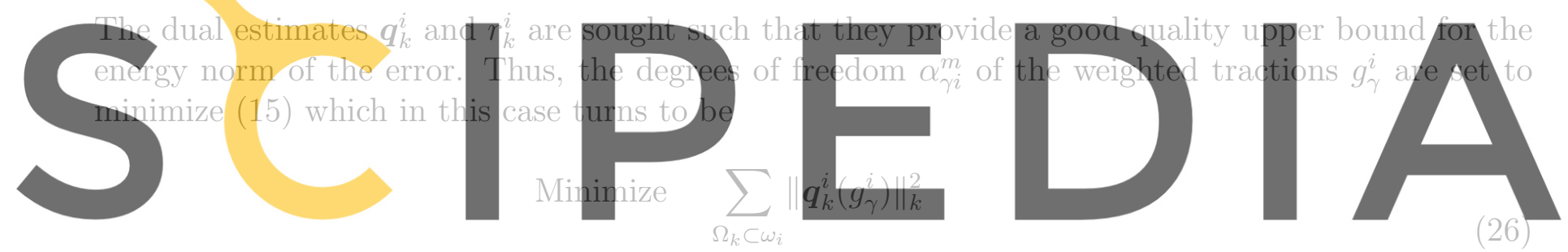

Register for free at https//www.scipedia.com to download the version without the watermark

The local equilibrated fluxes $q_{k}^{i}$ dependency with respect to the tractions $g_{\gamma}^{i}$ is only due to the definition of the linear contribution to the flux, $\boldsymbol{q}_{k}^{i L}$. A positive consequence of this fact is that, the optimization problem (26) turns to be a small constrained quadratic optimization problem. The remainder of the section is devoted to detail the computation of the local weighted tractions by exhaustively describing the constrained quadratic optimization problem to be solved.

It is assumed that $\boldsymbol{x}_{[1]}$ coincides with the central node of the star $\boldsymbol{x}_{i}$. Thus, the tractions in the triangle are expressed as

$$
\begin{aligned}
& g_{\gamma_{[!]}}^{i}=0, \\
& g_{\gamma_{[2]}}^{i}=\alpha_{\gamma_{[2]} i}^{[1]} \lambda_{[1]}+\alpha_{\gamma_{[2]} i}^{[3]} \lambda_{[3]} \text {, } \\
& g_{\gamma_{[3]}}^{i}=\alpha_{\gamma_{3 ; i} i}^{[1]} \lambda_{[1]}+\alpha_{\gamma_{3 \mid i} i}^{[2]} \lambda_{[2]} \text {. }
\end{aligned}
$$

To ease the notation, hereafter the notation for the four unknown tractions coefficients is simplified replacing $\alpha_{\gamma_{j i} i}^{[l]}$ by $\alpha_{j l}$, where the first subindex denotes the local numbering of the edge $\gamma_{[j]}$, the second subscript denotes the local numbering for the node $\boldsymbol{x}_{[l]}$ and the subscript referring to the star $i$ is

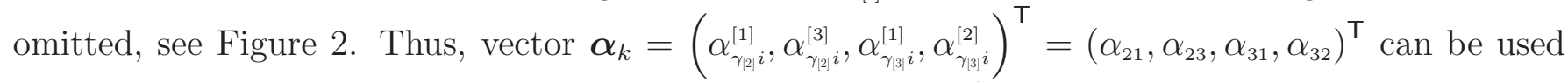
to store the unknown coefficients which define the tractions $g_{\gamma}^{i}$ in element $\Omega_{k}$.

With these notations, the expression for the linear part of the flux $\boldsymbol{q}_{k}^{i L}$ can be simplified noting that 


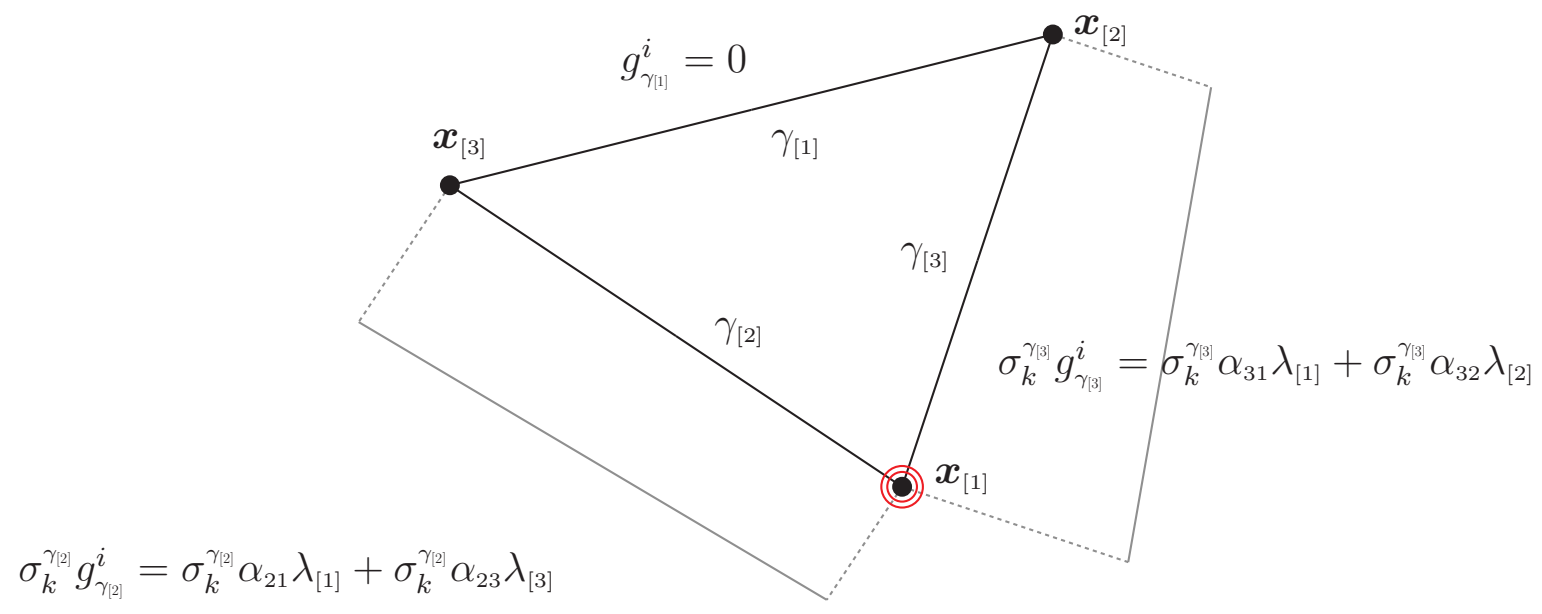

Figure 2: Notation for the weighted tractions $g_{\gamma}^{i}$ in element $\Omega_{k}$ from star $\omega_{i}$.

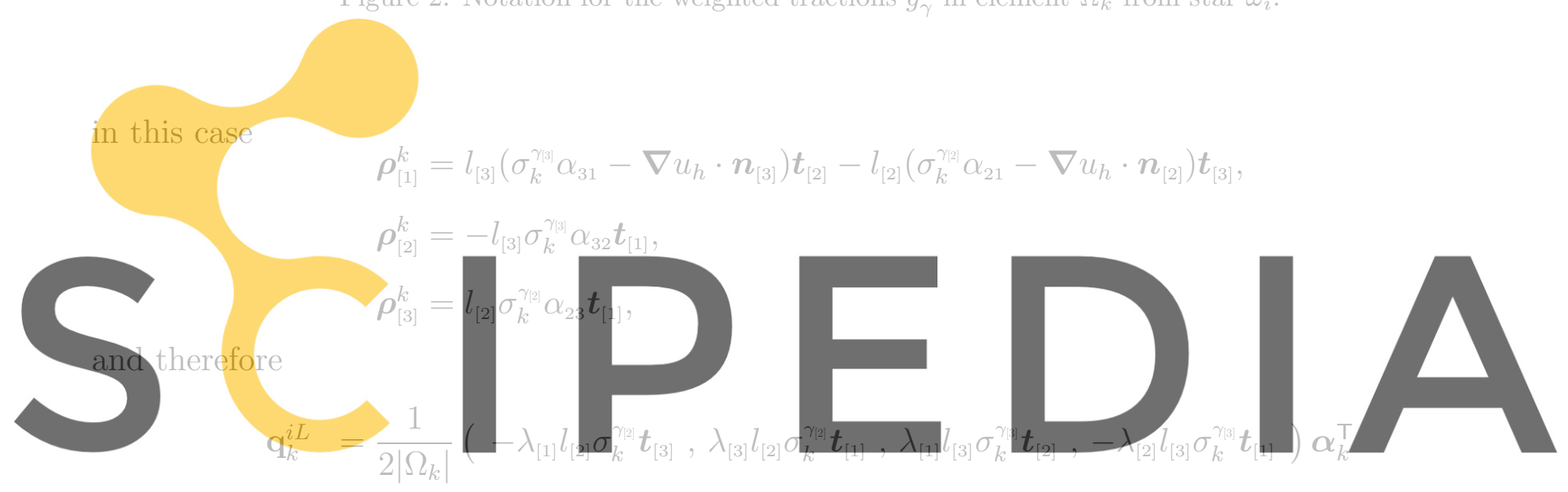

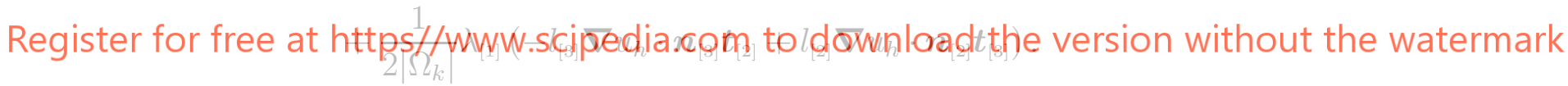

Also, computing the integrals appearing in (20) and multiplying the final result by two, the weighted projected equilibrated condition can be expressed as

$$
\begin{aligned}
& \left(\begin{array}{llll}
l_{[2]} \sigma_{k}^{\gamma_{[2]}} & l_{[2]} \sigma_{k}^{\gamma_{[2]}} & l_{[3]} \sigma_{k}^{\gamma_{[3]}} & l_{[3]} \sigma_{k}^{\gamma_{[3]}}
\end{array}\right) \boldsymbol{\alpha}_{k} \\
& =-\frac{\left|\Omega_{k}\right|}{6}\left(2 F_{[1]}+F_{[2]}+F_{[3]}-12 \nabla u_{h} \cdot \nabla \phi_{i}\right) \text {. }
\end{aligned}
$$

Having a closed expression for the fluxes $\boldsymbol{q}_{k}^{i}$, a closed quadratic formula for its norm depending on the four unknowns given in $\boldsymbol{\alpha}_{k}$ can also be derived. Indeed, the squared norm of the local weighted fluxes is given by

$$
\left\|\boldsymbol{q}_{k}^{i}\right\|_{k}^{2}=\boldsymbol{\alpha}_{k}^{\top} \mathbf{M}_{k}^{L} \boldsymbol{\alpha}_{k}+\boldsymbol{\alpha}_{k}^{\top}\left(\mathbf{b}_{k}^{L}+2 \mathbf{b}_{k}^{L C}\right)+\mathrm{c}_{k}^{L}+2 \mathrm{c}_{k}^{L C}+\mathrm{c}_{k}^{C},
$$

where the expressions for matrix $\mathbf{M}_{k}^{L}$, vectors $\mathbf{b}_{k}^{L}$ and $\mathbf{b}_{k}^{L C}$ and constants $\mathrm{c}_{k}^{L}, \mathrm{c}_{k}^{L C}$ and $\mathrm{c}_{k}^{C}$ are detailed in Appendix D.

\subsection{Brief review of the algorithm to compute the upper bounds for $\||\|\mid\|$}

The following chart describes the steps to compute upper bounds for $\|e\| \|$ using the new cheaper flux-free approach. The importance of the closed expressions both for the weighted fluxes and their norms is that it reduces the problem of computing upper bounds for $\|e\|$ to solving a small quadratic optimization problem. The procedure is sketched as follows: 
1. For each node of the mesh $\boldsymbol{x}_{i}$, consider its associated star $\omega_{i}$.

(a) Consider the global vector $\boldsymbol{\alpha}^{i}$ containing all the traction unknowns associated to the edges in $\Gamma_{i}$, that is, $\boldsymbol{\alpha}^{i}=\left[\alpha_{\gamma_{j} i}^{m}\right]_{\gamma_{j} \in \Gamma_{i}, m \in \mathcal{N}\left(\gamma_{j}\right)}$ contains $2 \times \operatorname{cardinal}\left(\Gamma_{i}\right)$ unknowns.

(b) For each element of the star $\Omega_{k} \subset \omega_{i}$, compute the matrices and vectors

$$
\mathbf{M}_{k}^{L}, \mathbf{b}_{k}^{L} \text { and } \mathbf{b}_{k}^{L C}
$$

associated to the local unknowns $\boldsymbol{\alpha}_{k}=\left(\alpha_{21}, \alpha_{23}, \alpha_{31}, \alpha_{32}\right)^{\top}$, and assemble these contributions to the global matrices

$$
\mathbf{M}_{\omega_{i}}^{L}, \mathbf{b}_{\omega_{i}}^{L} \text { and } \mathbf{b}_{\omega_{i}}^{L C}
$$

associated to the global unknowns $\boldsymbol{\alpha}^{i}$.

(c) For each element of the star $\Omega_{k} \subset \omega_{i}$, compute the weighted projected equilibrated condition (27). Assemble all the conditions into the linear global system of equations

$$
\mathbf{A}_{\omega_{i}} \boldsymbol{\alpha}^{i}=\mathbf{b}_{\omega_{i}}
$$

(d) For each edge $\gamma$ of element $\Omega_{k} \subset \omega_{i}$ lying on the Neumann boundary, impose the boundary conditions (17b) and add these restrictions to the global system of equations given in (29). Solve the following quadratic optimization problem with only equality linear constraints

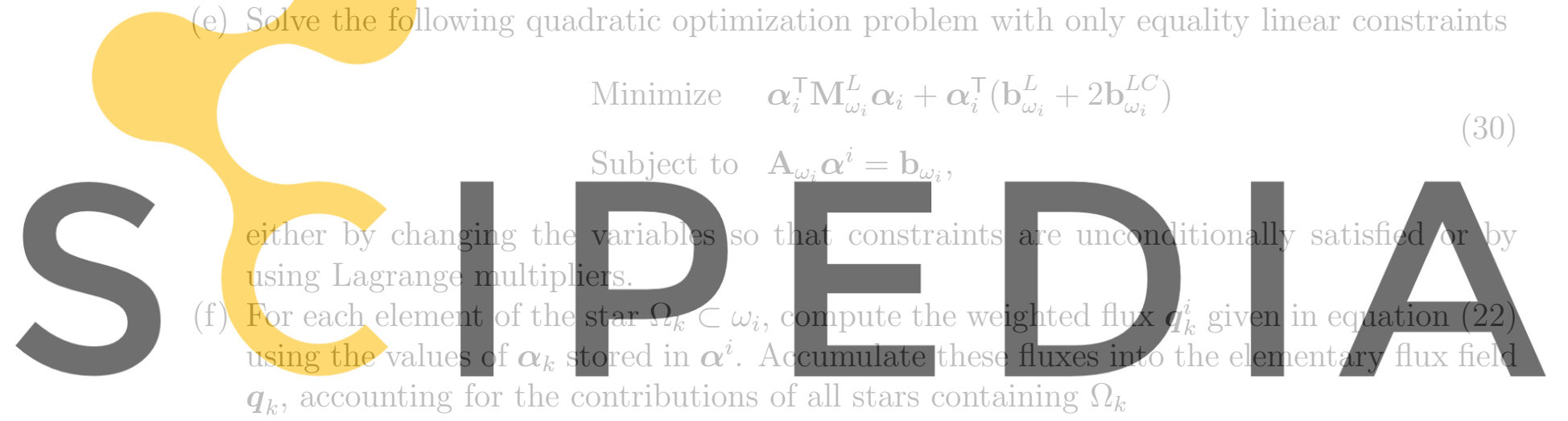

Register for free at https//www.scipedia.com to download the version without the watermark $q_{k}=\sum_{i \in \mathcal{N}\left(\Omega_{k}\right)} q_{k}^{2}$

2. For each element of the mesh $\Omega_{k}$

(a) Compute the data oscillations terms $\operatorname{osc}_{k}(f)$ and $\operatorname{osc}_{\gamma}\left(g_{\mathrm{N}}\right)$ associated to $\hat{q}=1$ and $\bar{q}=0$.

(b) Compute the local norm contribution $\left\|\boldsymbol{q}_{k}\right\|_{k}$ and set $\left\|r_{k}\right\|_{k}=0$.

(c) Compute the local error contribution $\eta_{k}$ defined in equation (9) and add this contribution to the upper bound for the error.

3. Return the upper bound for the norm of the error given by

$$
\eta=\left(\sum_{k=1}^{n_{\mathrm{el}}} \eta_{k}^{2}\right)^{1 / 2}
$$

\subsection{Computational aspects}

The local norm contribution $\left\|\boldsymbol{q}_{k}\right\|_{k}$ can be computed in many different ways. One option is to use standard finite element matrices since $\boldsymbol{q}_{k}^{i}$ and $\boldsymbol{q}_{k}$ are cubic fluxes as shown in [20]. Let $\Omega_{k}$ be the triangle defined by the vertices $\boldsymbol{x}_{[1]}, \boldsymbol{x}_{[2]}$ and $\boldsymbol{x}_{[3]}$, and consider a cubic interpolation for the fluxes $\boldsymbol{q}_{k}$ defined using the standard Lagrange cubic basis functions. Denote by $\boldsymbol{z}_{[j]}$ and $\lambda_{[j]}^{c}$ the points and Lagrange basis functions describing this interpolation, see Figure 3. Then the flux $\boldsymbol{q}_{k}$ can be expressed as $\boldsymbol{q}_{k}=\sum_{j=1}^{10} \boldsymbol{q}_{k}\left(\boldsymbol{z}_{[j]}\right) \lambda_{[j]}^{c}$, and the local norm of the flux $\boldsymbol{q}_{k}$ can be computed as $\left\|\boldsymbol{q}_{k}\right\|_{k}^{2}=\left|\Omega_{k}\right| \mathbf{q}_{k}^{\top} \mathbf{M}_{\mathrm{el}}^{c} \mathbf{q}_{k}$ 

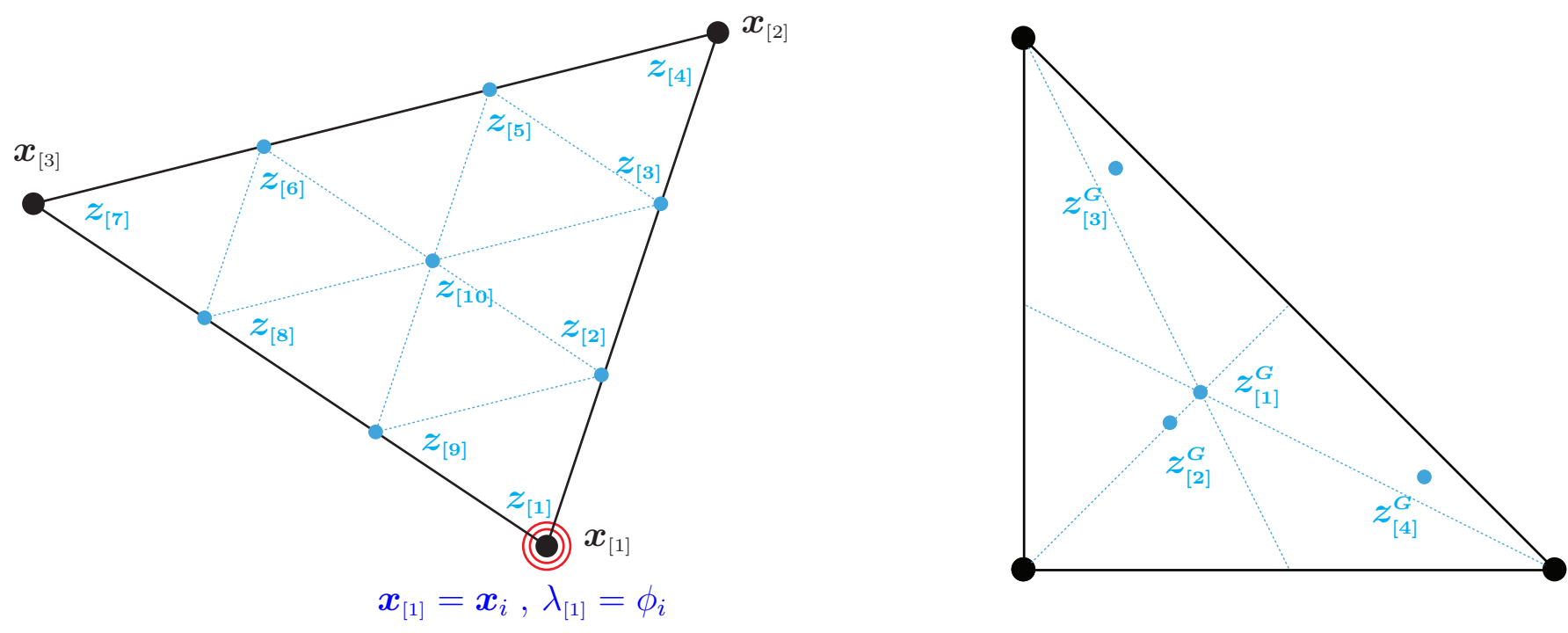

Figure 3: Notation for the nodes describing of a cubic flux in an element (left) and Gauss points exactly integrating the square of a cubic polynomial (right).

where the local vector describing $\boldsymbol{q}_{k}$ is given by $\left.\mathbf{q}_{k}^{\top}=\left(\begin{array}{lll}\boldsymbol{q}_{k}\left(\boldsymbol{z}_{[1]}\right)^{\top} & \ldots & \boldsymbol{q}_{k}\left(\boldsymbol{z}_{[10]}\right.\end{array}\right)^{\top}\right)$ and $\mathbf{M}_{\mathrm{el}}^{c}$ is the symmetric block mass matrix given by

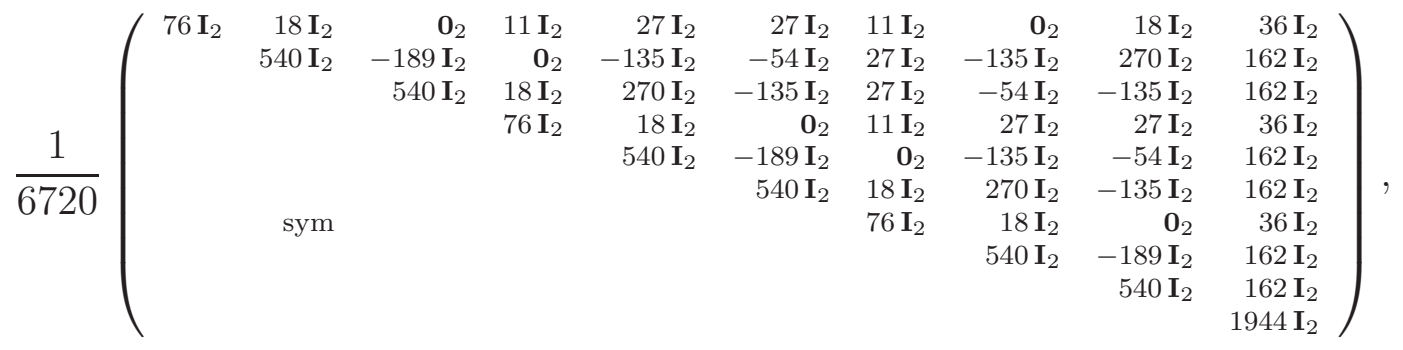

where $\mathbf{I}_{2}$ and $\mathbf{0}_{2}$ are the two-by-two identity and zero matrices respectively.

However, in this case, since the flux $\boldsymbol{q}_{k}$ (and also its star contribution $\boldsymbol{q}_{k}^{i}$ ) is only needed in the computation of the local norm $\left\|\boldsymbol{q}_{k}\right\|_{k}$ - composed of squares of cubic polynomials - a more efficient strategy only storing the values of the fluxes at four appropriate Gauss points (like the ones given in Table 1 for the reference unitary triangle) instead of the ten finite element nodes is proposed. Specifically, in step 1(f), for each element of the star $\Omega_{k} \subset \omega_{i}$, the value of the weighted flux $\boldsymbol{q}_{k}^{i}$ at the Gauss points $\boldsymbol{z}_{[i]}^{G}, i=1, \ldots, 4$ given in Figure 3 and Table 1 are computed using equation (22) and these fluxes are accumulated using equation (31) into the elementary flux field $\boldsymbol{q}_{k}$. Finally, the norm is exactly computed as

$$
\left\|\boldsymbol{q}_{k}\right\|_{k}^{2}=\frac{\left|\Omega_{k}\right|}{2} \sum_{i=1}^{4} w_{[i]}^{G} \boldsymbol{q}_{k}\left(\boldsymbol{z}_{[i]}^{G}\right)^{\top} \boldsymbol{q}_{k}\left(\boldsymbol{z}_{[i]}^{G}\right) .
$$

\begin{tabular}{c|c} 
Gauss point $\boldsymbol{z}_{[i]}^{G}$ & weight $w_{[i]}^{G}$ \\
\hline$(0.33333333333333333333,0.33333333333333333333)$ & 0.14438502673796791444 \\
$(0.27602622373694168712,0.27602622373694168712)$ & 0.47388781431334622824 \\
$(0.17390604578891849847,0.75482228851436454027)$ & 0.19086357947434292866 \\
$(0.75482228851436454027,0.17390604578891849847)$ & 0.19086357947434292866
\end{tabular}

Table 1: Gaussian quadrature exact for squares of cubic polynomials in two dimensions. 


\section{Alternative guaranteed upper bound computation: a new more efficient equilibrated residual method}

The equilibrated residual method is based on solving a local boundary value problem in each element of the mesh where the source term is given by the strong residual in the element interior and suitable Neumann boundary conditions are imposed on the element boundary. These Neumann boundary conditions correspond to the equilibrated tractions and, for element $\Omega_{k}$ of the mesh, are denoted by $g_{k}$.

Once the equilibrated tractions are computed, the error $e=u-u_{h}$ in element $\Omega_{k}$ is approximated by: $\hat{e}_{k} \in \mathcal{V}\left(\Omega_{k}\right)$ such that

$$
a_{k}\left(\hat{e}_{k}, v\right)=\ell_{k}^{\Pi}(v)-a_{k}\left(u_{h}, v\right)+\int_{\partial \Omega_{k} \backslash \Gamma_{\mathrm{N}}} g_{k} v d \Gamma \quad \text { for all } v \in \mathcal{V}\left(\Omega_{k}\right),
$$

where $a_{k}(\cdot, \cdot)$ and $\ell_{k}^{\Pi}(\cdot)$ are the restrictions of $a(\cdot, \cdot)$ and $\ell^{\Pi}(\cdot)$ to $\Omega_{k}$.

If the tractions $g_{k}$ are chosen such that: 1) they coincide with the projected Neumann data on $\Gamma_{\mathrm{N}}$, $\left.g_{k}\right|_{\gamma}=\Pi_{\gamma}^{\bar{q}} g_{\mathrm{N}}$ on $\left.\gamma \subset \Gamma_{\mathrm{N}}, 2\right)$ they verify the consistency conditions

$$
g_{k}+g_{k^{\prime}}=0 \text { on } \Omega_{k} \cap \Omega_{k^{\prime}}
$$

and 3) they verify the projected equilibration conditions (zeroth-order equilibration conditions [1])

$$
\int_{\Omega_{k}} \Pi_{k}^{\hat{q}} f d \Omega-a_{k}\left(u_{h}, 1\right)+\int_{\partial \Omega_{k}} g_{k} d \Gamma=0
$$

then, the solution of the local problem (32) exists for all $\kappa \geq 0$, and the following upper bound follows

$$
\|e\|^{2} \leq \sum_{k=1}^{n_{\mathrm{el}}}\left[\left\|\hat{e}_{k}\right\|_{k}+\operatorname{osc}_{k}(f)+\sum_{\gamma \subset \Gamma_{\mathrm{N}} \cap \partial \Omega_{k}} \operatorname{osc}_{\gamma}\left(g_{\mathrm{N}}\right)\right]^{2} .
$$

A vast literature exists providing different approaches to compute the equilibrated tractions, see for instance $[1,11,10,21,23,12]$. The standard computation of the equilibrated tractions involves solving a local problem for each node $\boldsymbol{x}_{i}$ of the mesh where the zeroth-order equilibration conditions (34) are imposed via the more restrictive first-order equilibration conditions

$$
\int_{\Omega_{k}} \Pi_{k}^{\hat{q}} f \phi_{i} d \Omega-a_{k}\left(u_{h}, \phi_{i}\right)+\int_{\partial \Omega_{k}} g_{k} \phi_{i} d \Gamma=0
$$

which ensures (34) since the sum of the first-order basis functions is the constant unit function. Additionally, since conditions (35) do not uniquely determine a linear set of equilibrated tractions, $g_{k}$ are set to be as close as possible to the tractions provided by the finite element method (averaged over the edges of the mesh), namely $\left.g_{k}\right|_{\gamma} \approx 1 / 2\left(\left.\nabla u_{h}\right|_{\Omega_{k}}+\left.\nabla u_{h}\right|_{\Omega_{k^{\prime}}}\right) \cdot \boldsymbol{n}_{k}$ for $\gamma=\Omega_{k} \cap \Omega_{k^{\prime}}$, see [1] for a detailed explanation.

This section shows that the new technique presented in the previous sections, provides a new methodology to obtain cheap and efficient equilibrated tractions. Following the notations introduced in [10] this new equilibrated technique would be classified as a new element equilibrated + star patch technique (EESPT).

Indeed, let $\left\{g_{\gamma}^{i}\right\}_{i \in \mathcal{N}}$ be a set of local weighted tractions verifying equations (17) and (20) and consider the global tractions obtained by adding all the weighted contributions, namely

$$
g_{\gamma}=\sum_{i=1}^{n_{\mathrm{np}}} g_{\gamma}^{i}
$$


Note that, as is the case of the star traction $g_{\gamma}^{i}$, the traction $g_{\gamma}$ is associated to the arbitrary but fixed unit normal direction $\boldsymbol{n}^{\gamma}$. Then, for some element $\Omega_{k}$ and one of its edges $\gamma \subset \partial \Omega_{k}$, one can define the approximation of the exact tractions on the interface $\gamma$ of element $\Omega_{k}$ as

$$
\left.g_{k}\right|_{\gamma}=\sigma_{k}^{\gamma} g_{\gamma}
$$

Then, as the following Theorem states, the set of tractions $\left\{g_{k}\right\}_{k=1, \ldots, n_{\mathrm{el}}}$ defined in (37) are equilibrated.

Theorem 2. The set of tractions $\left\{g_{k}\right\}_{k=1, \ldots, n_{\mathrm{el}}}$ defined using equations (37), (36), (17) and (20) are equilibrated, that is, they coincide with the projected Neumann data in $\Gamma_{\mathrm{N}}$ for $\bar{q}=0$, they verify the consistency conditions (33) and they also verify the projected equilibration conditions (34) for $\hat{q}=1$.

Proof Let $\gamma \in \Gamma_{\mathrm{N}}$ be an edge in the Neumann boundary, then from (17b) and using the partition of unity of the linear shape functions

$$
g_{\gamma}=\sum_{i=1}^{n_{\mathrm{np}}} g_{\gamma}^{i}=\sum_{i=1}^{n_{\mathrm{np}}} \phi_{i} \Pi_{\gamma}^{0} g_{\mathrm{N}}=\Pi_{\gamma}^{0} g_{\mathrm{N}}
$$

which ensures that $\left.g_{k}\right|_{\gamma}=\Pi_{\gamma}^{0} g_{\mathrm{N}}$ since in the boundary of the domain $\sigma_{k}^{\gamma}=1$.

Let $\gamma=\partial \Omega_{k} \cap \partial \Omega_{k^{\prime}}$. Then, $\left.g_{k}\right|_{\gamma}+\left.g_{k^{\prime}}\right|_{\gamma}=\left(\sigma_{k}^{\gamma}+\sigma_{k^{\prime}}^{\gamma}\right) g_{\gamma}=\left(\boldsymbol{n}_{k}^{\gamma}+\boldsymbol{n}_{k^{\prime}}^{\gamma}\right) \cdot \boldsymbol{n}^{\gamma} g_{\gamma}=0$ and the consistency condition (33) is verifyied.

In order to see if the tractions are equilibrated, note that the projected equilibration condition (34) for $\hat{q}=1$ and $g_{k}$ defined in (37) can be rewritten as

$$
\int_{\Omega_{k}}\left(\Pi_{k}^{1} f-\kappa^{2} u_{h}\right) d \Omega+\sum_{\gamma \subset \partial \Omega_{k}} \int_{\gamma} \sigma_{k}^{\gamma} g_{\gamma} d \Gamma=0 .
$$

Then, the projected weighted equilibration conditions (20) immediately imply that the tractions are equilibrated, since for each element, equation (38) is recovered by adding all the contributions from the stars to this element and using the partition of unity of the shape functions.

Remark 6. There are two main differences between the standard equilibration procedure and the novel one. First, note that the first-order equilibration conditions given in (35) and (20) differ only in the boundary term, where in the standard technique the tractions in element $\Omega_{k}$ and edge $\gamma$ are given by $\left.g_{k} \phi_{i}\right|_{\gamma}$ while in the novel approach they are given by $\sigma_{k}^{\gamma} g_{\gamma}^{i}$. That is, for an edge $\gamma$ joining nodes $\boldsymbol{x}_{i}$ and $\boldsymbol{x}_{i^{\prime}}$, the standard technique uses only $\left.g_{k}\right|_{\gamma}$ in (35) weighted by $\phi_{i}$ and $\phi_{i^{\prime}}$ when associated with nodes $\boldsymbol{x}_{i}$ and $\boldsymbol{x}_{i^{\prime}}$, respectively. On the other hand, the novel approach decomposes the tractions on an edge into two independent linear contributions $g_{\gamma}^{i}$ and $g_{\gamma}^{i^{\prime}}$, each one used separately in the corresponding condition (20). The second difference is the optimization procedure enforced to obtain a unique set of linear tractions. While the standard approach enforces the tractions to be similar to the finite element method averaged tractions, the novel approach computes the tractions solving the optimization problem (26). Note that the essential feature of the new method is that it doubles the degrees of freedom per edge of the mesh so that an improved local optimization procedure is used to obtain very accurate equilibrated tractions.

Given the equilibrated tractions $g_{\gamma}$, there are many different a posteriori error estimation strategies that can be used to compute guaranteed upper bounds for the energy norm of the error $\||\|\mid\|$, see for instance $[24,25,26]$.

For instance, one of the most used strategies is to approximate (32) introducing a finite element submesh $\mathcal{V}^{h} \subset \mathcal{V}^{h^{\prime}}$ to obtain an estimate $\hat{e}_{k}^{h^{\prime}} \in \mathcal{V}^{h^{\prime}}\left(\Omega_{k}\right)$ verifying

$$
\left\|e_{h^{\prime}}\right\|^{2} \leq \sum_{k=1}^{n_{\mathrm{el}}}\left[\left\|\hat{e}_{k}^{h^{\prime}}\right\| \|_{k}+\operatorname{osc}_{k}(f)+\sum_{\gamma \subset \Gamma_{\mathrm{N}} \cap \partial \Omega_{k}} \operatorname{osc}_{\gamma}\left(g_{\mathrm{N}}\right)\right]^{2},
$$


where $e_{h^{\prime}}=u_{h^{\prime}}-u_{h}$ is the reference error. Note that since $\left\|e_{h^{\prime}}\right\| \leq\|\| e \|$, the previous upper bound is not guaranteed for the exact error but just for the reference, and therefore, only an asymptotic upper bound for the exact error is obtained.

In order to obtain guaranteed upper bounds for the exact error, a dual approach is used [24, 25]. In this case, instead of aiming to compute $\hat{e}_{k} \in \mathcal{V}\left(\Omega_{k}\right)$ verifying equation (32) the problem is reduced to finding $\boldsymbol{q}_{k} \in\left[\mathbb{P}^{q}\left(\Omega_{k}\right)\right]^{2}$ and $r_{k} \in \mathbb{P}^{q}\left(\Omega_{k}\right)$ such that for all $v \in \mathcal{V}\left(\Omega_{k}\right)$ it holds that

$$
\int_{\Omega_{k}}\left[\boldsymbol{q}_{k} \cdot \nabla v+\kappa^{2} r_{k} v\right] d \Omega=\ell_{k}^{\Pi}(v)-a_{k}\left(u_{h}, v\right)+\int_{\partial \Omega_{k} \backslash \Gamma_{\mathrm{N}}} g_{k} v d \Gamma
$$

and such that the resulting fields $\boldsymbol{q}_{k}$ and $r_{k}$ have minimum $\mathcal{L}^{2}$-norm. It is easy to see that

$$
\sum_{k=1}^{n_{\mathrm{el}}} \int_{\Omega_{k}}\left[\boldsymbol{q}_{k} \cdot \nabla v+\kappa^{2} r_{k} v\right] d \Omega=\ell^{\Pi}(v)-a\left(u_{h}, v\right) \quad \text { for all } v \in \mathcal{V},
$$

and therefore, the global dual estimates $\boldsymbol{q} \in\left[\mathcal{L}^{2}(\Omega)\right]^{2}$ and $r \in \mathcal{L}^{2}(\Omega)$ such that $\left.\boldsymbol{q}\right|_{\Omega_{k}}=\boldsymbol{q}_{k}$ and $\left.r\right|_{\Omega_{k}}=r_{k}$ verify equation (8) and provide a guaranteed upper bound for the energy norm of the error.

Alternatively, in the case of the tractions $g_{k}$ being linear, and if one considers either piecewise constant or piecewise linear projections for the data, that is, $\hat{q}$ and $\bar{q}$ are at most one, one could use the approach presented in [2]. It proposes to compute $\boldsymbol{q}_{k}$ and $r_{k}$ verifying (39) but not minimizing $\left\|\boldsymbol{q}_{k}\right\|_{k}^{2}+\kappa^{2}\left\|r_{k}\right\|_{k}^{2}$. The resulting bounds are worst that the ones proposed in [25], but this alternative does not require solving any local residual problem. Indeed, under these assumptions one can take $r_{k}$ to be zero in all the elements of the mesh and consider the following closed expression for $\boldsymbol{q}_{k}$ :

$$
\boldsymbol{q}_{k}=\boldsymbol{q}_{k}^{L}+\boldsymbol{q}_{k}^{Q},
$$

where

$$
\mathbf{q}_{k}^{L}=\frac{1}{2\left|\Omega_{k}\right|}\left(\boldsymbol{\rho}_{[1]}^{k} \lambda_{[1]}+\boldsymbol{\rho}_{[2]}^{k} \lambda_{[2]}+\boldsymbol{\rho}_{[3]}^{k} \lambda_{[3]}\right)
$$

for

$$
\begin{aligned}
& \boldsymbol{\rho}_{[1]}^{k}=\left.l_{[3]}\left(g_{k}-\nabla u_{h} \cdot \boldsymbol{n}_{k}\right)\right|_{\gamma_{[3]}}\left(\boldsymbol{x}_{[1]}\right) \boldsymbol{t}_{[2]}-\left.l_{[2]}\left(g_{k}-\nabla u_{h} \cdot \boldsymbol{n}_{k}\right)\right|_{\gamma_{[2]}}\left(\boldsymbol{x}_{[1]}\right) \boldsymbol{t}_{[3]}, \\
& \boldsymbol{\rho}_{[2]}^{k}=\left.l_{[1]}\left(g_{k}-\nabla u_{h} \cdot \boldsymbol{n}_{k}\right)\right|_{\gamma_{[1]}}\left(\boldsymbol{x}_{[2]}\right) \boldsymbol{t}_{[3]}-\left.l_{[3]}\left(g_{k}-\nabla u_{h} \cdot \boldsymbol{n}_{k}\right)\right|_{\gamma_{[3]}}\left(\boldsymbol{x}_{[2]}\right) \boldsymbol{t}_{[1]}, \\
& \boldsymbol{\rho}_{[3]}^{k}=\left.l_{[2]}\left(g_{k}-\boldsymbol{\nabla} u_{h} \cdot \boldsymbol{n}_{k}\right)\right|_{\gamma_{[2]}}\left(\boldsymbol{x}_{[3]}\right) \boldsymbol{t}_{[1]}-\left.l_{[1]}\left(g_{k}-\boldsymbol{\nabla} u_{h} \cdot \boldsymbol{n}_{k}\right)\right|_{\gamma_{[1]}}\left(\boldsymbol{x}_{[3]}\right) \boldsymbol{t}_{[2]}
\end{aligned}
$$

and

$$
\mathbf{q}_{k}^{Q}=\frac{1}{3}\left(\beta_{[1]} \boldsymbol{t}_{[1]} \boldsymbol{t}_{[1]}^{\top}+\beta_{[2]} \boldsymbol{t}_{[2]} \boldsymbol{t}_{[2]}^{\top}+\beta_{[3]} \boldsymbol{t}_{[3]} \boldsymbol{t}_{[3]}^{\top}\right) \nabla\left(\Pi_{k}^{1} f-\kappa^{2} u_{h}\right) .
$$

\section{Computational cost overview}

This section briefly compares the computational effort required to solve the local problems of the presented approach with respect to: M1) guaranteed upper bound based on [20, 18], where the local optimization problems given by equations (15) and (16) are solved directly minimizing the norm with respect to the unknown piecewise-cubic polynomial field $\boldsymbol{q}^{i}$, and M2) guaranteed upper bound based on [2], where first the standard equilibrated tractions are computed and then the upper bound is computed adding the local norm contributions $\left\|\boldsymbol{q}_{k}\right\|_{k}$, where $\boldsymbol{q}_{k}$ is computed as shown in equation (40). In all the cases, the cost of computing a strict upper bound for the energy norm of the error is governed by the cost of the local systems of equations that have to be solved, and therefore, the cost of explicitly evaluating the dual fluxes $\boldsymbol{q}_{k}$ and computing its norm is not incorporated in the computational cost. Additionally, when the solution of a constrained optimization problem is needed the computational cost is evaluated, assuming that the Lagrange multiplier method is used to enforce 
the constraints. Of course, this is not an efficient implementation of the local problems and the cost of the algorithms involving these optimization strategies could be reduced selecting a more efficient strategy to enforce the constraints.

Let $\omega_{i}$ be an interior star (not intersecting the boundary of the domain), and denote by $m$ the number of elements in this star $\omega_{i}$. Reference [18] specifies the computational cost of the local problems associated to error estimate M1 in terms of the interpolation degree $q$. This cost is displayed in Table 2. Regarding the cost of the error estimate M2, it is well known that the computation of the standard equilibrated tractions involves solving a local optimization problem for each star of the mesh, with one degree of freedom per interior edge of the star ( $m$ edges) and $m$ constraints (one of which is linearly dependent). Finally, the error estimate described in Sections 5 and 6 requires solving a local optimization problem for each star of the mesh, see equation (30), with two degrees of freedom per interior edge of the star and one constraint per element of the star.

\begin{tabular}{c|c|ccc|c} 
method & $q$ & d.o.f. & constraints & d.o.f. lagr. mult. & linear solver \\
\hline M1 & 2 & $12 m$ & $9 m-1$ & $21 m-1$ & $9261 m^{3}$ \\
& 3 & $20 m$ & $14 m-1$ & $34 m-1$ & $39304 m^{3}$ \\
\hline M2 & 2 & $m$ & $m$ & $2 m$ & $8 m^{3}$ \\
\hline new & 3 & $2 m$ & $m$ & $3 m$ & $27 m^{3}$ \\
\hline
\end{tabular}

Table 2: Number of degrees of freedom of the local problems for an interior star with $m$ elements an approximation of the computational cost of the local optimization problems using a standard linear solver.

\section{Numerical examples}

The behavior of the new flux-free equilibrated strategy presented above is analyzed in three numerical examples. Some of the selected examples have been used by other authors to assess the performance of different error estimation techniques both for the Poisson problem and for the reaction-diffusion equation $[20,24,13,22,4,2]$.

When reporting the results, let $\eta$ be the upper bound of the energy norm of the error defined in Section 5 and let $\hat{\eta}$ be the upper bound obtained from the new equilibrated tractions by using $r_{k}=0$ and $\boldsymbol{q}_{k}$ defined in (40) in the expression for the local error contribution (9). Also let $\eta^{q}$ be the upper bound of the energy norm of the error computed using new quadratic equilibrated tractions. These new quadratic tractions are computed using a parallel approach to the procedure to compute the new linear tractions, and its computation is briefly described in Appendix E. These new guaranteed upper bounds are compared with the following ones:

$\eta_{1}^{\text {eq }}$ : guaranteed upper bound based on [25], where first the standard equilibrated tractions are computed and then the local elementary problems posed in (39) are solved using cubic polynomial functions for $\boldsymbol{q}_{k}$ and $r_{k}$. Here, the upper bound is computed including the data oscillation term, equation (9), even though this was not considered in the original work

$\eta_{2}^{\text {eq }}$ : guaranteed upper bound based on [2], where first the standard equilibrated tractions are computed and then the upper bound is computed adding the local norm contributions $\left\|\boldsymbol{q}_{k}\right\|_{k}$, where $\boldsymbol{q}_{k}$ is computed as shown in equation (40)

$\eta^{\text {st }}$ guaranteed upper bound based on [18], where the local optimization problems (18) are not solved using the explicit formula for $\boldsymbol{q}^{i}$ and then minimizing with respect to the local tractions unknowns, but directly minimizing the norm with respect to the unknown piecewise-cubic polynomial field $\boldsymbol{q}^{i}$. 
It is worth noting that by construction the following relation holds

$$
\|\mid\| \leq \eta_{1}^{\mathrm{eq}} \leq \eta_{2}^{\mathrm{eq}}
$$

Also, it is expected that in most cases

$$
\|\mid\| \leq \eta^{\text {st }} \leq \eta^{q} \leq \eta
$$

but these last inequalities may not be fulfilled since the estimates are constructed minimizing the squared norm of the fluxes $\boldsymbol{q}_{k}^{i}$ in each star, whereas the global upper bound is computed by first adding the estimates $\boldsymbol{q}_{k}^{i}$ and then computing its squared norm.

These relations express that, as the computational cost is reduced, the bounds may loose efficiency. However, as shown in the numerical examples, this reduction is not very significant and therefore, the cheapest strategies are recommended.

In the examples where the analytical solution is known, the quality of the error estimates is measured using the standard effectivity index

$$
\rho^{*}=\eta^{*} /\|e\| \| \text {. }
$$

All the previous estimates provide local error information which can be used as an indicator for mesh adaptivity. The square of the estimate $\eta$ (an analogously all the other estimates) can be split into the local elemental contributions $\eta_{k}$ given in (9)

$$
\eta^{2}=\sum_{k=1}^{n_{\mathrm{el}}} \eta_{k}^{2}
$$

Then, the elemental contributions $\eta_{k}^{2}$ can serve as informative mesh adaptivity indicators for controlling the error in the energy norm. Note that these indicators also take into account the data oscillation errors, and therefore, the mesh is refined both in the areas most contributing to the error and in the areas where the data cannot be properly represented using its linear/constant projection.

\subsection{Uniformly Forced Square Domain}

A simple diffusion model for the temperature distribution $u(x, y)$ in a square plate $\Omega=(-1,1) \times$ $(-1,1)$ is considered. The specific source term in this example models uniform heating of the plate $f=1$, and the boundary condition models the edge of the plate being kept at an ice-cold temperature, that is, homogeneous Dirichlet boundary conditions are considered all along the boundary.

The simple shape of the domain enables the solution to be explicitly represented as

$$
u(x, y)=\frac{1-x^{2}}{2}-\frac{16}{\pi^{3}} \sum_{\text {odd } k=1}^{\infty} \frac{\sin (k \pi(1+x) / 2)(\sinh (k \pi(1+y) / 2)+\sinh (k \pi(1-y) / 2))}{k^{3} \sinh (k \pi)} .
$$

The convergence of the bounds is analyzed for a uniform mesh refinement in a series of structured meshes. The initial mesh is composed of 8 triangular elements (half squares) and in each refinement step every triangle is divided into four similar triangles. The results can be found in Table 3 . As it has been shown for instance in the series $[15,20,18,19,7,6]$ the flux-free strategies are really competitive with respect to the equilibrated strategies, which can be again confirmed in Table 3 comparing the effectivities $\rho^{\text {st }}$ versus $\rho_{1}^{\text {eq }}$. The new information that is presented in this table is that, when no reaction term is present in the problem, substantially alleviating the cost of the flux-free strategy by either using $\eta$ or $\hat{\eta}$ instead of $\eta^{\text {st }}$ does not significantly modify the value of the effectivites. Of course, introducing quadratic tractions in $\rho^{q}$ provides really close results to the third order tractions implicitly computed in $\rho^{\text {st }}$. However, since the difference between the results obtained using linear or quadratic tractions is really small, there is no need to increase the computational cost. 


\begin{tabular}{c||c||ccc|c||cc}
\hline \multicolumn{1}{l||}{} & \multicolumn{1}{c||}{} & \multicolumn{4}{c||}{ flux-free } & \multicolumn{2}{c}{ equilibrated } \\
\hline$n_{\mathrm{el}}$ & \|\|$e \|$ & $\rho$ & $\hat{\rho}$ & $\rho^{q}$ & $\rho^{\text {st }}$ & $\rho_{1}^{\text {eq }}$ & $\rho_{2}^{\text {eq }}$ \\
\hline 8 & 0.34331271 & 1.09131 & 1.05418 & 1.01545 & 1.00036 & 1.20880 & 1.37311 \\
32 & 0.27603795 & 1.05288 & 1.04760 & 1.03831 & 1.04611 & 1.48894 & 1.50945 \\
128 & 0.15288301 & 1.04621 & 1.04472 & 1.03889 & 1.04314 & 1.51749 & 1.52837 \\
512 & 0.07856757 & 1.04470 & 1.04422 & 1.03938 & 1.04088 & 1.52104 & 1.52704 \\
2048 & 0.03955958 & 1.04429 & 1.04413 & 1.03962 & 1.03948 & 1.51898 & 1.52223 \\
8192 & 0.01980831 & 1.04420 & 1.04414 & 1.03974 & 1.03862 & 1.51641 & 1.51813 \\
32768 & 0.00990510 & 1.04419 & 1.04417 & 1.03982 & 1.03813 & 1.51453 & 1.51542 \\
\hline
\end{tabular}

Table 3: Example 1: effectivities of the upper bounds for the error in the energy norm in a series of uniformly $h$-refined linear triangular meshes.

The reduction of cost in the standard equilibrated strategies, when comparing $\rho_{1}^{\mathrm{eq}}$ and $\rho_{2}^{\mathrm{eq}}$ has a similar behavior. Even though it is cheaper to compute $\eta_{2}^{\text {eq }}$ than $\eta_{1}^{\text {eq }}$, the effectivities of the two strategies are really close.

Finally, comparing the effectivites $\rho_{2}^{\text {eq }}$ with $\hat{\rho}$ it is clear that the new strategy to compute the linear equilibrated tractions in the edges of the mesh is much more competitive versus the standard one, although the computational cost is not significantly increased.

Regarding the inequalities introduced in (41) and (42), note that only for the flux-free approach, $\rho^{q} \leq \rho^{\text {st }}$ in the meshes of 32,128 and 512 elements. In all the other cases, the inequalities hold as expected.

Figure 4 shows the elements most contributing to the squared norm of the error for the final mesh of 32768 elements. Specifically, the elements $\Omega_{k} \subset \mathcal{T}_{75 \%}$ are such that

$$
\sum_{\Omega_{k} \subset \mathcal{T}_{75 \%}}\|e\|_{k}^{2} \geq 0.75\|\mid\|^{2}
$$

and $\mathcal{T}_{75 \%}$ is such that it verifies the previous inequality with the minimum number of elements. These elements represent the $38 \%$ of the elements of the mesh and as can be seen are concentrated around the four corners of the domain.
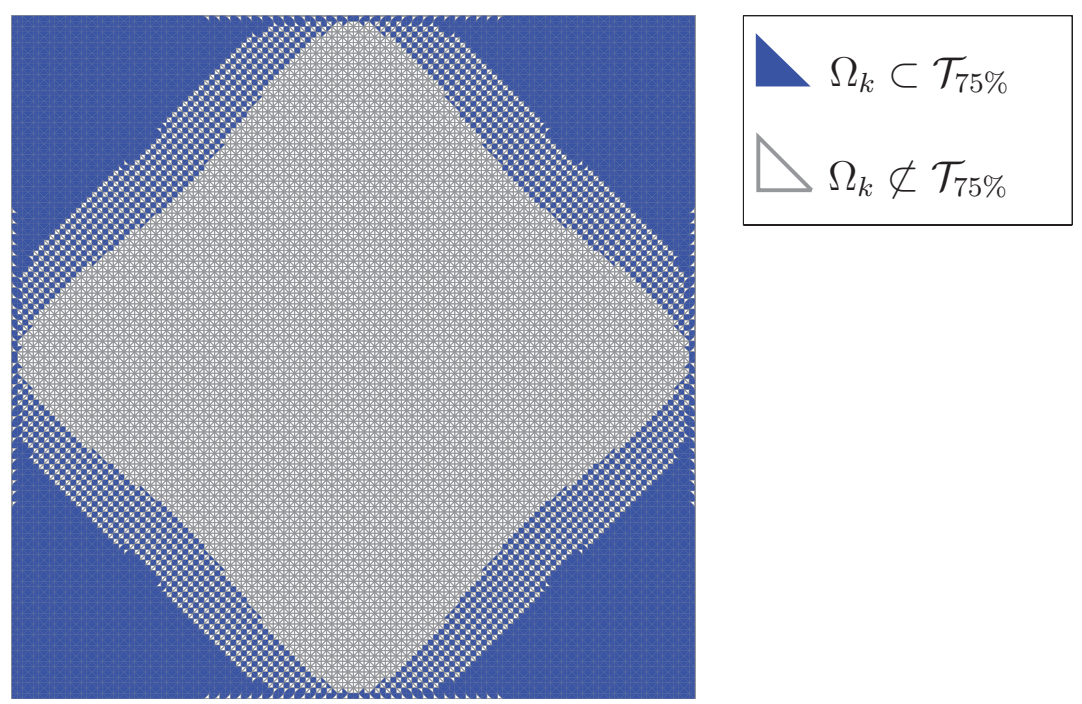

Figure 4: Example 1: Elements with larger squared error contributions for the final mesh of 32768 elements. The sum of the local squared norm of the error for the selected elements reaches the $75 \%$ of the total squared error.

For this mesh, the local squared effectivity indices $\eta_{k}^{2} /\|e\|_{k}^{2}, \hat{\eta}_{k}^{2} /\|e\|_{k}^{2}$ and $\left(\eta_{2}^{\mathrm{eq}}\right)_{k}^{2} /\|e\|_{k}^{2}$ are also considered. The histograms in Figure 5 represent the occurrences of the local squared effectivity indices 
for these three estimates. The histograms show the number of elements with local effectivity in a
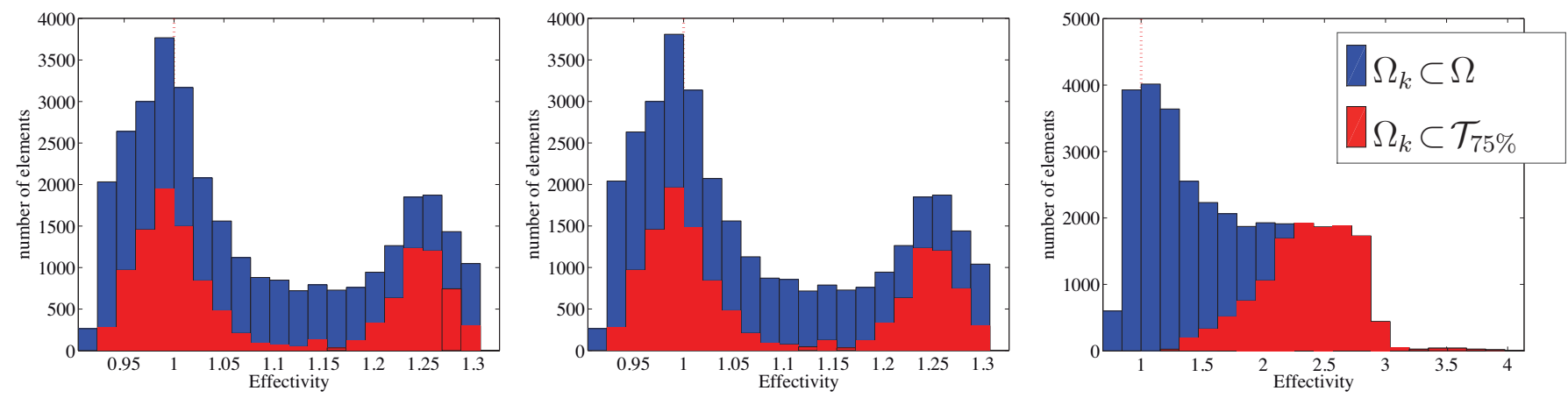

Figure 5: Example 1: Histograms for local squared effectivity indices for the upper bounds $\eta$ (left), $\hat{\eta}$ (middle) and $\eta_{2}^{\text {eq }}$ (right). The effectivities of the elements $\Omega_{k} \subset \mathcal{T}_{75 \%}$ are highlighted in the figures.

given range. The histograms show a good behavior of the estimates if they display a narrow distribution (all elements have similar local effectivity indices) concentrated around 1. As can be seen, the new flux-free equilibration strategies provides much better effectivities than the estimate obtained using the standard equilibration procedure since the local effectivity indices are much more closer to one. Observe that the local values associated with the new flux-free equilibration estimates $\eta$ and $\hat{\eta}$ proposed here are much more accurate than the values corresponding to the standard equilibration procedure $\eta_{2}^{\text {eq }}$. The histogram also highlights the effectivities of the elements in $\mathcal{T}_{75 \%}$.

The ranges of the local non-squared effectivities of the whole mesh and in the elements in $\mathcal{T}_{75 \%}$ are shown in Table 4. As can be seen, the standard equilibration technique yields a considerable

\begin{tabular}{cc|ccc}
\hline & & $\eta$ & $\hat{\eta}$ & $\eta_{2}^{\text {eq }}$ \\
\hline $\min$ & $\eta_{k}^{*} /\|e\|_{k}$ & 0.9631 & 0.9631 & 1.1252 \\
$\max$ & $\eta_{k}^{*} /\|e \mid\|_{k}$ & 1.1401 & 1.1401 & 1.9881 \\
\hline
\end{tabular}

Table 4: Example 1: ranges for the local effectivity indices of the elements in $\mathcal{T}_{75 \%}$.

overestimation on the relevant elements while the local effectivities in the new equilibration technique are much better. Thus, not only the global upper bound provides better results, but also the local indicators are more accurate and therefore better suited for adaptivity.

\subsection{L-shaped domain with analytical solution}

In this example the Poisson equation is considered in a non-convex domain, with $f=0$. The domain is the standard L-shaped domain with a reentrant corner $\Omega=(-1,1) \times(0,1) \cup(-1,0) \times(-1,0)$. The boundary conditions are Dirichlet homogenous on all the boundary, that is, $\Gamma_{\mathrm{D}}=\partial \Omega$, and the source data $f$ is chosen so that the exact solution to the problem is

$$
u(r, \theta)=\left(1-r^{2} \cos ^{2}(\theta)\right)\left(1-r^{2} \sin ^{2}(\theta)\right)\left(r^{2 / 3}-r^{3}\right) \sin (2 \theta / 3),
$$

where $r$ is the distance from the reentrant corner point and $\theta$ is the angle from the upper surface of the corner $[0,1] \times\{0\}$.

The behavior of the bounds is both analyzed for a uniform and an adaptive mesh refinement. In the adaptive procedure, the mesh is adaptively refined using a bulk criterion [8]. In particular, the set of elements marked for refinement is the one which has the smallest number of elements verifying that the sum of the contributions toward the estimator from these elements exceeds $50 \%$ of the value of the estimator. Both in the uniform and adaptive refinements, each element of the mesh is subdivided into two new ones by bisecting the longest edge of each specified triangle. 


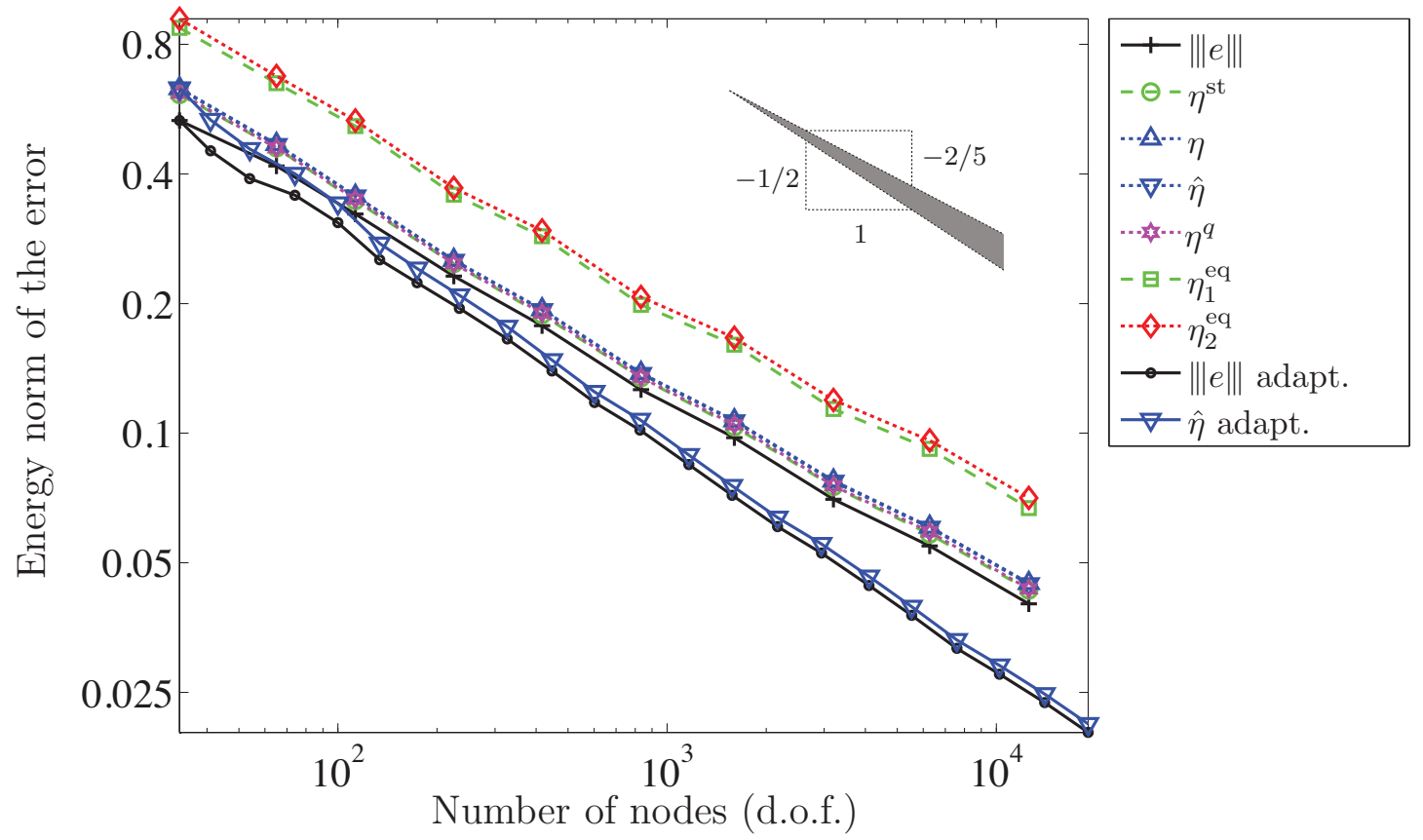

Figure 6: Example 2: Comparison of the convergence of the bounds using either a uniform $h$-refinement or using an adaptive $h$-refinement strategy. The results for the adaptive procedure are only shown for the estimate $\hat{\eta}$ (appearing in the legend of the figure with the adapt. tag).

Figure 6 shows the upper bounds provided by all the estimates when a uniform mesh refinement is performed, along with the corresponding exact energy norm. For this problem, the obtained convergence of the bounds when a uniform mesh refinement is considered is approximately $\mathcal{O}\left(h^{2 / 5}\right)$ or equivalently $\mathcal{O}\left(n_{\mathrm{np}}^{-1 / 5}\right)$. As in the previous example, the estimates associated to the flux-free strategies provide more accurate results than the ones obtained using the standard equilibrated strategies. It is also confirmed that the cheapest estimates $\eta, \hat{\eta}$ and $\eta_{2}^{\text {eq }}$ provide very good results when compared to its associated more expensive estimates $\eta^{\text {st }}$ and $\eta_{1}^{\text {eq }}$ respectively. Moreover, the estimates $\eta$ and $\hat{\eta}$ provide practically identical results.

Regarding the adaptive procedure, the same relation between the estimates is observed, and therefore only the results for the estimate $\hat{\eta}$ are shown. As can be seen, the accuracy of the estimate $\hat{\eta}$ is very good when compared to the exact energy norm of the error. It is worth noting that Figure 6 contains two values for the exact energy norm, one obtained using the meshes of the uniform refinement procedure, and the other one obtained using the adapted meshes. Therefore, the uniform estimates have to be compared to the line with legend $\||\|\mid\|$ whereas the adaptive estimate has to be compared with the error named in the legend after $\|e \mid\|$ adapt.

Figure 7 shows the effectivity indices of the estimators analyzed in Figure 6 . It illustrates that the new equilibration strategy provides very competitive results when compared with the standard equilibration procedure that clearly overestimates the exact error. Note that however, all the estimates provide guaranteed upper bounds for the error since all the obtained effectivities are larger that one. A sample of the sequence of meshes obtained in the adaptive procedure is shown in Figure 8. The estimates for the error and its effectivity indexes for these selected meshes are shown in Table 5. Again it can be seen that the results are very accurate. Also, for the mesh of 1575 nodes, Figure 9 shows the elements which belong to $\Omega_{k} \subset \mathcal{T}_{50 \%}$. Those 902 elements represent $30 \%$ of the elements of the mesh and are the ones that should be refined if the exact error was used to mark the elements for refinement. This figure also shows the elements marked for refinement if the estimates $\hat{\eta}$ and $\eta_{2}^{\text {eq }}$ are used as local indicators. In this case the number of elements marked for refinement are 922 and 692 elements respectively, representing the $31 \%$ and $23 \%$ of the total elements. Both estimates provide good indicators for the adaptive procedure, but the one using the standard equilibrated tractions 


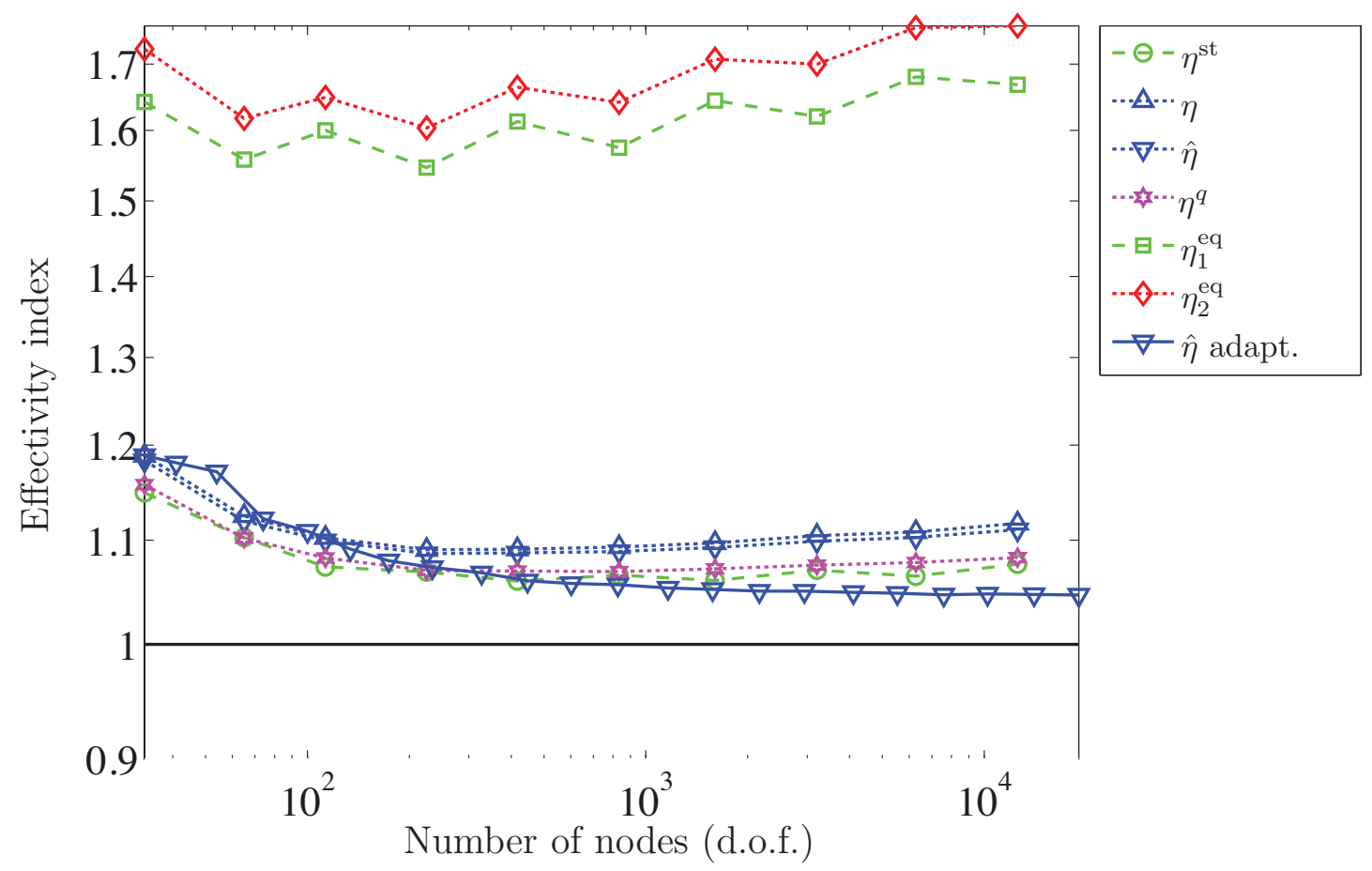

Figure 7: Example 2: Effectivity indices obtained in the uniform an adaptive $h$-refinement strategies. The results for the adaptive procedure are only shown for the estimate $\hat{\eta}$ (appearing in the legend of the figure with the adapt. tag).
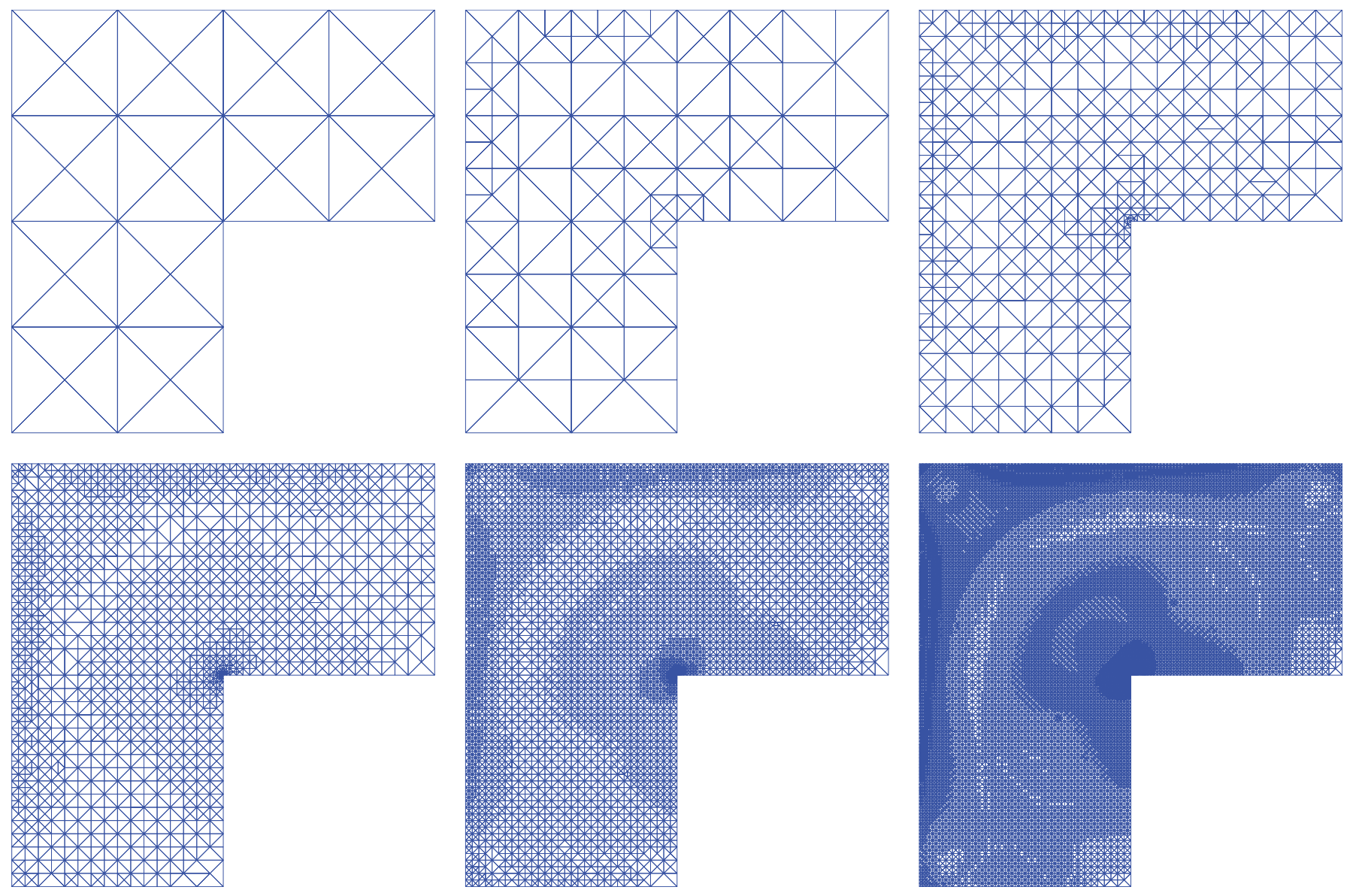

Figure 8: Example 2: Sample of the sequence of meshes obtained with the adaptive $h$-refinement strategy. The meshes correspond to iterations 1,5,10,14, 18 and 22 with 33, 100, 447, 1575, 5535 and 19030 nodes respectively. 


\begin{tabular}{c||cc||cc}
\hline$n_{\mathrm{np}}$ & $\left\|u_{h}\right\| \|$ & $\|e \mid\|$ & $\hat{\eta}$ & $\hat{\rho}$ \\
\hline 33 & 0.82701 & 0.53210 & 0.62945 & 1.18297 \\
100 & 0.93390 & 0.30808 & 0.34055 & 1.10542 \\
447 & 0.97346 & 0.13948 & 0.14767 & 1.05874 \\
1575 & 0.98078 & 0.07174 & 0.07539 & 1.05096 \\
5535 & 0.98268 & 0.03773 & 0.03953 & 1.04761 \\
19030 & 0.98319 & 0.02019 & 0.02111 & 1.04601 \\
\hline
\end{tabular}

Table 5: Example 2: results for some of the steps of the adaptive procedure. The sample steps are chosen so that the error from one iteration to the other are nearly divided by two.
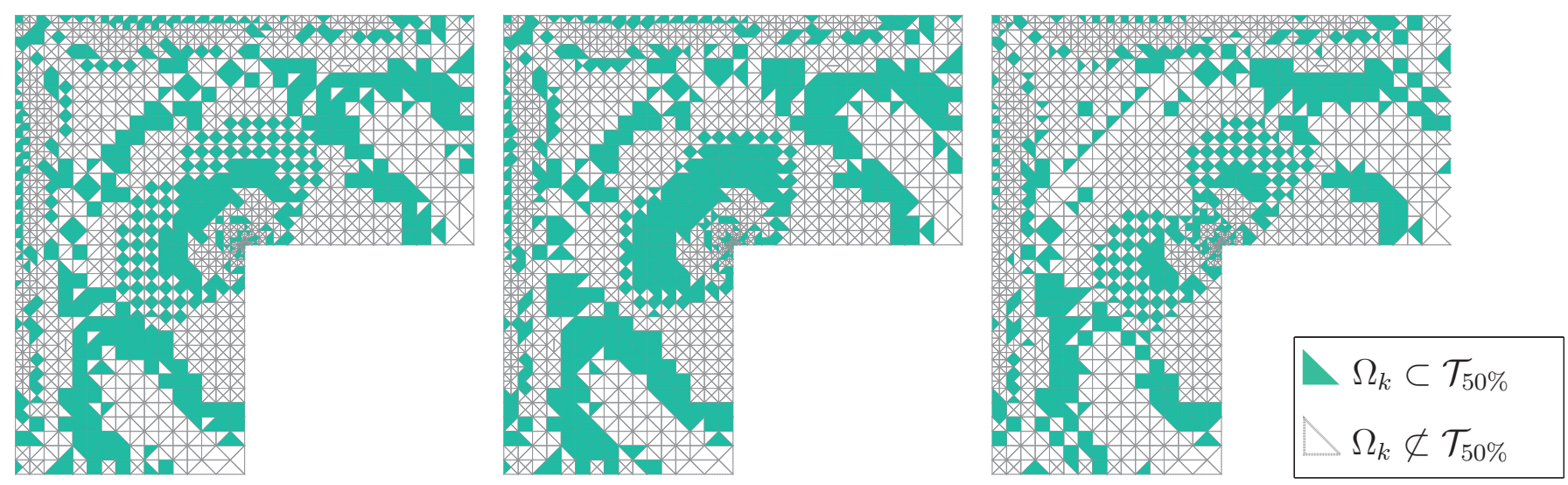

Figure 9: Example 2: Elements with larger squared error contributions for the intermediate mesh of 2972 elements. The sum of the local squared norm of the exact error for the selected elements reaches the $50 \%$ of the total squared exact error (left). Elements reaching the $50 \%$ of the error estimate are shown for $\hat{\eta}$ and $\eta_{2}^{\text {eq }}$ on the middle and right respectively.

instead of refining around $30 \%$ of the elements in each adaptive step as one would do if the exact error was used as indicator, only refines around $20-25 \%$ of the elements. This is due to the fact that the standard equilibrated tractions provide a larger overestimation of the error, which can be seen in Figure 10. In this figure, the local squared effectivity indices $\hat{\eta}_{k}^{2} /\|e\|_{k}^{2}$ and $\left(\eta_{2}^{\mathrm{eq}}\right)_{k}^{2} /\|e\|_{k}^{2}$ are
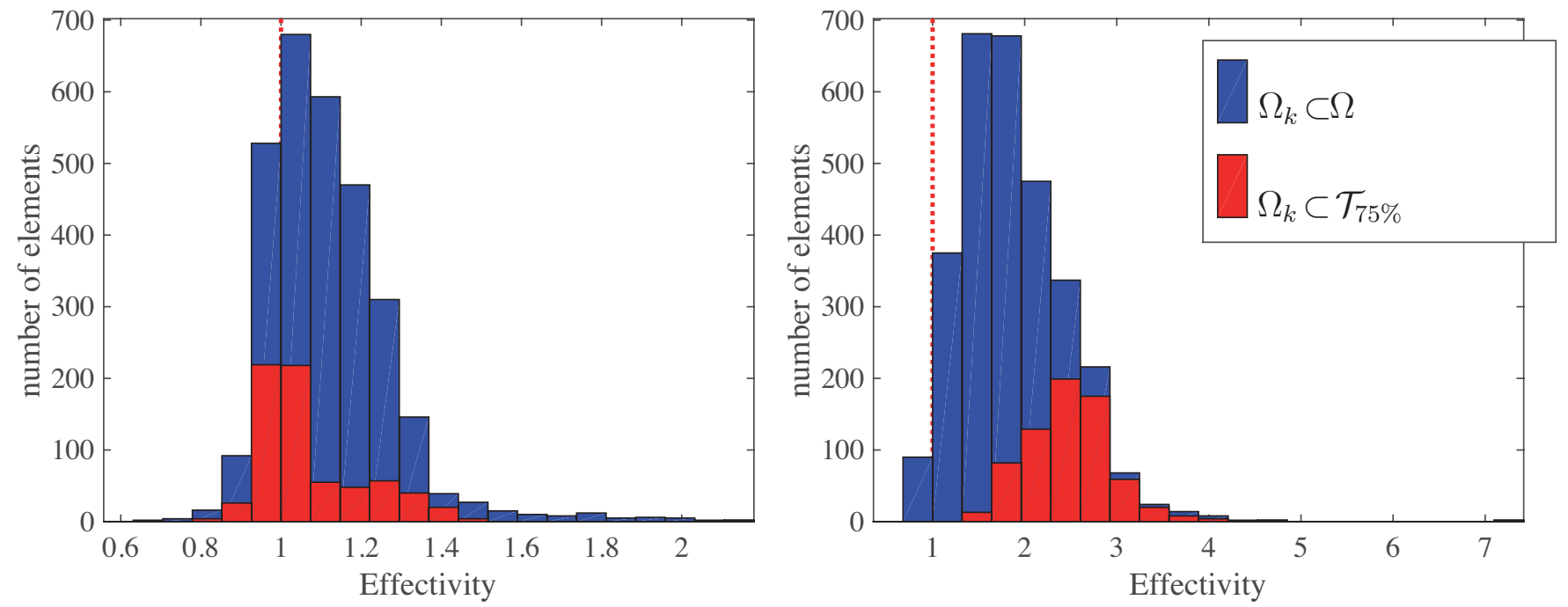

Figure 10: Example 2: Histograms for local squared effectivity indices for the upper bounds $\hat{\eta}$ (left) and $\eta_{2}^{\text {eq }}$ (right) for the mesh of 1575 nodes. The effectivities of the elements $\Omega_{k}, k \in \mathcal{T}_{50 \%}$ are highlighted in the figures.

shown. The histograms represent the occurrences of the local squared effectivity indices for these 
estimates, highlighting the effectivities of the elements $\Omega_{k}, k \in \mathcal{T}_{50 \%}$.

\subsection{Reaction-diffusion problem with interior layer}

The reaction-diffusion equation ( 1 ) is considered in the square $\Omega=(-1,1)^{2}$, where the right-hand side $f$ is such that the exact solution of the problem is

$$
u=1-\tanh \left(\kappa\left(x^{2}+y^{2}-\frac{1}{4}\right)\right) .
$$

The boundary conditions are all Dirichlet, that is, $\Gamma_{\mathrm{D}}=\partial \Omega$. Note that, even though in the present results the Dirichlet boundary conditions are linearly interpolated in each mesh, for large enough values of $\kappa, u$ is nearly equal to zero on the boundary, and therefore, the Dirichlet boundary conditions can be considered to be homogeneous which can be exactly imposed with a linear interpolation. Thus, it can be considered that there is no data error due to the interpolation of the Dirichlet boundary conditions, and therefore the upper bounds are going to be guaranteed upper bounds for the exact error. The exact solution of this problem exhibits an interior layer along the boundary of the circle of radius $1 / 2$ centered at the origin. The exact solution for three different values of $\kappa$ are shown in Figure (11).
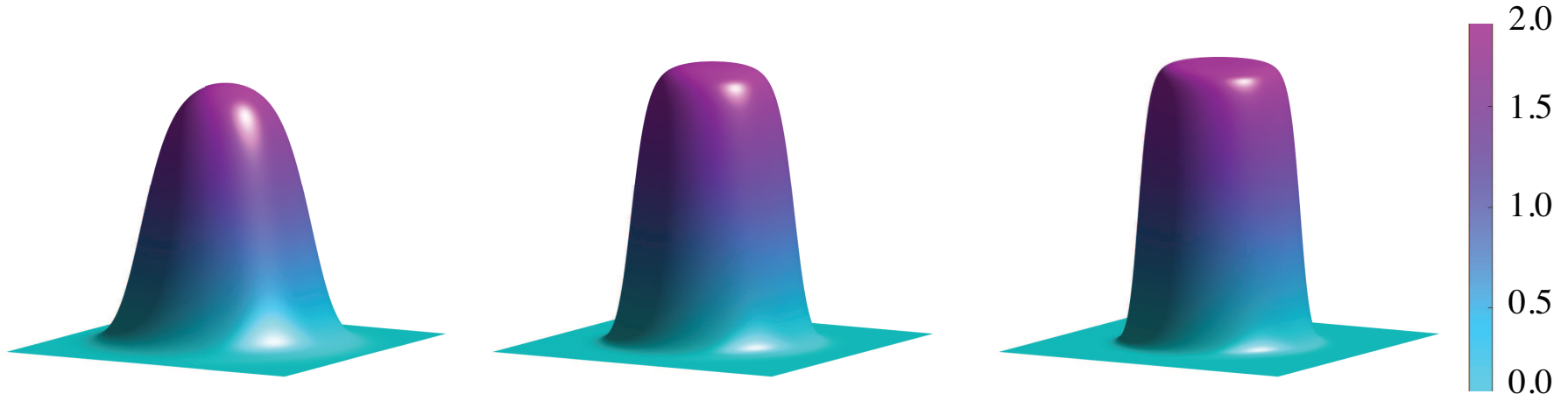

Figure 11: Example 3: Exact solution of the problem for the values $\kappa=5$ (left), $\kappa=10$ (middle) and $\kappa=15$ (right).

The obtained error estimates and effectivities for the adaptive procedure are shown in Figure 12. The adaptive meshes are obtained using the local indicator associated to $\hat{\eta}$, in particular, the set of elements marked for refinement is the one which has the smallest number of elements verifying that the sum of the contributions toward the estimator from these elements exceeded $50 \%$ of the value of the estimator. For these meshes, the estimates $\eta_{\mathbf{s t}}$ and $\eta_{2}^{\text {eq }}$ are also computed to compare the effectivities of the estimates.

Although the robustness of the presented estimates in the singular regime for large values of $\kappa$ is not discussed here, it can be seen that as $\kappa$ grows, the effectivities for the initial meshes are worst, but that after a few adaptive steps very good effectivities are obtained. For the singularly-perturbed reaction-diffusion problem, the robustness of the bounds is not guaranteed for the strategies presented in this work, and will be a matter of discussion in a forthcoming paper.

Figure 13 shows the final meshes obtained in the adaptive procedure of 18639, 11573 and 10719 nodes respectively. The adaptive procedure is stopped when the error estimate $\hat{\eta}$ is reduced by a factor of around 1/100 with respect to the initial error estimate. As can be seen, in the three cases, the local indicators allow properly refining the areas where the interior layer forms.

The results obtained for the initial, intermediate and final meshes are shown in Table 6. The intermediate mesh is selected so that the error estimate $\hat{\eta}$ is reduced by a factor of around 1/10 with respect to its initial value. 

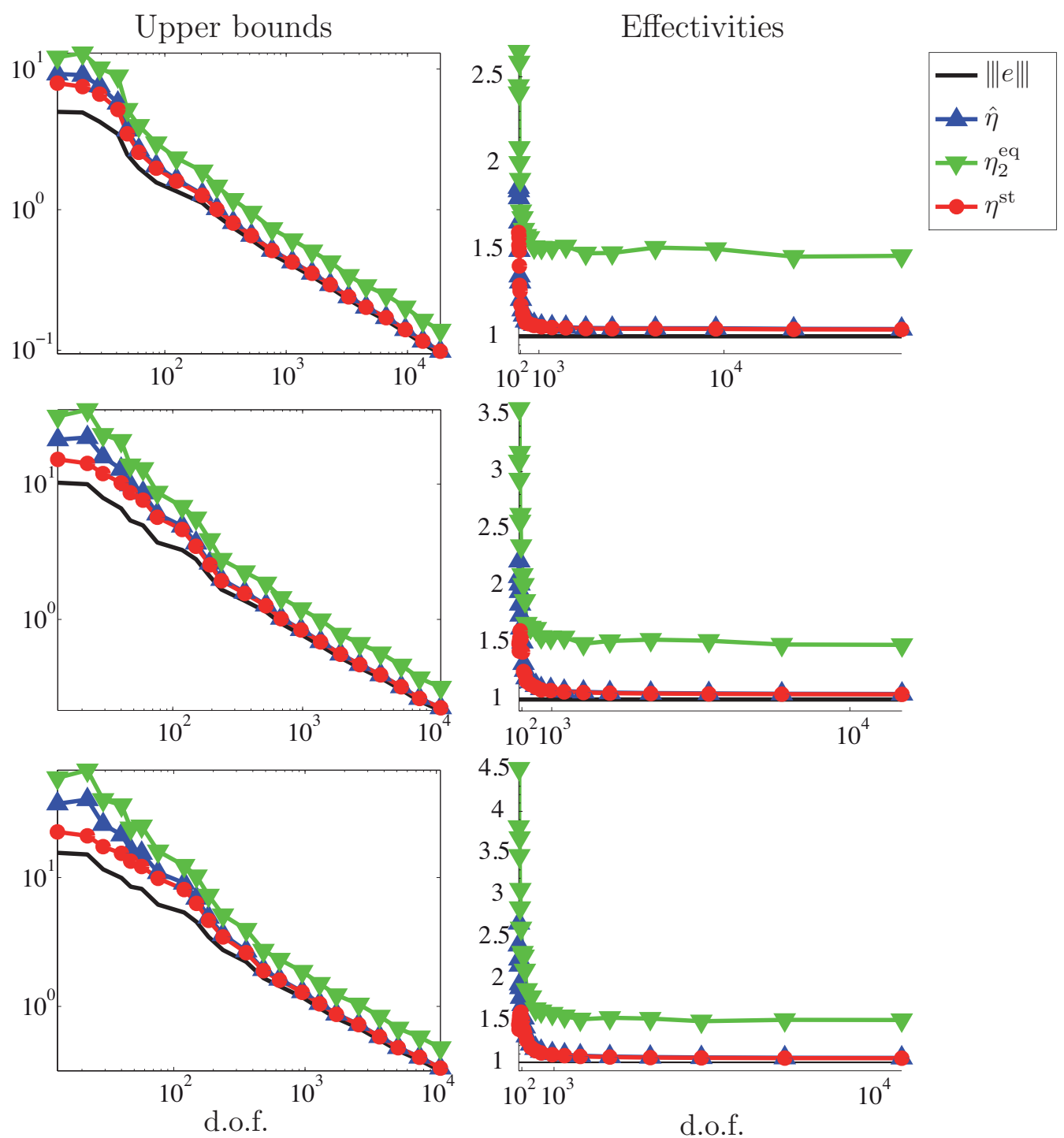

Figure 12: Example 3: Estimates and effectivites for the adaptive procedure for $\kappa=5$ (top), $\kappa=10$ (middle) and $\kappa=15$ (down).
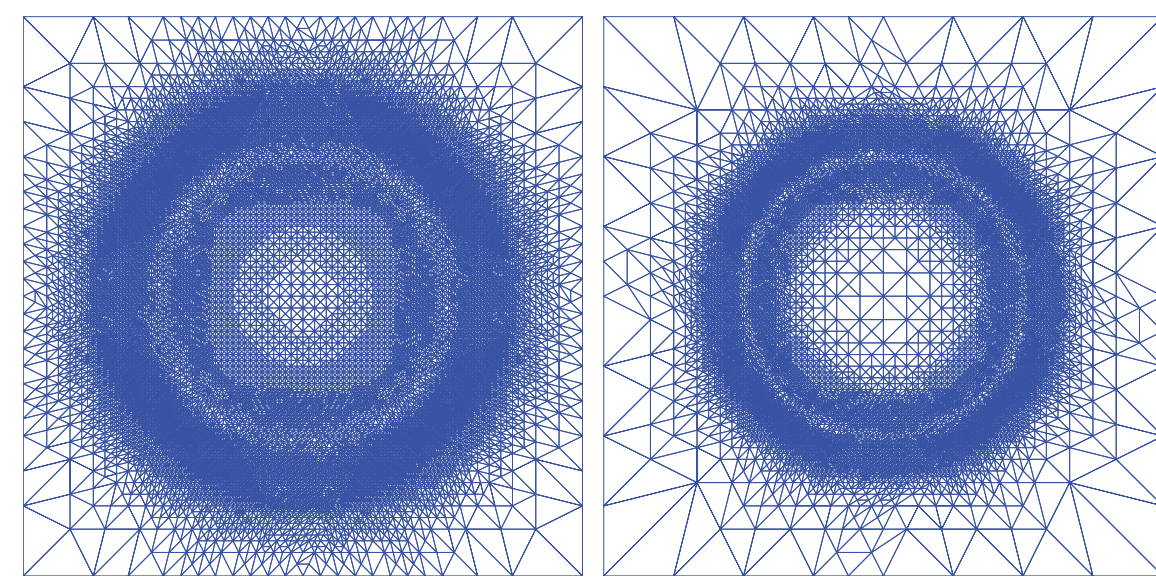

Figure 13: Example 3: Final meshes $\kappa=5$ (left), $\kappa=10$ (middle) and $\kappa=15$ (right). The meshes have 18639, 11573 and 10719 nodes respectively

\section{Concluding remarks}

A new technique to compute guaranteed upper bounds for the energy norm of the error of twodimensional finite element piecewise linear approximations of the exact solution has been introduced. 


\begin{tabular}{|c|c|c|c|c|c|c|c|c|}
\hline & & & \multicolumn{3}{|c|}{ bounds } & \multicolumn{3}{|c|}{ effectivities } \\
\hline$n_{\mathrm{np}}$ & $\left|u_{h}\right|$ & $\mid e \|$ & $\eta^{\mathrm{st}}$ & $\hat{\eta}$ & $\eta_{2}^{\mathrm{eq}}$ & $\rho^{\mathrm{st}}$ & $\hat{\rho}$ & $\rho_{2}^{\mathrm{eq}}$ \\
\hline 13 & 6.95890 & 4.95748 & 7.92989 & 9.22971 & 12.13324 & 1.56 & 1.86 & 2.45 \\
\hline ॥ 366 & 8.51168 & 0.74446 & 0.80291 & 0.81039 & 1.17814 & 1.08 & 1.09 & 1.58 \\
\hline 난 18639 & 8.54366 & 0.09449 & 0.09821 & 0.09850 & 0.13842 & 1.04 & 1.04 & 1.47 \\
\hline 13 & 13.75572 & 10.23907 & 15.23529 & 21.29163 & 31.64774 & 1.49 & 2.08 & 3.09 \\
\hline ㅍ 237 & 17.06746 & 1.66137 & 1.92728 & 1.97799 & 2.77015 & 1.16 & 1.19 & 1.67 \\
\hline ㄴ 11573 & 17.14681 & 0.21254 & 0.22202 & 0.22282 & 0.31442 & 1.04 & 1.05 & 1.48 \\
\hline $\begin{array}{ll}\stackrel{2}{\rightarrow} & 13\end{array}$ & 20.81366 & 15.56722 & 22.59131 & 37.41622 & 59.37906 & 1.45 & 2.40 & 3.81 \\
\hline ॥ 237 & 25.84622 & 2.74216 & 3.45869 & 3.61280 & 5.12175 & 1.26 & 1.32 & 1.87 \\
\hline 갈 10719 & 25.98935 & 0.31648 & 0.33196 & 0.33346 & 0.47666 & 1.05 & 1.05 & 1.51 \\
\hline
\end{tabular}

Table 6: Example 3: bounds and effectivities in a series of adaptively $h$-refined linear triangular meshes.

The bounds are guaranteed regardless of the size of the underlying finite element mesh and regardless of the kind of data (the source term and the Neumann boundary conditions are not required to be piecewise polynomial functions).

The proposed strategy may be seen as either: (1) an improved cheap version of the flux-free technique presented in $[20,18]$ or (2) a new more efficient hybrid-flux equilibrated residual method.

A deep analysis of the new approach reveals that alleviating the cost of the flux-free approach does not introduce a significant difference on the accuracy of the results. The original upper bounds are only slightly lower than the new upper bounds. It is also confirmed that the equilibrated tractions provided by the new approach yield much sharper bounds than the original equilibrated strategies. Therefore, the proposed strategy is clearly competitive to obtain guaranteed upper bounds for the error (both in accuracy and cost).

Some open issues will be addressed in future investigations, namely (1) extension to higher-dimensional problems and (2) analysis of the robustness of the approach when dealing with singularly perturbed reaction-diffusion equations.

\section{Acknowledgements}

This work was supported by the Ministerio de Economía y Competitividad [DPI2014-51844-C2-2-R].

\section{Appendix A. Proof of Theorem 1}

Lemma 1. Let $\Omega_{k}$ be a triangle and denote by $h_{k}$ its diameter. Let $\kappa$ be a non-negative constant. Then, for any $v \in \mathcal{H}^{1}\left(\Omega_{k}\right)$

$$
\begin{aligned}
& \|v\|_{k} \leq \frac{1}{\kappa}\|v\|_{k} \quad \text { for } \kappa>0, \\
& \|\nabla v\|_{k} \leq\|v\|_{k} \\
& \left\|v-\bar{v}_{k}\right\|_{k} \leq \frac{h_{k}}{\pi}\|\nabla v\|_{k},
\end{aligned}
$$

where $\bar{v}_{k}$ denotes the average value of $v$ in element $\Omega_{k}$, that is $\bar{v}_{k}=\left(\int_{\Omega_{k}} v d \Omega\right) /\left|\Omega_{k}\right|$, and $\|v \mid\|_{k}$ is the restriction of the energy norm $\|v\|=a(v, v)^{1 / 2}$ to element $\Omega_{k}$.

Note that equations (A.1a) and (A.1b) are directly obtained noting that for a constant reaction coefficient $\kappa,\|v\|_{k}^{2}=\|\nabla v\|_{k}^{2}+\kappa^{2}\|v\|_{k}^{2}$, while (A.1c) is the well-known Poincaré inequality [3]. 
Lemma 2. Let $\Omega_{k}$ be a triangle and let $\gamma$ be one of its edges. Let $h_{k}$ be the diameter of the triangle and $\kappa$ a non-negative constant. Then, for any $v \in \mathcal{H}^{1}\left(\Omega_{k}\right)$

$$
\begin{aligned}
& \|v\|_{\gamma} \leq C_{1}\|v\|_{k} \quad \text { for } \kappa>0 \\
& \left\|v-\bar{v}_{\gamma}\right\|_{\gamma} \leq C_{2}\|v\|_{k}
\end{aligned}
$$

where $\bar{v}_{\gamma}$ is the average value of $v$ on edge $\gamma, \bar{v}_{\gamma}=\left(\int_{\gamma} v d \Gamma\right) /|\gamma|$, and the constants $C_{1}$ and $C_{2}$ are given in equations (11) and (12) or (14) respectively.

Proof The inequalities presented in this Lemma are similar to the trace inequalities given in [3] for the particular case $d=2$ and the proof is analogous. Let's start with the first trace inequality given in $(\mathrm{A} .2 \mathrm{a})$.

Let $\boldsymbol{x}_{\gamma}$ be the vertex of $\Omega_{k}$ opposite to the edge $\gamma$ and consider the function $\boldsymbol{\varphi}(\boldsymbol{x})=\boldsymbol{x}-\boldsymbol{x}_{\gamma}$ for all $\boldsymbol{x} \in \Omega_{k}$. It is easy to see that $\boldsymbol{\varphi}(\boldsymbol{x}) \cdot \boldsymbol{n}=0$ on $\Omega_{k} \backslash \gamma$ and that $\boldsymbol{\varphi}(\boldsymbol{x}) \cdot \boldsymbol{n}=2\left|\Omega_{k}\right| /|\gamma|$ on $\gamma$, where $\boldsymbol{n}$ is the outward unit normal to $\partial \Omega_{k}$. Note that the altitude of the triangle with respect to $\gamma$ coincides with $2\left|\Omega_{k}\right| /|\gamma|$.

Consider now a function $v \in \mathcal{H}^{1}\left(\Omega_{k}\right)$, then from the definition of the $\mathcal{L}^{2}(\gamma)$-norm and using that the value of $\boldsymbol{\varphi}(\boldsymbol{x}) \cdot \boldsymbol{n}$ is known in the three edges of the triangle $\left(2\left|\Omega_{k}\right| /|\gamma|\right.$ on $\gamma$ and zero elsewhere) yields

$$
\frac{2\left|\Omega_{k}\right|}{|\gamma|}\|v\|_{\gamma}^{2}=\frac{2\left|\Omega_{k}\right|}{|\gamma|} \int_{\gamma} v^{2} d \Gamma=\int_{\gamma} v^{2} \boldsymbol{\varphi} \cdot \boldsymbol{n} d \Gamma=\int_{\partial \Omega_{k}} v^{2} \boldsymbol{\varphi} \cdot \boldsymbol{n} d \Gamma,
$$

which can be rewritten using the divergence theorem and the fact that $\nabla \cdot \varphi=2$ as

$$
\begin{aligned}
\int_{\partial \Omega_{k}} v^{2} \boldsymbol{\varphi} \cdot \boldsymbol{n} d \Gamma & =\int_{\Omega_{k}} \boldsymbol{\nabla} \cdot\left(v^{2} \boldsymbol{\varphi}\right) d \Omega=\int_{\Omega_{k}}\left(\boldsymbol{\nabla}\left(v^{2}\right) \cdot \boldsymbol{\varphi}+v^{2} \boldsymbol{\nabla} \cdot \boldsymbol{\varphi}\right) d \Omega \\
& =2 \int_{\Omega_{k}}\left(v \boldsymbol{\varphi} \cdot \boldsymbol{\nabla} v+v^{2}\right) d \Omega .
\end{aligned}
$$

Joining equations (A.3) and (A.4) and using the definition of the $\mathcal{L}^{2}\left(\Omega_{k}\right)$-norm along with the CauchySchwarz inequality reveals that

$$
\frac{2\left|\Omega_{k}\right|}{|\gamma|}\|v\|_{\gamma}^{2}=2 \int_{\Omega_{k}}(v \boldsymbol{\varphi} \cdot \nabla v) d \Omega+2 \int_{\Omega_{k}} v^{2} d \Omega \leq 2\|v \boldsymbol{\varphi}\|_{k}\|\nabla v\|_{k}+2\|v\|_{k}^{2} .
$$

Moreover, denoting by $|\boldsymbol{\varphi}|$ the Euclidean norm in $\mathbb{R}^{2}$ the first term of the previous equation may be bounded by

$$
\|v \boldsymbol{\varphi}\|_{k}^{2}=\int_{\Omega_{k}} v^{2} \boldsymbol{\varphi}^{\top} \boldsymbol{\varphi} d \Omega=\int_{\Omega_{k}} v^{2}|\boldsymbol{\varphi}|^{2} d \Omega \leq\left(\max _{\boldsymbol{x} \in \Omega_{k}}|\boldsymbol{\varphi}|\right)^{2} \int_{\Omega_{k}} v^{2} d \Omega=\left(\max _{\boldsymbol{x} \in \gamma}|\boldsymbol{\varphi}|\right)^{2}\|v\|_{k}^{2} .
$$

Therefore

$$
\frac{2\left|\Omega_{k}\right|}{|\gamma|}\|v\|_{\gamma}^{2} \leq 2\|v\|_{k}\left(\left(\max _{\boldsymbol{x} \in \gamma}|\varphi|\right)\|\nabla v\|_{k}+\|v\|_{k}\right)
$$

Now, for $\kappa>0$, noting that the previous expression can be rewritten as a scalar $\mathbb{R}^{2}$ product and 
using the Cauchy-Schwarz inequality in $\mathbb{R}^{2}$ yields

$$
\begin{aligned}
\frac{2\left|\Omega_{k}\right|}{|\gamma|}\|v\|_{\gamma}^{2} & =2\|v\|_{k}\left\langle\left(\max _{\boldsymbol{x} \in \gamma}|\boldsymbol{\varphi}|, \frac{1}{\kappa}\right),\left(\|\boldsymbol{\nabla} v\|_{k}, \kappa\|v\|_{k}\right)\right\rangle \\
& \leq 2\|v\|_{k}\left(\left(\max _{\boldsymbol{x} \in \gamma}|\boldsymbol{\varphi}|\right)^{2}+\frac{1}{\kappa^{2}}\right)^{1 / 2}\left(\|\boldsymbol{\nabla} v\|_{k}^{2}+\kappa\|v\|_{k}^{2}\right)^{1 / 2} \\
& =2\left(\left(\max _{\boldsymbol{x} \in \gamma}|\boldsymbol{\varphi}|\right)^{2}+\frac{1}{\kappa^{2}}\right)^{1 / 2}\|v\|_{k}\|v\|_{k} .
\end{aligned}
$$

Since $\kappa>0$, inequality (A.1a) can be used to finally obtain

$$
\frac{2\left|\Omega_{k}\right|}{|\gamma|}\|v\|_{\gamma}^{2} \leq \frac{2}{\kappa} \sqrt{\left(\max _{\boldsymbol{x} \in \gamma}\left|\boldsymbol{x}-\boldsymbol{x}_{\gamma}\right|\right)^{2}+\frac{1}{\kappa^{2}}}\|v\|_{k}^{2},
$$

where $\varphi$ has been replaced by its definition.

The first trace inequality (A.2a) is then obtained by simply taking square roots of the previous equation and isolating $\|v\|_{\gamma}$.

Let's now move to the second trace inequality (A.2b). Note that by definition

$$
\left\|v-\bar{v}_{\gamma}\right\|_{\gamma} \leq\|v-c\|_{\gamma}
$$

for any real constant $c \in \mathbb{R}$. In particular, taking $c=\bar{v}_{k}$ in the previous inequality, that is, taking $c$ equal to the average value of $v$ in element $\Omega_{k}$, and applying equation (A.5) to the function $v-\bar{v}_{k}$ yields

$$
\left\|v-\bar{v}_{\gamma}\right\|_{\gamma}^{2} \leq\left\|v-\bar{v}_{k}\right\|_{\gamma}^{2} \leq \frac{|\gamma|}{\left|\Omega_{k}\right|}\left\|v-\bar{v}_{k}\right\|_{k}\left(\left(\max _{\boldsymbol{x} \in \gamma}|\varphi|\right)\|\nabla v\|_{k}+\left\|v-\bar{v}_{k}\right\|_{k}\right) .
$$

Let's now observe that for $\kappa>0$

$$
\left\|v-\bar{v}_{k}\right\|_{k} \leq C_{0}\|v\|_{k}
$$

where recall that $C_{0}=\min \left\{h_{k} / \pi, 1 / \kappa\right\}$. Indeed, using the Poincaré inequality (A.1c) along with (A.1b) it holds that for any value of $\kappa \geq 0$

$$
\left\|v-\bar{v}_{k}\right\|_{k} \leq \frac{h_{k}}{\pi}\|\nabla v\|_{k} \leq \frac{h_{k}}{\pi}\|v\|_{k}
$$

and for $\kappa>0$ using the definition of $\bar{v}_{k}$, which ensures an analogous inequality to (A.6), and inequality (A.1a) it also holds that

$$
\left\|v-\bar{v}_{k}\right\|_{k} \leq\|v\|_{k} \leq \frac{1}{\kappa}\|v\|_{k} .
$$

Thus, joining these two inequalities equation (A.8) follows. Note that for $\kappa=0$, the minimum appearing in the definition of $C_{0}$ should be replaced by $h_{k} / \pi$ since only the Poincaré inequality can be used. Hereafter, to join the cases $\kappa>0$ and $\kappa=0$ into a single formula, the following abuse of notation $C_{0}=\min \left\{h_{k} / \pi, 1 / \kappa\right\}=h_{k} / \pi$ is used for $\kappa=0$. Note that using this notation, the definition of the parameters $C_{0}$ and $C_{2}$ given in (10) and(12) turns out into (13) and (14) for $\kappa=0$. Inequality (A.2b) is then obtained by using inequalities (A.1b) and (A.8) into (A.7).

$$
\left\|v-\bar{v}_{\gamma}\right\|_{\gamma}^{2} \leq \frac{|\gamma|}{\left|\Omega_{k}\right|} C_{0}\left(\left(\max _{\boldsymbol{x} \in \gamma}\left|\boldsymbol{x}-\boldsymbol{x}_{\gamma}\right|\right)+C_{0}\right)\|v\|_{k}^{2}
$$

where again $\varphi$ has been replaced by its definition.

Proof of Theorem 1. Let $\boldsymbol{q} \in\left[\mathcal{L}^{2}(\Omega)\right]^{2}$ and $r \in \mathcal{L}^{2}(\Omega)$ and denote by $\boldsymbol{q}_{k}$ and $r_{k}$ its restrictions to 
element $\Omega_{k}$, that is $\boldsymbol{q}_{k}=\left.\boldsymbol{q}\right|_{\Omega_{k}}$ and $r_{k}=\left.r\right|_{\Omega_{k}}$ respectively. Then

$$
\int_{\Omega}\left(\boldsymbol{q} \cdot \boldsymbol{\nabla} v+\kappa^{2} r v\right) d \Omega=\sum_{k=1}^{n_{\mathrm{el}}} \int_{\Omega_{k}}\left(\boldsymbol{q}_{k} \cdot \nabla v+\kappa^{2} r_{k} v\right) d \Omega
$$

and using equations (3) and (8) and the definition of $\ell^{\Pi}(\cdot)$ it is clear that

$$
\begin{aligned}
& a(e, v)=\ell(v)-a\left(u_{h}, v\right) \\
& =\ell^{\Pi}(v)-a\left(u_{h}, v\right)+\sum_{k=1}^{n_{\mathrm{el}}}\left[\int_{\Omega_{k}}\left(f-\Pi_{k}^{\hat{q}} f\right) v d \Omega+\sum_{\gamma \subset \Gamma_{\mathrm{N}} \cap \partial \Omega_{k}} \int_{\gamma}\left(g_{\mathrm{N}}-\Pi_{\gamma}^{\bar{q}} g_{\mathrm{N}}\right) v d \Gamma\right] \\
& \quad=\sum_{k=1}^{n_{\mathrm{el}}}\left[\int_{\Omega_{k}}\left(\boldsymbol{q}_{k} \cdot \nabla v+\kappa^{2} r_{k} v\right) d \Omega+\int_{\Omega_{k}}\left(f-\Pi_{k}^{\hat{q}} f\right) v d \Omega+\sum_{\gamma \subset \Gamma_{\mathrm{N}} \cap \partial \Omega_{k}} \int_{\gamma}\left(g_{\mathrm{N}}-\Pi_{\gamma}^{\bar{q}} g_{\mathrm{N}}\right) v d \Gamma\right] .
\end{aligned}
$$

The term containing the source data oscillation can be bounded using property (6) along with an application of the Cauchy-Schwarz inequality and (A.8) as

$$
\begin{aligned}
\int_{\Omega_{k}}\left(f-\Pi_{k}^{\hat{q}} f\right) v d \Omega & =\int_{\Omega_{k}}\left(f-\Pi_{k}^{\hat{q}} f\right)\left(v-\bar{v}_{k}\right) d \Omega \\
& \leq\left\|f-\Pi_{k}^{\hat{q}} f\right\|_{k}\left\|v-\bar{v}_{k}\right\|_{k} \leq C_{0}\left\|f-\Pi_{k}^{\hat{q}} f\right\|_{k}\|v\|_{k} .
\end{aligned}
$$

Similarly, using the Cauchy-Schwarz inequality along with inequalities (A.2a) and (A.2b) and property (7), the Neumann data oscillation can be both bounded by

$$
\int_{\gamma}\left(g_{\mathrm{N}}-\Pi_{\gamma}^{\bar{q}} g_{\mathrm{N}}\right) v d \Gamma \leq\left\|g_{\mathrm{N}}-\Pi_{\gamma}^{\bar{q}} g_{\mathrm{N}}\right\|\left\|_{\gamma}\right\| v\left\|_{\gamma} \leq C_{1}\right\| g_{\mathrm{N}}-\Pi_{\gamma}^{\bar{q}} g_{\mathrm{N}}\|\|_{\gamma}\|v\|_{k} \quad \text { for } \kappa>0
$$

and

$$
\int_{\gamma}\left(g_{\mathrm{N}}-\Pi_{\gamma}^{\bar{q}} g_{\mathrm{N}}\right)\left(v-\bar{v}_{\gamma}\right) d \Gamma \leq\left\|g_{\mathrm{N}}-\Pi_{\gamma}^{\bar{q}} g_{\mathrm{N}}\right\|_{\gamma}\left\|v-\bar{v}_{\gamma}\right\|_{\gamma} \leq C_{2}\left\|g_{\mathrm{N}}-\Pi_{\gamma}^{\bar{q}} g_{\mathrm{N}}\right\|_{\gamma}\|v\|_{k}
$$

Therefore, the Neumann term can be bounded by

$$
\int_{\gamma}\left(g_{\mathrm{N}}-\Pi_{\gamma}^{\bar{q}} g_{\mathrm{N}}\right) v d \Gamma \leq \min \left\{C_{1}, C_{2}\right\}\left\|g_{\mathrm{N}}-\Pi_{\gamma}^{\bar{q}} g_{\mathrm{N}}\right\|_{\gamma}\|v\|_{k}
$$

where for $\kappa=0$, the minimum $\min \left\{C_{1}, C_{2}\right\}$ has to be replaced by $C_{2}$.

Introducing the bounds (A.10) and (A.11) into (A.9) and applying the Cauchy-Schwarz inequality to the first term results in

$$
\begin{aligned}
& a(e, v)= \sum_{k=1}^{n_{\mathrm{el}}}\left[\int_{\Omega_{k}}\left(\boldsymbol{q}_{k} \cdot \nabla v+\kappa^{2} r_{k} v\right) d \Omega+\int_{\Omega_{k}}\left(f-\Pi_{k}^{\hat{q}} f\right) v d \Omega+\sum_{\gamma \subset \Gamma_{\mathrm{N}} \cap \partial \Omega_{k}} \int_{\gamma}\left(g_{\mathrm{N}}-\Pi_{\gamma}^{\bar{q}} g_{\mathrm{N}}\right) v d \Gamma\right] \\
& \leq \sum_{k=1}^{n_{\mathrm{el}}}\left[\sqrt{\|\boldsymbol{q}\|_{k}^{2}+\kappa^{2}\|r\|_{k}^{2}}\|v\|_{k}+C_{0}\left\|f-\Pi_{k}^{\hat{q}} f\right\|_{k}\|v\|_{k}\right. \\
&\left.\quad+\sum_{\gamma \subset \Gamma_{\mathrm{N}} \cap \partial \Omega_{k}} \min \left\{C_{1}, C_{2}\right\}\left\|g_{\mathrm{N}}-\Pi_{\gamma}^{\bar{q}} g_{\mathrm{N}}\right\| \gamma\|v\|_{k}\right] \\
& \leq \sum_{k=1}^{n_{\mathrm{el}}}\left[\sqrt{\|\boldsymbol{q}\|_{k}^{2}+\kappa^{2}\|r\|_{k}^{2}}+\operatorname{osc}_{k}(f)+\sum_{\gamma \subset \Gamma_{\mathrm{N}} \cap \partial \Omega_{k}} \operatorname{osc}_{\gamma}\left(g_{\mathrm{N}}\right)\right]\|v\|_{k}=\sum_{k=1}^{n_{\mathrm{el}}} \eta_{k}\|v\|_{k} .
\end{aligned}
$$


Finally, the Cauchy-Schwarz inequality in $\mathbb{R}^{n_{\mathrm{el}}}$ and the definition of the energy norm is used to yield

$$
a(e, v) \leq \sqrt{\sum_{k=1}^{n_{\mathrm{el}}} \eta_{k}^{2}} \sqrt{\sum_{k=1}^{n_{\mathrm{el}}}\|v\|_{k}^{2}}=\sqrt{\sum_{k=1}^{n_{\mathrm{el}}} \eta_{k}^{2}}\|v\|
$$

The proof of the theorem concludes substituting $v=e$ in the previous inequality, noting that $a(e, e)=\|e\|^{2}$ and squaring the final inequality.

\section{Appendix B. Strong version of (16)}

Lemma 3. Let $\left\{\boldsymbol{q}_{k}^{i}, r_{k}^{i}\right\}_{\Omega_{k} \subset \omega_{i}}$ be a set of dual estimates verifying equation (18) for a given set of tractions $\left\{g_{\gamma}^{i}\right\}_{\gamma \subset \omega_{i}}$ verifying (17). Then, the global star dual estimates $\boldsymbol{q}^{i}$ and $r^{i}$ such that $\left.\boldsymbol{q}^{i}\right|_{\Omega_{k}}=\boldsymbol{q}_{k}^{i}$ and $\left.r^{i}\right|_{\Omega_{k}}=r_{k}^{i}$ verify equation (16) with $\hat{q}=1$ and $\bar{q}=0$.

Proof Let's first simplify the term $a\left(u_{h}, \phi_{i} v\right)$ in the r.h.s. of equation (16). Using the definition of the bilinear form $a(\cdot, \cdot)$, the divergence theorem and that for linear elements $\boldsymbol{\nabla} u_{h} \cdot \boldsymbol{\nabla} \phi_{i} v+\nabla u_{h} \cdot \nabla v \phi_{i}=$ $\boldsymbol{\nabla} \cdot\left(\phi_{i} \nabla u_{h} v\right)$ yields

$$
\begin{aligned}
a\left(u_{h}, \phi_{i} v\right) & =\int_{\omega_{i}}\left(\nabla u_{h} \cdot \nabla\left(\phi_{i} v\right)+\kappa^{2} u_{h} \phi_{i} v\right) d \Omega \\
& =\int_{\omega_{i}}\left(\nabla u_{h} \cdot \nabla \phi_{i} v+\nabla u_{h} \cdot \nabla v \phi_{i}+\kappa^{2} u_{h} \phi_{i} v\right) d \Omega \\
& =\int_{\omega_{i}}\left(\nabla \cdot\left(\phi_{i} \nabla u_{h} v\right)+\kappa^{2} u_{h} \phi_{i} v\right) d \Omega \\
& =\int_{\omega_{i}} \kappa^{2} u_{h} \phi_{i} v d \Omega+\sum_{\Omega_{k} \subset \omega_{i}} \sum_{\gamma \subset \partial \Omega_{k}} \int_{\gamma} \phi_{i} \nabla u_{h} \cdot \boldsymbol{n}_{k}^{\gamma} v d \Gamma .
\end{aligned}
$$

Now, separating the integral over the star appearing in the l.h.s. of (16) into elemental integrals, applying the divergence theorem, using equation (18) along with the fact that for linear elements $\boldsymbol{\nabla} \cdot\left(\boldsymbol{\nabla} u_{h}\right)=0$, and finally using (B.1) yields

$$
\begin{aligned}
& \int_{\omega_{i}}\left(\boldsymbol{q}^{i} \cdot \nabla v+\kappa^{2} r^{i} v\right) d \Omega=\sum_{\Omega_{k} \subset \omega_{i}} \int_{\Omega_{k}}\left(\boldsymbol{q}_{k}^{i} \cdot \nabla v+\kappa^{2} r_{k}^{i} v\right) d \Omega \\
& =\sum_{\Omega_{k} \subset \omega_{i}} \int_{\Omega_{k}}\left(-\nabla \cdot \boldsymbol{q}_{k}^{i}+\kappa^{2} r_{k}^{i}\right) v d \Omega+\sum_{\Omega_{k} \subset \omega_{i}} \sum_{\gamma \subset \partial \Omega_{k}} \int_{\gamma} \boldsymbol{q}_{k}^{i} \cdot \boldsymbol{n}_{k}^{\gamma} v d \Gamma \\
& =\sum_{\Omega_{k} \subset \omega_{i}} \int_{\Omega_{k}}\left(\phi_{i} \Pi_{k}^{1} f-\kappa^{2} u_{h} \phi_{i}-\nabla u_{h} \cdot \nabla \phi_{i}+\nabla \cdot\left(\phi_{i} \nabla u_{h}\right)\right) v d \Omega \\
& +\sum_{\Omega_{k} \subset \omega_{i}} \sum_{\gamma \subset \partial \Omega_{k}} \int_{\gamma}\left(\sigma_{k}^{\gamma} g_{\gamma}^{i}-\phi_{i} \nabla u_{h} \cdot \boldsymbol{n}_{k}^{\gamma}\right) v d \Gamma \\
& =\sum_{\Omega_{k} \subset \omega_{i}} \int_{\Omega_{k}}\left(\phi_{i} \Pi_{k}^{1} f-\kappa^{2} u_{h} \phi_{i}\right) v d \Omega+\sum_{\Omega_{k} \subset \omega_{i}} \sum_{\gamma \subset \partial \Omega_{k}} \int_{\gamma}\left(\sigma_{k}^{\gamma} g_{\gamma}^{i}-\phi_{i} \nabla u_{h} \cdot \boldsymbol{n}_{k}^{\gamma}\right) v d \Gamma \\
& =\sum_{\Omega_{k} \subset \omega_{i}} \int_{\Omega_{k}} \Pi_{k}^{1} f \phi_{i} v d \Omega+\sum_{\Omega_{k} \subset \omega_{i}} \sum_{\gamma \subset \partial \Omega_{k}} \int_{\gamma} \sigma_{k}^{\gamma} g_{\gamma}^{i} v d \Gamma-a\left(u_{h}, \phi_{i} v\right) .
\end{aligned}
$$

The summation over the edges of the elements in the previous equation can be separated into boundary and interior edges as

$$
\sum_{\Omega_{k} \subset \omega_{i}} \sum_{\gamma \subset \partial \Omega_{k}} \int_{\gamma} \sigma_{k}^{\gamma} g_{\gamma}^{i} v d \Gamma=\sum_{\gamma \subset \partial \omega_{i} \cap \Gamma_{\mathrm{N}}} \int_{\gamma} g_{\gamma}^{i} v d \Gamma+\sum_{\gamma=\partial \Omega_{k} \cap \partial \Omega_{k^{\prime}} \subset \omega_{i}} \int_{\gamma}\left(\sigma_{k}^{\gamma}+\sigma_{k^{\prime}}^{\gamma}\right) g_{\gamma}^{i} v d \Gamma .
$$


It is worth noting that the edges $\gamma \subset \partial \omega_{i} \cap \Gamma_{\mathrm{D}}$ have not been included in the last equation because in these Dirichlet edges the test function $v \in \mathcal{V}\left(\omega_{i}\right)$ vanishes. The same applies for the edges $\gamma \subset \partial \omega_{i} \backslash \partial \Omega \subset \mathcal{Z}_{i}$, since the tractions $g_{\gamma}^{i}$ have a zero value in $\mathcal{Z}_{i}$, see (17). Then, since $\sigma_{k}^{\gamma}+\sigma_{k^{\prime}}^{\gamma}=0$, the summation over the edges reduces to the Neumann edges of the boundary of the domain, and using equation (17b) yields

$$
\sum_{\Omega_{k} \subset \omega_{i}} \sum_{\gamma \subset \partial \Omega_{k}} \int_{\gamma} \sigma_{k}^{\gamma} g_{\gamma}^{i} v d \Gamma=\sum_{\gamma \subset \partial \omega_{i} \cap \Gamma_{\mathrm{N}}} \int_{\gamma} g_{\gamma}^{i} v d \Gamma=\sum_{\gamma \subset \partial \omega_{i} \cap \Gamma_{\mathrm{N}}} \int_{\gamma} \phi_{i} \Pi_{\gamma}^{0} g_{\mathrm{N}} v d \Gamma .
$$

Therefore, for all $v \in \mathcal{V}\left(\omega_{i}\right)$

$$
\begin{aligned}
\int_{\omega_{i}}\left(\boldsymbol{q}^{i} \cdot \boldsymbol{\nabla}\right. & \left.v+\kappa^{2} r^{i} v\right) d \Omega \\
& =\sum_{\Omega_{k} \subset \omega_{i}} \int_{\Omega_{k}} \Pi_{k}^{1} f \phi_{i} v d \Omega+\sum_{\gamma \subset \partial \omega_{i} \cap \Gamma_{\mathrm{N}}} \int_{\gamma} \phi_{i} \Pi_{\gamma}^{0} g_{\mathrm{N}} v d \Gamma-a\left(u_{h}, \phi_{i} v\right) \\
& =\ell^{\Pi}\left(\phi_{i} v\right)-a\left(u_{h}, \phi_{i} v\right)
\end{aligned}
$$

and the proof is concluded.

\section{Appendix C. Closed expression for the local weighted flux $q_{k}^{i}$}

Theorem 3. Let $\omega_{i}$ be the star of the mesh associated to node $i$ and let $r_{k}^{i}$ and $\boldsymbol{q}_{k}^{i}$ be the weighted dual estimates defined by equations (21), (22), (23) and (24). Then, for any choice of the local tractions $g_{\gamma}^{i}$ verifying (17) and the weighted equilibration condition (20) or its equivalent form (27), $r_{k}^{i}$ and $\boldsymbol{q}_{k}^{i}$ verify equation (18).

Proof Note that since $r_{k}^{i}=0$, equation (18) can be simplified to

$$
\begin{array}{rrr}
-\nabla \cdot\left(\boldsymbol{q}_{k}^{i}+\phi_{i} \boldsymbol{\nabla} u_{h}\right) & =\phi_{i}\left(\Pi_{k}^{1} f-\kappa^{2} u_{h}\right)-\boldsymbol{\nabla} u_{h} \cdot \nabla \phi_{i} & \text { in } \Omega_{k} \\
\left(\boldsymbol{q}_{k}^{i}+\phi_{i} \boldsymbol{\nabla} u_{h}\right) \cdot \boldsymbol{n}_{k}^{\gamma} & =\sigma_{k}^{\gamma} g_{\gamma}^{i} & \text { on } \gamma \subset \partial \Omega_{k} .
\end{array}
$$

Now, consider first equation (C.1b) on edge $\gamma_{[1]}$. On this edge, since $\left.\lambda_{[1]}\right|_{\gamma_{[1]}}=0$, the normal component of the linear flux defined in (23) can be simplified to

$$
\mathbf{q}_{k}^{i L} \cdot \boldsymbol{n}_{[1]}=\frac{1}{2\left|\Omega_{k}\right|}\left(\boldsymbol{\rho}_{[2]}^{k} \cdot \boldsymbol{n}_{[1]} \lambda_{[2]}+\boldsymbol{\rho}_{[3]}^{k} \cdot \boldsymbol{n}_{[1]} \lambda_{[3]}\right),
$$

where recall that $\boldsymbol{n}_{[1]}=\boldsymbol{n}_{k}^{\gamma[1]}$. Moreover, taking into account that elementary algebra reveals that

$$
l_{[1]} \boldsymbol{t}_{[3]}^{\top} \boldsymbol{n}_{[1]}=2\left|\Omega_{k}\right| \quad, \quad l_{[1]} \boldsymbol{t}_{[2]}^{\top} \boldsymbol{n}_{[1]}=-2\left|\Omega_{k}\right|
$$

yields

$$
\begin{aligned}
& \boldsymbol{\rho}_{[2]}^{k} \cdot \boldsymbol{n}_{[1]}=\left.2\left|\Omega_{k}\right|\left(\sigma_{k}^{\gamma} g_{\gamma}^{i}-\phi_{i} \boldsymbol{\nabla} u_{h} \cdot \boldsymbol{n}_{k}\right)\right|_{\gamma_{[1]}}\left(\boldsymbol{x}_{[2]}\right) \\
& \boldsymbol{\rho}_{[3]}^{k} \cdot \boldsymbol{n}_{[1]}=\left.2\left|\Omega_{k}\right|\left(\sigma_{k}^{\gamma} g_{\gamma}^{i}-\phi_{i} \boldsymbol{\nabla} u_{h} \cdot \boldsymbol{n}_{k}\right)\right|_{\gamma_{[1]}}\left(\boldsymbol{x}_{[3]}\right)
\end{aligned}
$$

and therefore

$$
\begin{aligned}
\mathbf{q}_{k}^{i L} \cdot \boldsymbol{n}_{[1]} & =\left.\left(\sigma_{k}^{\gamma} g_{\gamma}^{i}-\phi_{i} \boldsymbol{\nabla} u_{h} \cdot \boldsymbol{n}_{k}\right)\right|_{\gamma_{[1]}}\left(\boldsymbol{x}_{[2]}\right) \lambda_{[2]}+\left.\left(\sigma_{k}^{\gamma} g_{\gamma}^{i}-\phi_{i} \boldsymbol{\nabla} u_{h} \cdot \boldsymbol{n}_{k}\right)\right|_{\gamma_{[1]}}\left(\boldsymbol{x}_{[3]}\right) \lambda_{[3]} \\
& =\sigma_{k}^{\gamma} g_{\gamma_{[1]}^{i}}^{i}-\phi_{i} \boldsymbol{\nabla} u_{h} \cdot \boldsymbol{n}_{[1]}
\end{aligned}
$$

since both $g_{\gamma_{[1]}^{i}}^{i}$ and $\phi_{i} \boldsymbol{\nabla} u_{h} \cdot \boldsymbol{n}_{[1]}$ are linear functions over $\gamma_{[1]}$. 
Also note that $\mathbf{q}_{k}^{i C}$ has vanishing normal components on each edge of $\Omega_{k}$. Indeed, consider edge $\gamma_{[1]}$. Then, noting that $\left.\beta_{[2]}\right|_{\gamma_{[1]}}=\left.\beta_{[3]}\right|_{\gamma_{[1]}}=0$ and that $\boldsymbol{t}_{[1]} \cdot \boldsymbol{n}_{[1]}=0$ yields

$$
\mathbf{q}_{k}^{i C} \cdot \boldsymbol{n}_{[1]}=\boldsymbol{n}_{[1]}^{\top} \mathbf{q}_{k}^{i C}=\frac{1}{3}\left(\beta_{[1]} \boldsymbol{n}_{[1]}^{\top} \boldsymbol{t}_{[1]} \boldsymbol{t}_{[1]}^{\top}+\beta_{[2]} \boldsymbol{n}_{[1]}^{\top} \boldsymbol{t}_{[2]} \boldsymbol{t}_{[2]}^{\top}+\beta_{[3]} \boldsymbol{n}_{[1]}^{\top} \boldsymbol{t}_{[3]} \boldsymbol{t}_{[3]}^{\top}\right) \nabla v^{Q}=0 .
$$

Analogous arguments apply to the other two edges, and we conclude that

$$
\mathbf{q}_{k}^{i} \cdot \boldsymbol{n}_{k}^{\gamma}=\mathbf{q}_{k}^{i L} \cdot \boldsymbol{n}_{k}^{\gamma}+\mathbf{q}_{k}^{i C} \cdot \boldsymbol{n}_{k}^{\gamma}=\sigma_{k}^{\gamma} g_{\gamma}^{i}-\phi_{i} \boldsymbol{\nabla} u_{h} \cdot \boldsymbol{n}_{k}^{\gamma}
$$

and equation (C.1b) is verified.

Let's move now to the divergence condition (C.1a). Observe that both $\boldsymbol{q}_{k}^{i L}$ and $\phi_{i} \boldsymbol{\nabla} u_{h}$ are linear and therefore they have constant divergence over $\Omega_{k}$. Thus,

$$
\nabla \cdot\left(\boldsymbol{q}_{k}^{i L}+\phi_{i} \boldsymbol{\nabla} u_{h}\right)=\frac{1}{\left|\Omega_{k}\right|} \int_{\Omega_{k}} \nabla \cdot\left(\boldsymbol{q}_{k}^{i L}+\phi_{i} \nabla u_{h}\right) d \Omega
$$

Now, using the divergence theorem along with the fact that $\mathbf{q}_{k}^{i C}$ has vanishing normal components on each edge of $\Omega_{k}$, yields

$$
\nabla \cdot\left(\boldsymbol{q}_{k}^{i L}+\phi_{i} \boldsymbol{\nabla} u_{h}\right)=\frac{1}{\left|\Omega_{k}\right|} \int_{\partial \Omega_{k}}\left(\boldsymbol{q}_{k}^{i L}+\phi_{i} \boldsymbol{\nabla} u_{h}\right) \cdot \boldsymbol{n}_{k} d \Gamma=\frac{1}{\left|\Omega_{k}\right|} \int_{\partial \Omega_{k}}\left(\boldsymbol{q}_{k}^{i}+\phi_{i} \boldsymbol{\nabla} u_{h}\right) \cdot \boldsymbol{n}_{k} d \Gamma .
$$

Finally, inserting (C.1b) in the previous equation, and using the fact that, since $\boldsymbol{x}_{[1]}$ is the central node $\gamma_{[1]} \in \mathcal{Z}_{i}, g_{\gamma_{[1]}}^{i}=0$ from (17a), after some algebraic manipulations it follows that

$$
\begin{aligned}
& \nabla \cdot\left(\boldsymbol{q}_{k}^{i L}+\phi_{i} \nabla u_{h}\right)=\frac{1}{\left|\Omega_{k}\right|} \sum_{\gamma \subset \partial \Omega_{k}} \int_{\gamma} \sigma_{k}^{\gamma} g_{\gamma}^{i} d \Gamma \\
& =\frac{l_{[2]}}{2\left|\Omega_{k}\right|}\left(\sigma_{k}^{\gamma_{[2]}} \alpha_{21}+\sigma_{k}^{\gamma_{[2]}} \alpha_{23}\right)+\frac{l_{[3]}}{2\left|\Omega_{k}\right|}\left(\sigma_{k}^{\gamma_{[3]}} \alpha_{31}+\sigma_{k}^{\gamma_{[3]}} \alpha_{32}\right),
\end{aligned}
$$

where the notation for the weighted tractions $g_{\gamma}^{i}$ in element $\Omega_{k}$ from star $\omega_{i}$ introduced in Figure 2 is used.

In order to compute the divergence of the cubic contribution to the flux $\mathbf{q}_{k}^{i C}$, first the basic divergence property $\nabla \cdot(c \boldsymbol{v})=c \nabla \cdot \boldsymbol{v}+\boldsymbol{v} \cdot \nabla c$, along with the following relations

$$
\nabla \cdot\left(\beta_{[1]} \boldsymbol{t}_{[1]}\right)=\lambda_{[2]}-\lambda_{[3]}, \nabla \cdot\left(\beta_{[2]} \boldsymbol{t}_{[2]}\right)=\lambda_{[3]}-\lambda_{[1]}, \nabla \cdot\left(\beta_{[3]} \boldsymbol{t}_{[3]}\right)=\lambda_{[1]}-\lambda_{[2]}
$$

are used to yield, after rearranging terms,

$$
\begin{aligned}
\nabla \cdot \mathbf{q}_{k}^{i C} & =\frac{1}{3} \nabla \cdot\left(\left(\beta_{[1]} \boldsymbol{t}_{[1]}\right)\left(\boldsymbol{t}_{[1]}^{\top} \nabla v^{Q}\right)+\left(\beta_{[2]} \boldsymbol{t}_{[2]}\right)\left(\boldsymbol{t}_{[2]}^{\top} \nabla v^{Q}\right)+\left(\beta_{[3]} \boldsymbol{t}_{[3]}\right)\left(\boldsymbol{t}_{[3]}^{\top} \nabla v^{Q}\right)\right) \\
& =\frac{1}{3}\left(\lambda_{[1]}\left(\boldsymbol{t}_{[3]}^{\top}-\boldsymbol{t}_{[2]}^{\top}\right)+\lambda_{[2]}\left(\boldsymbol{t}_{[1]}^{\top}-\boldsymbol{t}_{[3]}^{\top}\right)+\lambda_{[3]}\left(\boldsymbol{t}_{[2]}^{\top}-\boldsymbol{t}_{[1]}^{\top}\right)\right) \nabla v^{Q} \\
& +\frac{1}{3}\left(\beta_{[1]} \boldsymbol{t}_{[1]}\right) \cdot \nabla\left(\boldsymbol{t}_{[1]}^{\top} \nabla v^{Q}\right)+\frac{1}{3}\left(\beta_{[2]} \boldsymbol{t}_{[2]}\right) \cdot \nabla\left(\boldsymbol{t}_{[2]}^{\top} \nabla v^{Q}\right)+\frac{1}{3}\left(\beta_{[3]} \boldsymbol{t}_{[3]}\right) \cdot \nabla\left(\boldsymbol{t}_{[3]}^{\top} \nabla v^{Q}\right) .
\end{aligned}
$$

Noting now that $\nabla \lambda_{[i]}=-\frac{l_{[i]}}{2\left|\Omega_{k}\right|} \boldsymbol{n}_{[i]}$, the expression for the gradient of $v^{Q}$, see equation (25), can be 
simplified to

$$
\begin{aligned}
\nabla v^{Q} & =-\frac{1}{16\left|\Omega_{k}\right|}\left(l_{[1]}\left(3 F+3 F_{[1]} \lambda_{[1]}+4 F_{[1]}\right) \boldsymbol{n}_{[1]}\right. \\
& \left.+l_{[2]}\left(3 F_{[2]} \lambda_{[1]}-F_{[3]}\right) \boldsymbol{n}_{[2]}+l_{[3]}\left(3 F_{[3]} \lambda_{[1]}-F_{[2]}\right) \boldsymbol{n}_{[3]}\right) .
\end{aligned}
$$

Moreover, using the following relations

$$
l_{[i]} \boldsymbol{n}_{[i]} \cdot \boldsymbol{t}_{[i j]}=2\left|\Omega_{k}\right| \quad, \quad l_{[i]} \boldsymbol{n}_{[i]} \cdot \boldsymbol{t}_{[j i]}=-2\left|\Omega_{k}\right|
$$

where the new auxiliary notation for the tangent vectors has to be considered $\boldsymbol{t}_{[23]}=\boldsymbol{t}_{[1]}, \boldsymbol{t}_{[31]}=\boldsymbol{t}_{[2]}$ and $\boldsymbol{t}_{[12]}=\boldsymbol{t}_{[3]}$, yields after simplifying again

$$
\begin{aligned}
\boldsymbol{t}_{[1]}^{\top} \nabla v^{Q} & =-\frac{1}{8}\left(3 \lambda_{[1]}+1\right)\left(F_{[2]}-F_{[3]}\right) \\
\boldsymbol{t}_{[2]}^{\top} \nabla v^{Q} & =\frac{1}{8}\left(F_{[1]}\left(6 \lambda_{[1]}+4\right)+F_{[2]}\left(3 \lambda_{[2]}+1\right)+3 F_{[3]}\left(\lambda_{[3]}-\lambda_{[1]}\right)\right) \\
\boldsymbol{t}_{[3]}^{\top} \nabla v^{Q} & =-\frac{1}{8}\left(F_{[1]}\left(6 \lambda_{[1]}+4\right)+3 F_{[2]}\left(\lambda_{[2]}-\lambda_{[1]}\right)+F_{[3]}\left(3 \lambda_{[3]}+1\right)\right)
\end{aligned}
$$

and

$$
\begin{aligned}
& \nabla\left(\boldsymbol{t}_{[1]}^{\top} \nabla v^{Q}\right)=\frac{3\left(F_{[2]}-F_{[3]}\right) l_{[1]}}{16\left|\Omega_{k}\right|} \boldsymbol{n}_{[1]} \\
& \nabla\left(\boldsymbol{t}_{[2]}^{\top} \nabla v^{Q}\right)=-\frac{3}{16\left|\Omega_{k}\right|}\left(\left(2 F_{[1]}-F_{[3]}\right) l_{[1]} \boldsymbol{n}_{[1]}+F_{[2]} l_{[2]} \boldsymbol{n}_{[2]}+F_{[3]} l_{[3]} \boldsymbol{n}_{[3]}\right) \\
& \nabla\left(\boldsymbol{t}_{[3]}^{\top} \nabla v^{Q}\right)=\frac{3}{16\left|\Omega_{k}\right|}\left(\left(2 F_{[1]}-F_{[2]}\right) l_{[1]} \boldsymbol{n}_{[1]}+F_{[2]} l_{[2]} \boldsymbol{n}_{[2]}+F_{[3]} l_{[3]} \boldsymbol{n}_{[3]}\right) .
\end{aligned}
$$

Introducing all these previous results in the equation for the divergence of $\mathbf{q}_{k}^{i C}$ (C.3), using the partition of unity property $\lambda_{[1]}+\lambda_{[2]}+\lambda_{[3]}=1$, and carefully simplifying all the terms yields

$$
\nabla \cdot \mathbf{q}_{k}^{i C}=-\phi_{i} F+\frac{1}{12}\left(2 F_{[1]}+F_{[2]}+F_{[3]}\right) .
$$

Finally joining equations (C.2) and (C.4) and using equation (27) yields

$$
\nabla \cdot\left(\boldsymbol{q}_{k}^{i}+\phi_{i} \boldsymbol{\nabla} u_{h}\right)=\nabla \cdot\left(\boldsymbol{q}_{k}^{i L}+\phi_{i} \boldsymbol{\nabla} u_{h}\right)+\nabla \cdot\left(\boldsymbol{q}_{k}^{i C}\right)=-\phi_{i} F+\nabla u_{h} \cdot \nabla \phi_{i}
$$

and therefore equation (C.1a) holds.

\section{Appendix D. Detailed expression for $\left\|q_{k}^{i}\right\|_{k}^{2}$}

Expanding the expression for the norm of $\left\|\boldsymbol{q}_{k}^{i}\right\|_{k}^{2}$ yields

$$
\left\|\boldsymbol{q}_{k}^{i}\right\|_{k}^{2}=\left\|\boldsymbol{q}_{k}^{i L}+\boldsymbol{q}_{k}^{i C}\right\|_{k}^{2}=\left\|\boldsymbol{q}_{k}^{i L}\right\|_{k}^{2}+\left\|\boldsymbol{q}_{k}^{i C}\right\|_{k}^{2}+2\left(\boldsymbol{q}_{k}^{i L}, \boldsymbol{q}_{k}^{i C}\right)_{k}
$$


where $(\cdot, \cdot)_{k}$ represents the $\mathcal{L}^{2}\left(\Omega_{k}\right)$ scalar product. Simple but thorough computations allow rewriting the three terms of the previous expression as

$$
\begin{aligned}
& \left\|\boldsymbol{q}_{k}^{i L}\right\|_{k}^{2}=\boldsymbol{\alpha}_{k}^{\top} \mathbf{M}_{k}^{L} \boldsymbol{\alpha}_{k}+\boldsymbol{\alpha}_{k}^{\top} \mathbf{b}_{k}^{L}+\mathrm{c}_{k}^{L}, \\
& \left(\boldsymbol{q}_{k}^{i L}, \boldsymbol{q}_{k}^{i C}\right)_{k}=\boldsymbol{\alpha}_{k}^{\top} \mathbf{b}_{k}^{L C}+\mathrm{c}_{k}^{L C} \\
& \left\|\boldsymbol{q}_{k}^{i C}\right\|_{k}^{2}=\mathrm{c}_{k}^{C}
\end{aligned}
$$

where

$$
\begin{aligned}
& \mathbf{M}_{k}^{L}=\frac{1}{24\left|\Omega_{k}\right|}\left(\begin{array}{cccc}
l_{[2]}^{2} l_{[3]}^{2} & -\frac{1}{2} l_{[2]}^{2} \boldsymbol{t}_{[1]}^{\top} \boldsymbol{t}_{[3]} & -l_{[2]} l_{[3]} \sigma_{k}^{\gamma_{[2]}} \sigma_{k}^{\gamma_{[3}} \boldsymbol{t}_{[2]}^{\top} \boldsymbol{t}_{[3]} & \frac{1}{2} l_{[2]} l_{[3]} \sigma_{k}^{\gamma_{[1]}} \sigma_{k}^{\gamma_{[3}} \boldsymbol{t}_{[1]}^{\top} \boldsymbol{t}_{[3]} \\
& l_{[1]}^{2} l_{[2]}^{2} & \frac{1}{2} l_{[2]} l_{[3]} \sigma_{k}^{\gamma_{[2]}} \sigma_{k}^{\gamma_{[3}} \boldsymbol{t}_{[1]}^{\top} \boldsymbol{t}_{[2]} & -\frac{1}{2} l_{[1]}^{2} l_{[2]} l_{[3]} \sigma_{k}^{\gamma_{[2]}} \sigma_{k}^{\gamma_{[3]}} \\
\operatorname{sym} & & l_{[2]}^{2} l_{[3]}^{2} & -\frac{1}{2} l_{[3]}^{2} \boldsymbol{t}_{[1]}^{\top} \boldsymbol{t}_{[2]} \\
& & & l_{[1]}^{2} l_{[3]}^{2}
\end{array}\right), \\
& \mathbf{b}_{k}^{L}=\frac{1}{24\left|\Omega_{k}\right|}\left(\begin{array}{c}
2 l_{[2]} l_{[3]} \sigma_{k}^{\gamma_{[2]}}\left(\nabla u_{h} \cdot \boldsymbol{n}_{[3]}\right) \boldsymbol{t}_{[2]}^{\top} \boldsymbol{t}_{[3]}-2 l_{[2]}^{2} l_{[3]}^{2} \sigma_{k}^{\gamma_{2]}}\left(\boldsymbol{\nabla} u_{h} \cdot \boldsymbol{n}_{[2]}\right) \\
-l_{[2]} l_{[3]} \sigma_{k}^{\gamma_{[2]}}\left(\boldsymbol{\nabla} u_{h} \cdot \boldsymbol{n}_{[3]}\right) \boldsymbol{t}_{[1]}^{\top} \boldsymbol{t}_{[2]}+l_{[2]}^{2} \sigma_{k}^{\gamma_{[2]}}\left(\boldsymbol{\nabla} u_{h} \cdot \boldsymbol{n}_{[2]}\right) \boldsymbol{t}_{[1]}^{\top} \boldsymbol{t}_{[3]} \\
-2 l_{[2]}^{2} l_{[3]}^{2} \sigma_{k}^{\gamma_{[3]}}\left(\boldsymbol{\nabla} u_{h} \cdot \boldsymbol{n}_{[3]}\right)+2 l_{[2]} l_{[3]} \sigma_{k}^{\gamma_{[3]}}\left(\boldsymbol{\nabla} u_{h} \cdot \boldsymbol{n}_{[2]}\right) \boldsymbol{t}_{[2]}^{\top} \boldsymbol{t}_{[3]} \\
l_{[3]}^{2} \sigma_{k}^{\gamma_{33}}\left(\boldsymbol{\nabla} u_{h} \cdot \boldsymbol{n}_{[3]}\right) \boldsymbol{t}_{[1]}^{\top} \boldsymbol{t}_{[2]}-l_{[2]} l_{[3]} \sigma_{k}^{\gamma_{33}}\left(\boldsymbol{\nabla} u_{h} \cdot \boldsymbol{n}_{[2]}\right) \boldsymbol{t}_{[1]}^{\top} \boldsymbol{t}_{[3]}
\end{array}\right) \\
& c_{k}^{L}=\frac{1}{24\left|\Omega_{k}\right|}\left(l_{[2]}^{2} l_{[3]}^{2}\left(\nabla u_{h} \cdot \boldsymbol{n}_{[3]}\right)^{2}+l_{[2]}^{2} l_{[3]}^{2}\left(\nabla u_{h} \cdot \boldsymbol{n}_{[2]}\right)^{2}-2 l_{[2]} l_{[3]}\left(\nabla u_{h} \cdot \boldsymbol{n}_{[2]}\right)\left(\boldsymbol{\nabla} u_{h} \cdot \boldsymbol{n}_{[3]}\right) \boldsymbol{t}_{[2]}^{\top} \boldsymbol{t}_{[3]}\right), \\
& \mathbf{b}_{k}^{L C}=\frac{1}{2880}\left(\begin{array}{c}
-14 F_{[1]} l_{[2]}\left(\boldsymbol{t}_{[2]}^{\top} \boldsymbol{t}_{[3]}-l_{[3]}^{2}\right)-F_{[2]} l_{[2]}\left(3 \boldsymbol{t}_{[2]}^{\top} \boldsymbol{t}_{[3]}-2 \boldsymbol{t}_{[1]}^{\top} \boldsymbol{t}_{[3]}+l_{[3]}^{2}\right)-F_{[3]} l_{[2]}\left(-3 l_{[3]}^{2}+2 \boldsymbol{t}_{[1]}^{\top} \boldsymbol{t}_{[3]}-\boldsymbol{t}_{[2]}^{\top} \boldsymbol{t}_{[3]}\right) \\
-6 F_{[1]} l_{[2]}\left(\boldsymbol{t}_{[1]}^{\top} \boldsymbol{t}_{[3]}-2 \boldsymbol{t}_{[1]}^{\top} \boldsymbol{t}_{[2]}\right)-3 F_{[2]} l_{[2]}\left(l_{[1]}^{2}-\boldsymbol{t}_{[2]}^{\top} \boldsymbol{t}_{[1]}\right)-F_{[3]} l_{[2]}\left(-\boldsymbol{t}_{[2]}^{\top} \boldsymbol{t}_{[1]}+2 \boldsymbol{t}_{[3]}^{\top} \boldsymbol{t}_{[1]}-3 l_{[1]}^{2}\right) \\
-14 F_{[1]} l_{[3]}\left(\boldsymbol{t}_{[2]}^{\top} \boldsymbol{t}_{[3]}-l_{[2]}^{2}\right)-F_{[2]} l_{[3]}\left(2 \boldsymbol{t}_{[1]}^{\top} \boldsymbol{t}_{[2]}-\boldsymbol{t}_{[2]}^{\top} \boldsymbol{t}_{[3]}-3 l_{[2]}^{2}\right)-F_{[3]} l_{[3]}\left(3 \boldsymbol{t}_{[2]}^{\top} \boldsymbol{t}_{[3]}-2 \boldsymbol{t}_{[1]}^{\top} \boldsymbol{t}_{[2]}+l_{[2]}^{2}\right) \\
-6 F_{[1]} l_{[3]}\left(\boldsymbol{t}_{[1]}^{\top} \boldsymbol{t}_{[2]}-2 \boldsymbol{t}_{[1]}^{\top} \boldsymbol{t}_{[3]}\right)-F_{[2]} l_{[3]}\left(2 \boldsymbol{t}_{[1]}^{\top} \boldsymbol{t}_{[2]}-\boldsymbol{t}_{[1]}^{\top} \boldsymbol{t}_{[3]}-3 l_{[1]}^{2}\right)-3 F_{[3]} l_{[3]}\left(-\boldsymbol{t}_{[1]}^{\top} \boldsymbol{t}_{[3]}+l_{[1]}^{2}\right)
\end{array}\right), \\
& \mathrm{c}_{k}^{L C}=\frac{1}{2880}\left(14 F_{[1]}\left(l_{[3]}\left(\boldsymbol{n}_{[3]} \cdot \nabla u_{h}\right)\left(\boldsymbol{t}_{[2]}^{\top} \boldsymbol{t}_{[3]}-l_{[2]}^{2}\right)+l_{[2]}\left(\boldsymbol{n}_{[2]} \cdot \nabla u_{h}\right)\left(\boldsymbol{t}_{[2]}^{\top} \boldsymbol{t}_{[3]}-l_{[3]}^{2}\right)\right)\right. \\
& +F_{[2]}\left(l_{[3]}\left(\boldsymbol{n}_{[3]} \cdot \nabla u_{h}\right)\left(2 \boldsymbol{t}_{[1]}^{\top} \boldsymbol{t}_{[2]}-\boldsymbol{t}_{[2]}^{\top} \boldsymbol{t}_{[3]}-3 l_{[2]}^{2}\right)+l_{[2]}\left(\boldsymbol{n}_{[2]} \cdot \nabla u_{h}\right)\left(-2 \boldsymbol{t}_{[1]}^{\top} \boldsymbol{t}_{[3]}+l_{[3]}^{2}+3 \boldsymbol{t}_{[2]}^{\top} \boldsymbol{t}_{[3]}\right)\right) \\
& \left.+F_{[3]}\left(l_{[2]}\left(\boldsymbol{n}_{[2]} \cdot \nabla u_{h}\right)\left(2 \boldsymbol{t}_{[1]}^{\top} \boldsymbol{t}_{[3]}-\boldsymbol{t}_{[2]}^{\top} \boldsymbol{t}_{[3]}-3 l_{[3]}^{2}\right)+l_{[3]}\left(\boldsymbol{n}_{[3]} \cdot \nabla u_{h}\right)\left(-2 \boldsymbol{t}_{[1]}^{\top} \boldsymbol{t}_{[2]}+l_{[2]}^{2}+3 \boldsymbol{t}_{[2]}^{\top} \boldsymbol{t}_{[3]}\right)\right)\right)
\end{aligned}
$$

and

$$
\begin{aligned}
c_{k}^{C}= & \frac{\left|\Omega_{k}\right|}{1451520}\left(61 l_{[1]}^{2}\left(F_{[2]}-F_{[3]}\right)^{2}\right. \\
& +\left(1240 F_{[1]}^{2}+518 F_{[1]} F_{[2]}-54 F_{[1]} F_{[3]}+61 F_{[2]}^{2}+27 F_{[3]}^{2}\right) l_{[2]}^{2} \\
& +\left(1240 F_{[1]}^{2}-54 F_{[1]} F_{[2]}+518 F_{[1]} F_{[3]}+27 F_{[2]}^{2}+61 F_{[3]}^{2}\right) l_{[3]}^{2} \\
& +2\left(F_{[2]}-F_{[3]}\right)\left(6 F_{[2]}+155 F_{[1]}+47 F_{[3]}\right) \boldsymbol{t}_{[1]}^{\top} \boldsymbol{t}_{[3]} \\
& -2\left(F_{[2]}-F_{[3]}\right)\left(47 F_{[2]}+155 F_{[1]}+6 F_{[3]}\right) \boldsymbol{t}_{[1]}^{\top} \boldsymbol{t}_{[2]} \\
& \left.+4\left(-310 F_{[1]}^{2}-58 F_{[1]} F_{[2]}-58 F_{[1]} F_{[3]}+3 F_{[2]}^{2}-28 F_{[2]} F_{[3]}+3 F_{[3]}^{2}\right) \boldsymbol{t}_{[2]}^{\top} \boldsymbol{t}_{[3]}\right) .
\end{aligned}
$$

Finally, joining equations (D.1) and yields the desired expression for $\left\|\boldsymbol{q}_{k}^{i}\right\|_{k}^{2}$ given in equation (28). 


\section{Appendix E. A novel construction of inter-element quadratic equilibrated fluxes}

The same approach introduced in section 5 can be considered to obtain a set of quadratic equilibrated tractions. In this case, the approximate tractions in the edges are quadratic functions in the edges. That is, if $\gamma$ is the edge joining nodes $m$ and $m^{\prime}$ respectively, then

$$
g_{\gamma}^{q}=\alpha_{\gamma}^{m} \lambda_{m}^{q}+\alpha_{\gamma}^{m m^{\prime}} \lambda_{m m^{\prime}}^{q}+\alpha_{\gamma}^{m^{\prime}} \lambda_{m^{\prime}}^{q}
$$

where $\lambda_{m}^{q}, \lambda_{m m^{\prime}}^{q}$ and $\lambda_{m^{\prime}}^{q}$ are the one-dimensional quadratic shape functions associated to the nodes $\boldsymbol{x}_{m}$ , $\boldsymbol{x}_{m m^{\prime}}$ and $\boldsymbol{x}_{m^{\prime}}$ respectively, where $\boldsymbol{x}_{m}$ and $\boldsymbol{x}_{m^{\prime}}$ are the two ends of edge $\gamma$ and $\boldsymbol{x}_{m m^{\prime}}=\left(\boldsymbol{x}_{m}+\boldsymbol{x}_{m^{\prime}}\right) / 2$ is its mid-point.

As in section 5, the equilibrated tractions are constructed using a subdomain-based technique. That is, for each star, we compute a set of weighted tractions $\left\{g_{\gamma}^{q i}\right\}_{\gamma \subset \omega_{i}}$ verifying both (17a), the equivalent of (17b) for $\bar{q}=1$, namely $g_{\gamma}^{i}=\phi_{i} \Pi_{\gamma}^{1} g_{\mathrm{N}}$ on $\gamma \subset \Gamma_{i} \cap \Gamma_{\mathrm{N}}$ and (20). These weighted tractions $g_{\gamma}^{q i}$ are defined using the weighted coefficients

$$
g_{\gamma}^{q i}=\alpha_{\gamma i}^{m} \lambda_{m}^{q}+\alpha_{\gamma i}^{m m^{\prime}} \lambda_{m m^{\prime}}^{q}+\alpha_{\gamma i}^{m^{\prime}} \lambda_{m^{\prime}}^{q}
$$

and the global tractions are recovered adding all the weighted contributions, namely $g_{\gamma}^{q}=\sum_{i=1}^{n_{\mathrm{np}}} g_{\gamma}^{q i}$. A unique set of effective tractions are computed solving a local constrained quadratic optimization problem. This problem, is obtained associating the tractions $g_{\gamma}^{q i}$ with a set of fluxes in the star. For each star $\omega_{i}$, consider the associated set of local equilibrated fluxes $\left\{\boldsymbol{q}_{k}^{q i}\right\}_{\Omega_{k} \subset \omega_{i}}$ such that

$$
\begin{aligned}
-\nabla \cdot\left(\boldsymbol{q}_{k}^{q i}+\phi_{i} \boldsymbol{\nabla} u_{h}\right) & =\phi_{i}\left(\Pi_{k}^{1} f-\kappa^{2} u_{h}\right)-\nabla u_{h} \cdot \nabla \phi_{i} & & \text { in } \Omega_{k} \\
\left(\boldsymbol{q}_{k}^{q i}+\phi_{i} \boldsymbol{\nabla} u_{h}\right) \cdot \boldsymbol{n}_{k}^{\gamma} & =\sigma_{k}^{\gamma} g_{\gamma}^{q i} & & \text { on } \gamma \subset \partial \Omega_{k},
\end{aligned}
$$

and find the tractions $g_{\gamma}^{q i}$ minimizing the norm of the local equilibrated fluxes, that is: find $g_{\gamma}^{q i}$ minimizing $\sum_{\Omega_{k} \subset \omega_{i}}\left\|\boldsymbol{q}_{k}^{q i}\right\|_{k}^{2}$.

The crucial point is that a closed formula for the local fluxes $\boldsymbol{q}_{k}^{q i}$ also exists for the quadratic fluxes, which allows rewriting the sum of the squared norms as an explicit quadratic function depending only on the tractions $g_{\gamma}^{q i}$.

To introduce the closed formula for the local fluxes, the notations introduced in Figures 1 and E.14 are used.

Assuming that the node $\boldsymbol{x}_{[1]}$ coincides with the central node of the star $\boldsymbol{x}_{i}$ so that $\lambda_{[1]}=\phi_{i}$, the local equilibrated fluxes $\boldsymbol{q}_{k}^{q i}$ verifying (E.1) are computed using three different contributions:

$$
\boldsymbol{q}_{k}^{q i}=\boldsymbol{q}_{k}^{q i Q}+\boldsymbol{q}_{k}^{q i C}+\boldsymbol{q}_{k}^{q i \nabla}
$$

The contribution due to the boundary conditions is a quadratic flux given by

$$
\mathbf{q}_{k}^{q i Q}=\frac{1}{2\left|\Omega_{k}\right|}\left(\boldsymbol{\rho}_{[1]}^{k} \lambda_{[1]}^{q}+\boldsymbol{\rho}_{[2]}^{k} \lambda_{[2]}^{q}+\boldsymbol{\rho}_{[3]}^{k} \lambda_{[3]}^{q}+\boldsymbol{\rho}_{[12]}^{k} \lambda_{[12]}^{q}+\boldsymbol{\rho}_{[23]}^{k} \lambda_{[23]}^{q}+\boldsymbol{\rho}_{[31]}^{k} \lambda_{[31]}^{q}\right),
$$




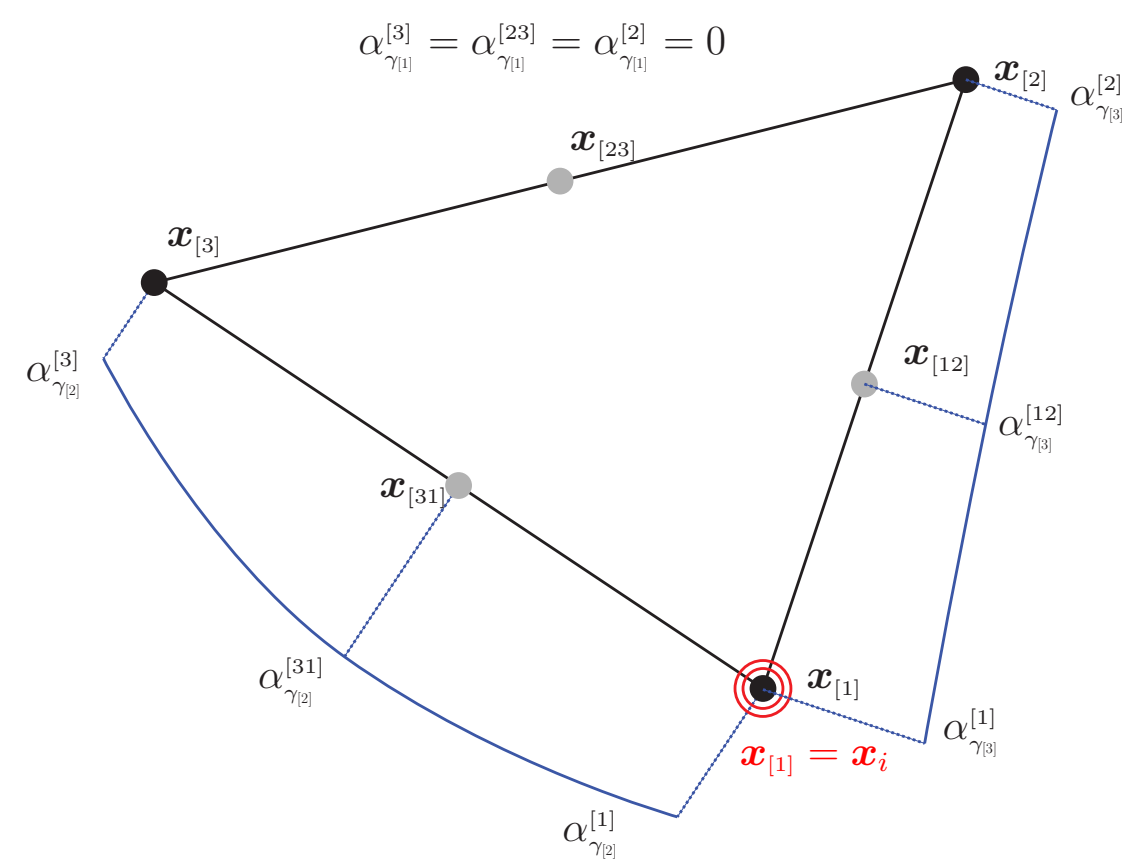

Figure E.14: Notation for the vertices and edges of an element contained in star $\omega_{i}$.

for

$$
\begin{aligned}
& \boldsymbol{\rho}_{[1]}^{k}=\left.l_{[3]}\left(\sigma_{k}^{\gamma} g_{\gamma}^{q i}-\phi_{i} \boldsymbol{\nabla} u_{h} \cdot \boldsymbol{n}_{k}\right)\right|_{\gamma_{[3]}}\left(\boldsymbol{x}_{[1]}\right) \boldsymbol{t}_{[2]}-\left.l_{[2]}\left(\sigma_{k}^{\gamma} g_{\gamma}^{q i}-\phi_{i} \boldsymbol{\nabla} u_{h} \cdot \boldsymbol{n}_{k}\right)\right|_{\gamma_{[2]}}\left(\boldsymbol{x}_{[1]}\right) \boldsymbol{t}_{[3]}, \\
& \boldsymbol{\rho}_{[2]}^{k}=\left.l_{[1]}\left(\sigma_{k}^{\gamma} g_{\gamma}^{q i}-\phi_{i} \boldsymbol{\nabla} u_{h} \cdot \boldsymbol{n}_{k}\right)\right|_{\gamma_{[1]}}\left(\boldsymbol{x}_{[2]}\right) \boldsymbol{t}_{[3]}-\left.l_{[3]}\left(\sigma_{k}^{\gamma} g_{\gamma}^{q i}-\phi_{i} \boldsymbol{\nabla} u_{h} \cdot \boldsymbol{n}_{k}\right)\right|_{\gamma_{[3]}}\left(\boldsymbol{x}_{[2]}\right) \boldsymbol{t}_{[1]}, \\
& \boldsymbol{\rho}_{[3]}^{k}=\left.l_{[2]}\left(\sigma_{k}^{\gamma} g_{\gamma}^{q i}-\phi_{i} \boldsymbol{\nabla} u_{h} \cdot \boldsymbol{n}_{k}\right)\right|_{\gamma_{[2]}}\left(\boldsymbol{x}_{[3]}\right) \boldsymbol{t}_{[1]}-\left.l_{[1]}\left(\sigma_{k}^{\gamma} g_{\gamma}^{q i}-\phi_{i} \boldsymbol{\nabla} u_{h} \cdot \boldsymbol{n}_{k}\right)\right|_{\gamma_{[1]}}\left(\boldsymbol{x}_{[3]}\right) \boldsymbol{t}_{[2]}, \\
& \boldsymbol{\rho}_{[12]}^{k}=\left.2\left|\Omega_{k}\right|\left(\sigma_{k}^{\gamma} g_{\gamma}^{q i}-\phi_{i} \boldsymbol{\nabla} u_{h} \cdot \boldsymbol{n}_{k}\right)\right|_{\gamma_{[3]}}\left(\boldsymbol{x}_{[12]}\right) \boldsymbol{n}_{[3]}, \\
& \boldsymbol{\rho}_{[23]}^{k}=\left.2\left|\Omega_{k}\right|\left(\sigma_{k}^{\gamma} g_{\gamma}^{q i}-\phi_{i} \boldsymbol{\nabla} u_{h} \cdot \boldsymbol{n}_{k}\right)\right|_{\gamma_{[1]}}\left(\boldsymbol{x}_{[23]}\right) \boldsymbol{n}_{[1]}, \\
& \boldsymbol{\rho}_{[31]}^{k}=\left.2\left|\Omega_{k}\right|\left(\sigma_{k}^{\gamma} g_{\gamma}^{q i}-\phi_{i} \boldsymbol{\nabla} u_{h} \cdot \boldsymbol{n}_{k}\right)\right|_{\gamma_{[2]}}\left(\boldsymbol{x}_{[31]}\right) \boldsymbol{n}_{[2]} .
\end{aligned}
$$

The cubic flux $\boldsymbol{q}_{k}^{q i C}$ that contributes to the divergence restriction is the same as the one associated to the linear fluxes, defined in (24), that is $\boldsymbol{q}_{k}^{q i C}=\boldsymbol{q}_{k}^{i C}$. Finally, an additional third term $\boldsymbol{q}_{k}^{q i \nabla}$ is introduced to compensate the divergence of the now quadratic boundary flux $\boldsymbol{q}_{k}^{q i Q}$. This flux is given by

$$
\mathbf{q}_{k}^{q i \nabla}=\frac{1}{3}\left(\beta_{[1]} \boldsymbol{t}_{[1]} \boldsymbol{t}_{[1]}^{\top}+\beta_{[2]} \boldsymbol{t}_{[2]} \boldsymbol{t}_{[2]}^{\top}+\beta_{[3]} \boldsymbol{t}_{[3]} \boldsymbol{t}_{[3]}^{\top}\right) v^{\nabla}
$$

where

$$
\begin{aligned}
v^{\nabla}=\frac{1}{2\left|\Omega_{k}\right|^{3}}( & l_{[1]}^{2} \boldsymbol{n}_{[1]} \boldsymbol{n}_{[1]}^{\top} \boldsymbol{\rho}_{[1]}^{k}+l_{[2]}^{2} \boldsymbol{n}_{[2]} \boldsymbol{n}_{[2]}^{\top} \boldsymbol{\rho}_{[2]}^{k}+l_{[3]}^{2} \boldsymbol{n}_{[3]} \boldsymbol{n}_{[3]}^{\top} \boldsymbol{\rho}_{[3]}^{k} \\
& +l_{[1]} l_{[2]}\left(\boldsymbol{n}_{[1]} \boldsymbol{n}_{[2]}^{\top}+\boldsymbol{n}_{[2]} \boldsymbol{n}_{[1]}^{\top}\right) \boldsymbol{\rho}_{[12]}^{k}+l_{[2]} l_{[3]}\left(\boldsymbol{n}_{[2]} \boldsymbol{n}_{[3]}^{\top}+\boldsymbol{n}_{[3]} \boldsymbol{n}_{[2]}^{\top}\right) \boldsymbol{\rho}_{[23]}^{k} \\
& \left.+l_{[3]} l_{[1]}\left(\boldsymbol{n}_{[3]} \boldsymbol{n}_{[1]}^{\top}+\boldsymbol{n}_{[1]} \boldsymbol{n}_{[3]}^{\top}\right) \boldsymbol{\rho}_{[31]}^{k}\right) .
\end{aligned}
$$

The weighted equilibration condition on each element of the star $\Omega_{k} \subset \omega_{i}$, equation (20), can be expressed as

$$
\begin{gathered}
l_{[2]} \sigma_{k}^{\gamma_{[2]}}\left(\alpha_{\gamma_{[2]}}^{[1]}+4 \alpha_{\gamma_{[2]}}^{[31]}+\alpha_{\gamma_{[2]}}^{[3]}\right)+l_{[3]} \sigma_{k}^{\gamma_{[3]}}\left(\alpha_{\gamma_{[3]}}^{[1]}+4 \alpha_{\gamma_{[3]}}^{[12]}+\alpha_{\gamma_{[3]}}^{[2]}\right) \\
=-\frac{\left|\Omega_{k}\right|}{2}\left(2 F_{[1]}+F_{[2]}+F_{[3]}-12 \nabla u_{h} \cdot \nabla \phi_{i}\right) .
\end{gathered}
$$

It is worth noting that, as in the linear traction case, this condition is both required to ensure that 
the local elementary problems are solvable, and to ensure that the flux $\boldsymbol{q}_{k}^{q i}$ verifies (E.1). Also note that, since $g_{\gamma_{[1]}}^{q i}=0$, the tractions in each element of the star are described using only six degrees of freedom,

$$
g_{\gamma_{[2]}}^{q i}=\alpha_{\gamma_{[2]}}^{[3]} \lambda_{[3]}^{q}+\alpha_{\gamma_{[2]}}^{[31]} \lambda_{[31]}^{q}+\alpha_{\gamma_{[2]}}^{[1]} \lambda_{[1]}^{q} \quad, \quad g_{\gamma_{[3]}}^{q i}=\alpha_{\gamma_{[3]}}^{[1]} \lambda_{[1]}^{q}+\alpha_{\gamma_{[3]}}^{[12]} \lambda_{[12]}^{q}+\alpha_{\gamma_{[3]}}^{[2]} \lambda_{[2]}^{q}
$$

where as in the linear case, the subscript $i$ referring to the star is omitted in the tractions coefficients, see Figure E.14.

[1] M. Ainsworth, J. T. Oden, A posteriori error estimation in finite element analysis, Pure and Applied Mathematics (New York), Wiley-Interscience [John Wiley \& Sons], New York, 2000. URL http://dx.doi.org/10.1002/9781118032824

[2] M. Ainsworth, T. Vejchodský, Fully computable robust a posteriori error bounds for singularly perturbed reaction-diffusion problems, Numer. Math. 119 (2) (2011) 219-243. URL http://dx.doi.org/10.1007/s00211-011-0384-1

[3] M. Ainsworth, T. Vejchodský, Robust error bounds for finite element approximation of reactiondiffusion problems with non-constant reaction coefficient in arbitrary space dimension, Comput. Methods Appl. Mech. Engrg. 281 (2014) 184-199.

URL http://dx.doi.org/10.1016/j.cma.2014.08.005

[4] C. Carstensen, R. H. W. Hoppe, Convergence analysis of an adaptive nonconforming finite element method, Numer. Math. 103 (2) (2006) 251-266.

[5] L. Chamoin, P. Díez (eds.), Verifying calculations - forty years on, Springer Briefs in Applied Sciences and Technology, Springer, Cham, 2016, An overview of classical verification techniques for FEM simulations.

URL http://dx.doi.org/10.1007/978-3-319-20553-3

[6] R. Cottereau, L. Chamoin, P. Díez, Strict error bounds for linear and nonlinear solid mechanics problems using a patch-based flux-free method, Mécanique \& Industries 11 (3-4) (2010) 249-254. URL http://dx.doi.org/10.1051/meca/2010049

[7] R. Cottereau, P. Díez, A. Huerta, Strict error bounds for linear solid mechanics problems using a subdomain-based flux-free method, Comput. Mech. 44 (4) (2009) 533-547.

URL http://dx.doi.org/10.1007/s00466-009-0388-1

[8] W. Dörfler, A convergent adaptive algorithm for Poisson's equation, SIAM J. Numer. Anal. 33 (3) (1996) 1106-1124.

URL http://dx.doi.org/10.1137/0733054

[9] P. Dez, N. Pars, A. Huerta, Error Estimation and Quality Control, vol. 3(15), chap. 144, John Wiley \& Sons, New York, 2010, pp. 1725-1734.

URL http://dx.doi.org/10.1002/9780470686652. eae164

[10] P. Ladevèze, L. Chamoin, É. Florentin, A new non-intrusive technique for the construction of admissible stress fields in model verification, Comput. Methods Appl. Mech. Engrg. 199 (9-12) (2010) 766-777.

URL http://dx.doi.org/10.1016/j.cma.2009.11.007

[11] P. Ladevèze, D. Leguillon, Error estimate procedure in the finite element method and applications, SIAM J. Numer. Anal. 20 (3) (1983) 485-509. 
[12] P. Ladevèze, P. Rougeot, New advances on a posteriori error on constitutive relation in f.e. analysis, Comput. Methods Appl. Mech. Engrg. 150 (1-4) (1997) 239-249, symposium on Advances in Computational Mechanics, Vol. 2 (Austin, TX, 1997).

URL http://dx.doi .org/10.1016/S0045-7825(97)00089-3

[13] J. T. Oden, Y. Feng, Local and pollution error estimation for finite element approximations of elliptic boundary value problems, J. Comput. Appl. Math. 74 (1-2) (1996) 245-293, TICAM Symposium (Austin, TX, 1995).

[14] N. Parés, J. Bonet, A. Huerta, J. Peraire, The computation of bounds for linear-functional outputs of weak solutions to the two-dimensional elasticity equations, Comput. Methods Appl. Mech. Engrg. 195 (4-6) (2006) 406-429.

[15] N. Parés, P. Díez, A. Huerta, Subdomain-based flux-free a posteriori error estimators, Comput. Methods Appl. Mech. Engrg. 195 (4-6) (2006) 297-323.

URL http://dx.doi.org/10.1016/j.cma.2004.06.047

[16] N. Parés, P. Díez, A. Huerta, Bounds of functional outputs for parabolic problems. Part I: Exact bounds of the discontinuous galerkin time discretization, Comput. Methods Appl. Mech. Engrg. 197 (19-20) (2008) 1641-1660.

[17] N. Parés, P. Díez, A. Huerta, Bounds of functional outputs for parabolic problems.

Part II: Bounds of the exact solution, Comput. Methods Appl. Mech. Engrg. 197 (19-20) (2008) 1661-1679.

[18] N. Parés, P. Díez, A. Huerta, Exact bounds for linear outputs of the advection-diffusion-reaction equation using flux-free error estimates, SIAM J. Sci. Comput. 31 (4) (2009) 3064-3089.

URL http://dx.doi.org/10.1137/080724356

[19] N. Parés, P. Díez, A. Huerta, Computable exact bounds for linear outputs from stabilized solutions of the advection-diffusion-reaction equation, Internat. J. Numer. Methods Engrg. 93 (5) (2013) 483-509.

URL http://dx.doi.org/10.1002/nme.4396

[20] N. Parés, H. Santos, P. Díez, Guaranteed energy error bounds for the Poisson equation using a flux-free approach: solving the local problems in subdomains, Internat. J. Numer. Methods Engrg. 79 (10) (2009) 1203-1244.

URL http://dx.doi.org/10.1002/nme.2593

[21] F. Pled, L. Chamoin, P. Ladevèze, An enhanced method with local energy minimization for the robust a posteriori construction of equilibrated stress fields in finite element analyses, Comput. Mech. 49 (3) (2012) 357-378.

URL http://dx.doi.org/10.1007/s00466-011-0645-y

[22] S. Repin, S. Sauter, A. Smolianski, A posteriori error estimation for the Poisson equation with mixed Dirichlet/Neumann boundary conditions, J. Comput. Appl. Math. 164/165 (2004) 601612, Proceedings of the 10th International Congress on Computational and Applied Mathematics (ICCAM-2002).

URL http://dx.doi.org/10.1016/S0377-0427(03)00491-6

[23] V. Rey, P. Gosselet, C. Rey, Study of the strong prolongation equation for the construction of statically admissible stress fields: implementation and optimization, Comput. Methods Appl. Mech. Engrg. 268 (2014) 82-104.

URL http://dx.doi.org/10.1016/j.cma.2013.08.021 
[24] A. M. Sauer-Budge, J. Bonet, A. Huerta, J. Peraire, Computing bounds for linear functionals of exact weak solutions to Poisson's equation, SIAM J. Numer. Anal. 42 (4) (2004) 1610-1630 (electronic).

[25] A. M. Sauer-Budge, J. Peraire, Computing bounds for linear functionals of exact weak solutions to the advection-diffusion-reaction equation, SIAM J. Sci. Comput. 26 (2) (2004) 636-652. URL http://dx.doi.org/10.1137/S1064827503427121

[26] T. Vejchodský, Guaranteed and locally computable a posteriori error estimate, IMA J. Numer. Anal. 26 (3) (2006) 525-540.

[27] Z. C. Xuan, N. Parés, J. Peraire, Computing upper and lower bounds for the $J$-integral in twodimensional linear elasticity, Comput. Methods Appl. Mech. Engrg. 195 (4-6) (2006) 430-443. 